\title{
Transferability of Data Related to the Underground Test Area Project, \\ Nevada Test Site, \\ Nye County, Nevada
}

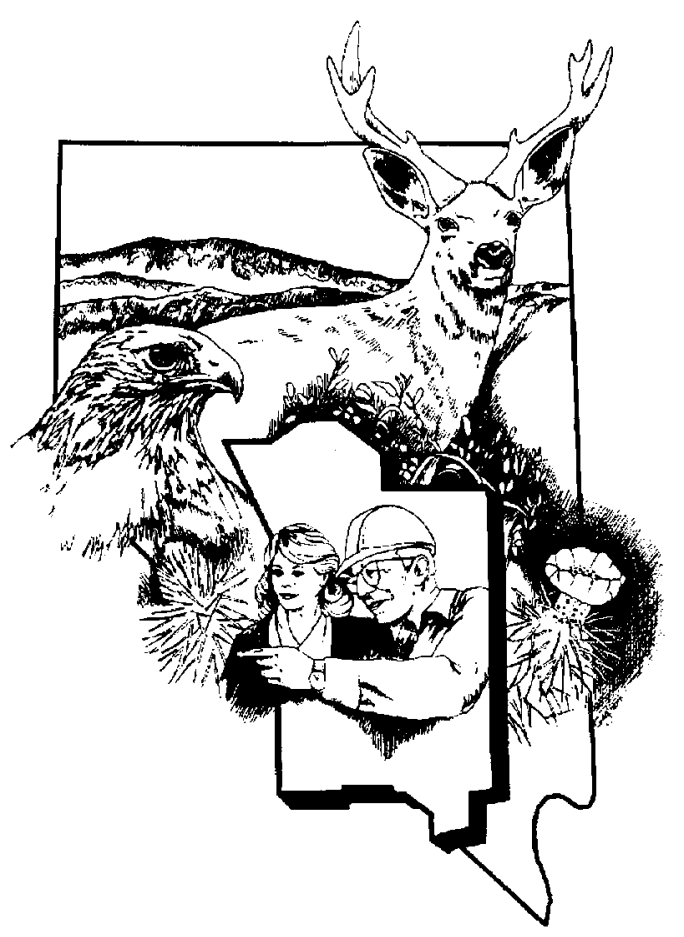

\section{Revision No.: 0}

June 2004

Prepared for U.S. Department of Energy under Contract No. DE-AC52-03NA99205.

Approved for public release; further dissemination unlimited. 
Available for public sale, in paper, from:

U.S. Department of Commerce

National Technical Information Service

5285 Port Royal Road

Springfield, VA 22161

Phone: 800.553 .6847

Fax: 703.605.6900

Email: orders@ntis.gov

Online ordering: http://www.ntis.gov/ordering.htm

Available electronically at $\underline{h t t p: / / w w w . o s t i . g o v / b r i d g e ~}$

Available for a processing fee to U.S. Department of Energy and its contractors, in paper, from:

U.S. Department of Energy

Office of Scientific and Technical Information

P.O. Box 62

Oak Ridge, TN 37831-0062

Phone: 865.576 .8401

Fax: 865.576.5728

Email: reports@adonis.osti.gov

Reference herein to any specific commercial product, process, or service by trade name, trademark, manufacturer, or otherwise, does not necessarily constitute or imply its endorsement, recommendation, or favoring by the United States Government or any agency thereof or its contractors or subcontractors. 


\section{TRANSFERABILITY OF DATA RELATED TO THE UNDERGROUND TEST AREA PROJECT, NEVADA TEST SITE, NYE COUNTY, NEVADA}

Contributors:

Richard Waddell, GeoTrans Inc.

Paul Reimus, Los Alamos National Laboratory (LANL)

Craig Shirley, Desert Research Institute (DRI)

Technical Reviewers:

Ward Hawkins, LANL

Gayle Pawloski, Lawrence Livermore National Laboratory (LLNL)

Jeff Daniels, LLNL

Randy Laczniak, U.S. Geological Survey (USGS)

Tim Rose, LLNL

Ron Hershey, DRI

Reed Maxwell, LLNL

Chris Benedict, GeoTrans, Inc.

Ahmed Hassan, DRI

Steve Carle, LLNL

Derek Sloop, Shaw E\&I

M.J. Umari, USGS

Chuck Russell, DRI

Revision No.: 0

June 2004

Stoller-Navarro Joint Venture 7710 W. Cheyenne, Building 3

Las Vegas, NV 89129 
TRANSFERABILITY OF DATA RELATED TO THE

UNDERGROUND TEST AREA PROJECT, NEVADA TEST SITE, NYE COUNTY, NEVADA 


\section{Table of Contents}

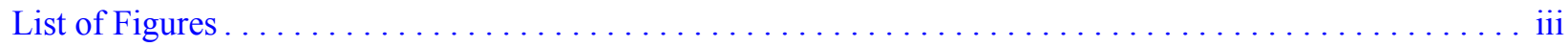

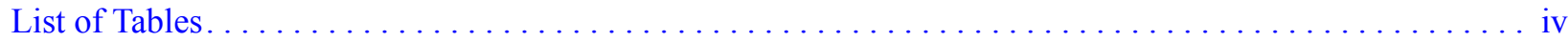

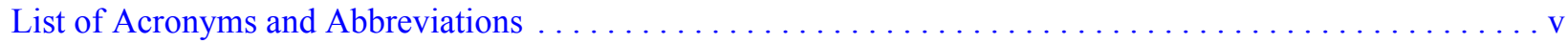

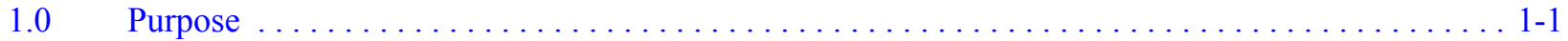

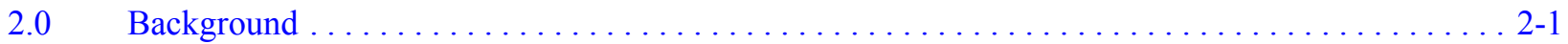

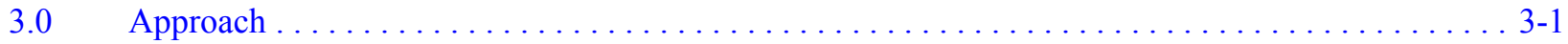

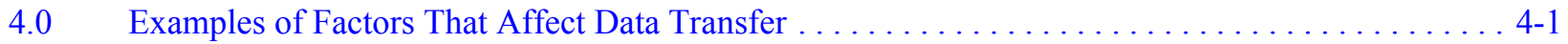

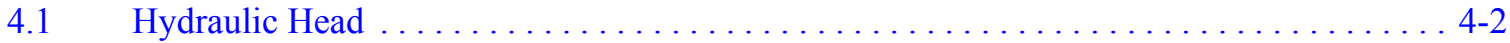

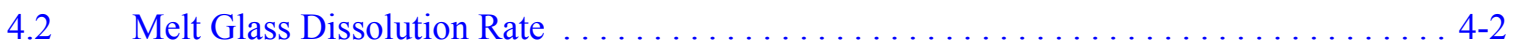

4.3 Bulk Hydraulic Conductivity . . . . . . . . . . . . . . . . $\ldots \ldots \ldots \ldots \ldots$

4.4 Effective Porosity. . . . . . . . . . . . . . . . . . . . . . . . . . . . .

4.5 Sorptive Behavior of Radionuclides . . . . . . . . . . . . . . . . . . . . 4-4

5.0 Recommended Steps for Establishing the Data Transfer Protocol for a Parameter . . . . . . . . . . . 5-1

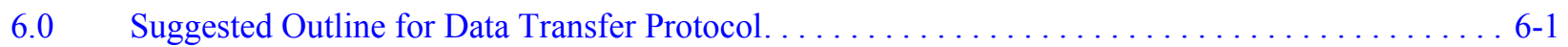

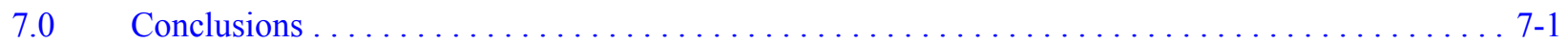

$8.0 \quad$ References . . . . . . . . . . . . . . . . . . . . . . . . . . . . . . . . . . . $8-1$

\section{Appendix A - Establishing the Similarity of Geologic Settings: A Formal Statistical Approach}

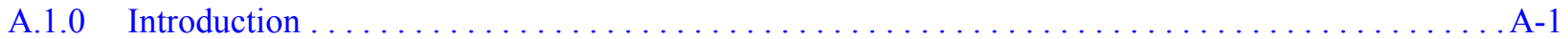

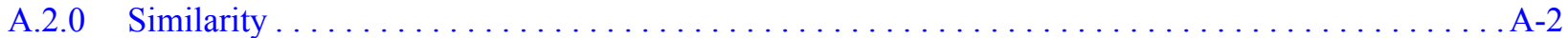

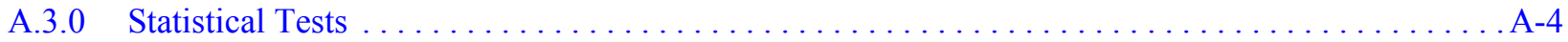

A.4.0 Spatial Statistics. . . . . . . . . . . . .

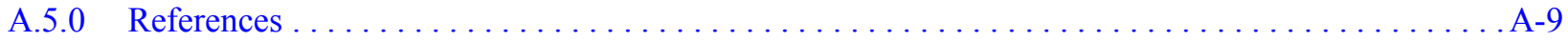

\section{Appendix B - Recommended Approach for Determining Emphasis Given to Transferred Data}

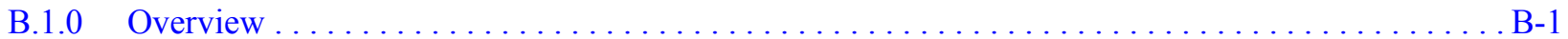

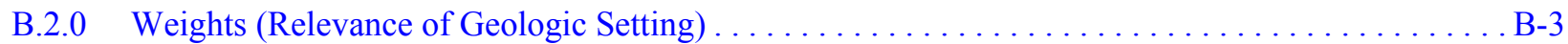

B.3.0 Multipliers (Measurement Method and Quality of Analyses and Documentation) . . . . . . . . . B-5

B.4.0 Overall Scores . . . . . . . . . . . . . . . . . . . . . . . . . . . . . . . . . . . . . . . . . . . . . . . B-7

B.5.0 Documentation. . . . . . . . . . . . . . . . . . . . . . . . . . . . . . . . . . . B 


\section{Table of Contents (Continued)}

B.6.0 Use of Weights and Multipliers in Downstream Analyses . . . . . . . . . . . . . . . . B-9 Attachments - Parameter Descriptions

Attachment A - Property Description - Porosity

Attachment B - Property Description - Matrix Diffusion 


\section{List of Figures}

Number

Title

Page

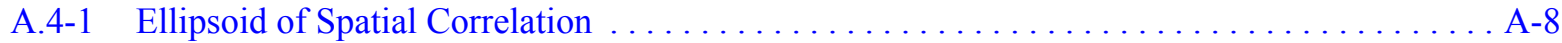

B.6-1 Cumulative Distribution Function Associated With the Data of Table B.6-1 . . . . . . . . B-11 


\section{List of Tables}

Number

Title

Page

B.6-1 Combined Weight and Multiplier Scores for Hypothetical Parameter Data . . . . . . . . . . B-10

B.6-2 Results of Manipulation of Data in Table B.6-1 to Obtain

Cumulative Distribution Function of Parameter Values. . . . . . . . . . . . . . . . . . . . . B-11 


\section{List of Acronyms and Abbreviations}

$\mathrm{AC}$

CAU

$\mathrm{CDF}$

$\mathrm{CO}_{2}$

DRI

EPA

FFACO

FGE

GPR

HSU

$\mathrm{K}_{\mathrm{d}}$

K-S

LANL

LLNL

NMR

NRC

NTS

$\mathrm{QA} / \mathrm{QC}$

REV

$\mathrm{RF}$

TWG

UGTA

USGS

YMP

2-D
Alternating current

Corrective Action Unit

Cumulative distribution function

Carbon dioxide

Desert Research Institute

U.S. Environmental Protection Agency

Federal Facility Agreement and Consent Order

Forced Gradient Experiment

Ground Penetrating Radar

Hydrostratigraphic Unit

Distribution coefficient

Kolmogorov-Smironov

Los Alamos National Laboratory

Lawrence Livermore National Laboratory

Nuclear Magnetic Resonance

U.S. Nuclear Regulatory Commission

Nevada Test Site

Quality assurance and quality control

Representative elementary volume

Radio frequency

Technical Working Group

Underground Test Area

U.S. Geological Survey

Yucca Mountain Project

Two-dimensional 


\subsection{Purpose}

This document is the collaborative effort of the members of an ad hoc subcommittee of the Underground Test Area (UGTA) Technical Working Group (TWG). The principal authors include Richard Waddell, GeoTrans, Inc; Paul Reimus, Los Alamos National Laboratory (LANL); and Craig Shirley, Desert Research Institute (DRI). The technical reviewers supporting the development of this document include Ward Hawkins, LANL; Gayle Pawloski, Lawrence Livermore National Laboratory (LLNL); Jeff Daniels, LLNL; Randy Laczniak, U.S. Geological Survey (USGS); Tim Rose, LLNL; Ron Hershey, DRI; Reed Maxwell, LLNL; Chris Benedict, GeoTrans, Inc.; Ahmed Hassan, DRI; Steve Carle, LLNL; Derek Sloop, Shaw E\&I; M.J. Umari, USGS; and Chuck Russell, DRI. Each subcommittee member provided valuable insight into the transferability of data related to the UGTA. This document is a synthesis of these insights.

The UGTA Project relies on data from a variety of sources; therefore, a process is needed to identify relevant factors for determining whether material-property data collected from other areas can be used to support groundwater flow, radionuclide transport, and other models within a Corrective Action Unit (CAU), and for documenting the data transfer decision and process. This document describes the overall data transfer process. Separate Parameter Descriptions will be prepared that provide information for selected specific parameters as determined by the U.S. Department of Energy (DOE) UGTA Project Manager.

Two appendices support this document. Appendix A discusses a formal approach to assessing the similarity of geologic settings at different locations. Appendix B provides a weighting procedure that may be useful for determining the reliability of transferred data.

This document and its accompanying appendices do not provide the specific criteria to be used for transfer of data for specific uses. Rather, the criteria will be established by separate parameter-specific and model-specific Data Transfer Protocols. The CAU Data Documentation Packages and data analysis reports will apply the protocols and provide or reference a document with the data transfer evaluations and decisions. 


\subsection{Background}

The UGTA project is collecting and evaluating data for use in predicting the movement of radionuclides from the sites of underground nuclear tests. The prediction of the transport of radionuclides depends on knowledge of the properties of the groundwater system, and the cavity/chimney environment at the underground test locations. Predictions will be made using groundwater flow and transport models, which require that values for parameters be specified throughout the model domain.

Even for small sites, it is not feasible to fully characterize the groundwater system, and simplifications and assumptions are made to facilitate the decision-making process. Information on material properties from similar materials at different locations is commonly used to estimate the properties at a site. The term "data transfer" is used to indicate the use of data not collected at a specific location within the CAU. It is assumed the transferred data are representative of site conditions. During evaluations of contaminant transport at typical hazardous waste sites, the effects of this assumption are not evaluated through an uncertainty analysis. However, data (such as contaminant concentrations) are available at typical contaminated sites to guide model calibration, and modeling results can therefore be relied upon for the decision-making process. However, the UGTA CAUs are not typical and a more formalized process for data transfer is needed.

In the underground nuclear testing areas at the Nevada Test Site (NTS), a large amount of information has been collected on the geology and some material properties. Further, pertinent data have been collected in volcanic rocks for the Yucca Mountain Project (YMP). Still, the data sets can never be considered truly complete. It is neither technically nor financially feasible to produce such a "complete" data set. However, the use of data from other sites, when and where appropriate, can help fill in gaps in understanding at a given location, and can also be very helpful in reducing uncertainty in modeling predictions.

This document describes a procedure for determining whether data from other locations can be used by the UGTA project to predict the transport of radionuclides. When adequate data are available, statistical testing may be used to test whether two areas are sufficiently similar to directly use the data from outside the CAU; this is considered to be the most robust approach (Appendix A). This document also provides a procedure for determining how much emphasis or weight should be placed on accepted data relative to CAU-specific data or alternative data sets from other locations (Appendix B). This document is structured as follows: 
- Parameter Descriptions are a key part of this process. Parameter Descriptions provide detailed information on the definition and use of key parameters, including factors which affect the parameter values (such as lithology, alteration, depth of burial, and structural features), how the parameters are measured, the reliability and representativeness of different measurement techniques, and guidance on approaches for evaluating the representativeness of parameter measurements based on their reliability and other attributes. Attachments A and B provide guidance on the transferability of porosity data and matrix diffusion data. Other parameter descriptions will be prepared and supplemented, as needed, based on recommendations to, and concurrence by, the U.S. Department of Energy, National Nuclear Security Administration Nevada Site Office (NNSA/NSO) UGTA Project Manager. However, Parameter Descriptions are not required in order for data transfer to be performed; they are intended to provide guidance and information for the more important and complex parameters.

- Data Transfer Protocols will describe the detailed process and criteria for data transfer to be applied to individual parameters for specific uses (see Section 5.0 and Section 6.0). The actual justification for the use of the data will be documented in the data documentation packages specific to each CAU and/or application. 


\subsection{Approach}

An important component of the UGTA strategy is the explicit evaluation of the effects of uncertainty, so that scientists, managers, and other decision makers can be better informed. Therefore, the data-transfer process must consider whether additional uncertainty in parameter values are created by the transfer process, and provide estimates of the uncertainty. The data-transfer process and requirements presented here are based on the recognition that uncertainty exists, and the concept that resources should not be expended to reduce the uncertainty in parameter values, unless uncertainty in the modeling predictions is significantly decreased by the reduction of parameter uncertainty.

There are two different approaches considered for UGTA use. These approaches include:

\section{Reject Unless Accepted}

Under this approach, data from other areas would not be used unless there was an explicit demonstration that the data meet affirmative acceptance criteria (e.g., the data are from an environment that was characterized sufficiently and demonstrated to be similar to the CAU environment). Acceptance criteria would be set, such as the measurement technique must be appropriate, the geologic environment or geochemical environment must be similar, quality assurance and quality control (QA/QC) requirements must be met. The acceptance criteria could be set to reflect the sensitivity of the parameter value to the particular setting or the sensitivity of predictions to the parameter values. This approach is most appropriate for sites such as Yucca Mountain where restrictive U.S Nuclear Regulatory Commission (NRC) licensing standards will be used. A Yucca Mountain example might be the use of corrosion data for water chemistries similar (but not identical) to those at Yucca Mountain. In order for such data to be used, it would be necessary to show that the differences in water chemistry would have negligible (or predictable) differences in corrosion rates. Another example would be use of studies of natural analogues for predicting radionuclide transport at Yucca Mountain. Less restrictive acceptance criteria might be appropriate for UGTA use.

\section{Accept Unless Rejected}

The philosophy for this approach is that a measurement is valid for use, if it is not disqualified for use. Thus, it is necessary to set disqualification criteria (e.g., must not be an invalid measurement technique, must not be from a dissimilar geologic environment for characteristics that affect the use of data, must not be a location-sensitive measurement). Again, the disqualification criteria can be set to reflect the importance of the parameter and the sensitivity to the setting. 
The differences between the two approaches are subtle, but important. The first (reject unless accepted) implies a more restrictive approach, in which uncertainty must be minimized and in which the acceptance criteria are loosened only with justification. It also requires a stricter degree of proof. The second approach (accept unless rejected) implies that greater uncertainty in parameters may be acceptable, and that the disqualification criteria will be tightened, as necessary, to reduce uncertainty in the predictions. The second approach is consistent with the UGTA approach described in the Federal Facility Agreement and Consent Order (FFACO) (1996), in which uncertainty in the predictions is reduced as necessary through additional data collection and/or data interpretation. The UGTA modeling approach is designed to address the effects of uncertainty, and to determine whether decreasing uncertainty is warranted. If so, the program has the option of using more stringent criteria for accepting the data and going through the additional effort of meeting the criteria, or of collecting CAU-specific information.

Because the accept-unless-rejected approach is consistent with the FFACO approach, it is the approach adopted for this document. Thus, steps in the evaluation are:

1. Establish the disqualification criteria, based on the use of the parameter and its importance, as currently viewed. These criteria are established before the modeling simulations and uncertainty and parameter sensitivity evaluations are performed. Changes in the criteria might be expected as the CAU investigations and modeling progress through the FFACO process. Thus, if it is determined that more restrictive criteria are needed for a particular parameter, it will be necessary to repeat the transferability evaluation. If the previously used criteria are restrictive enough, it will not be necessary to re-perform the evaluation.

2. Evaluate whether the measurement technique used would disqualify the measurement for use by UGTA.

3. Evaluate whether the geologic, geochemical, hydrologic, or other factors would disqualify the measurement for use by UGTA.

4. Document the process and data used in sufficient detail that other workers can understand and repeat the process. This information should be incorporated either directly or by reference in a database.

5. As the FFACO process progresses from Phase I to Phase II, and perhaps to subsequent phases, this data transfer process should be repeated for the parameters that have the greatest effect on the modeling predictions. 


\subsection{Examples of Factors That Affect Data Transfer}

This section considers several different parameter types to explore and illustrate the data transfer process. These parameters are discussed only to provide examples, not to be prescriptive for these or other parameters. They were selected to present a range of parameters and associated factors, and this discussion is not intended to imply that Parameter Descriptions should be developed for them. As indicated above, the data transfer disqualification criteria may need to be adjusted if the uncertainty in the parameter is a significant source of uncertainty in the simulation results.

The approach for implementing the data transfer process for models to be used to predict radionuclide transport needs to be parameter specific. There may be different considerations, resulting from:

- Parameter characteristics, including underlying dependencies on material properties or other parameters

- Similarity of donor and receptor hydrostratigraphic units (HSUs), with respect to geologic setting and other relevant characteristics for the parameter of concern

- Type of measurement and/or interpretative technique, including measurement scale

- Quality of documentation of measurements and interpretive methods

- Modeling approach, including conceptual models and model scale

- Heterogeneity

- $\quad$ Range in values

- Sensitivity of contaminant boundary to parameter value

Development of an appropriate approach for data transfer requires up-front consideration of all these criteria. The Parameter Descriptions included as independent attachments to this document are intended to assist those who must develop the specific Data Transfer Protocols (see Section 5.0 and Section 6.0), but the protocols can be developed without a corresponding Parameter Description. The following discussion addresses issues related to specific parameters. 


\subsection{Hydraulic Head}

Hydraulic head is commonly used as a calibration target during model

development. The hydraulic head at a location is a function of many factors, such as recharge rates, hydraulic conductivity, and geology. The most important factor is that it is not solely a function of the rock at a particular location, but is a function of location. To transfer a hydraulic-head measurement from one location to another for calibration purposes, would incorrectly constrain the model, and offers no advantages in the calibration process. Therefore, the disqualification criterion, for use as a calibration target, is that regardless of other factors, hydraulic head should not be transferred from one location to another.

This is not to say that nearby hydraulic-head measurements could not be used to estimate hydraulic head at a particular location. For example, it may be necessary to estimate hydraulic head for use in a boundary-condition specification. In such an instance, it might be appropriate to estimate the hydraulic head at one location using information from other locations. However, there are factors which should be considered in doing so. Other data may indicate the hydraulic gradient and be used to estimate a value by interpolation or extrapolation. If the hydraulic gradients in the area are low, there may be little error in the estimate. However, if hydraulic gradients are relatively high, which can occur if rocks with low hydraulic conductivity are present, the estimate should reflect what is known of the hydrogeology, and the uncertainty in the estimate should be assigned accordingly. Thus, factors that affect the hydraulic conductivity of the rocks should be considered. These include some components of lithology (such as degree of welding and alteration for volcanic rocks, and propensity for dissolution or fracturing), and structural setting (such as proximity to faulting and whether in an upper or lower thrust plate). Other components of lithology, such as feldspar composition, would be unimportant. These factors need to be evaluated based on the hydrogeology in the area. There may be no disqualifying criteria, but the uncertainty assigned to the estimate should reflect the measurement technique (including accuracy of the land-surface elevation measurement/estimate), the observed variability (either random or systematic) in the measurements, changes in fluid density from one location to another, and the distance (laterally and vertically) from the measurements.

\subsection{Melt Glass Dissolution Rate}

The rate at which the melt glass dissolves (surface-area normalized glass dissolution rate) has a direct effect on the rate of release of radionuclides from the glass. This parameter is measured in the laboratory under experimental conditions (such as water chemistry, specific surface area, and temperature) that will differ from field conditions. Equations can be used to adjust the net dissolution rate for the effects of specific surface area and temperature, but it may be more difficult to account for changes in the ambient water chemistry. For example, the dissolved silica content and $\mathrm{pH}$ of water that has been in contact with volcanic rocks will be different than water in contact with carbonate rocks. In addition, the melt glass formed from, or with a large component of, carbonate rocks will have different characteristics from melt glass formed from volcanic rocks. The melt glass 
formed from basin fill materials may also have different characteristics than that from volcanic rocks, depending on the composition of the basin fill. Thus, the disqualification criterion may address the composition of the host material and the ambient groundwater chemistry. Dissolution rates can be time dependent processes with higher rates initially and slower rates following the development of weathering rinds that partially protect the remaining glass. Disqualification criteria need to be consistent with these temporal trends. However, the nature of the nuclear device may have little impact on the dissolution rate, so that no exclusion criterion based on the device may be needed.

\subsection{Bulk Hydraulic Conductivity}

The hydraulic conductivity of the rock is one of the primary parameters that determines the spatial distribution of groundwater movement; therefore, it is likely to be one of the more important parameters in CAU-scale flow models. However, hydraulic conductivity is highly variable in fractured rocks such as the volcanic and carbonate rock aquifers. Therefore, the results of tests that measure hydraulic conductivity are determined in part by the type of test, the size of the tested interval, and whether observations are available from monitoring wells located near the well that is pumped. For example, the results of single well tests often are biased by the properties of the rocks immediately adjacent to the well bore, rather than a more representative, average value. Thus, characterizing a single HSU requires numerous tests to determine the average value. This creates a different type of problem than if each measurement of a property (such as the hydraulic conductivity of the matrix) could be considered as reasonable representation of the rock. Further, 5 to 15 measurements in a single HSU may still not produce a reliable estimate, and combining of measurements from several HSUs of similar lithology may be necessary. The exclusion criteria may include restrictions that only appropriate analysis approaches be used, and that results from atypical locations and laboratory-scale measurements not be used. Although multiple well tests are believed to provide the most representative results, it would not be appropriate to exclude single-well pumping tests or packer injection tests because of the very limited number of multiple-well tests that have been performed. The evaluation of the data should consider the type and scale of the tests, as well as factors such as depth of the tested interval.

\subsection{Effective Porosity}

The effective porosity of a rock is a measure of the proportion of the volume of rock through which water most actively flows. Measurements by laboratory tracer tests performed on core of granular materials have commonly shown that the effective porosity is less than the total porosity, presumably because of preferential pathways. In homogeneous rock, the effective porosity is conceptually easy to determine. However, in very heterogeneous rock, its measurement may be difficult. For example, the BULLION Forced Gradient Experiment (FGE) can be interpreted in different ways. Interpretation using a porous media equivalent model with little heterogeneity yielded effective porosity 
values of approximately 3 to 10 percent. Some scientists have suggested that this high value for effective porosity resulted from heterogeneity, with the pumping well causing water to flow faster along pathlines other than those along which tracers moved; if this conceptual model were to be simulated, this model would yield lower values of effective porosity than previously interpreted. These scientists also suggested that if the test had been run in a partial recirculation mode, the test would have had a higher probability of yielding more representative values. A fracture-network model with variable property fractures was able to approximate the breakthrough behavior using effective porosity for the fractures of approximately $2.4 \times 10^{-5}$. Thus, the test-determined value for effective porosity is a function not only of the test location, but also the testing method and the method used to interpret it.

Data transfer of effective-porosity values is not straight-forward. In addition, there are few tests that have been performed at the NTS. Perhaps no exclusion criteria should be established for effective porosity. On the other hand, the values to be used for the CAU and other models should reflect the modeling scale and approach, including how heterogeneity is handled. In the above BULLION FGE example, the high values obtained from the approximately homogeneous model are probably too high for use in a porous media equivalent model, while those from the fracture network model are probably too low to be used in a CAU-scale, porous-media-equivalent model.

\subsection{Sorptive Behavior of Radionuclides}

Interactions between radionuclides and the rock through which they are moving tend to reduce the rate at which the radionuclides move. These interactions have typically been characterized through either batch or column sorption experiments, commonly using rock that has been crushed, increasing the surface area for sorption and diffusion into the particle interiors, and exposing fresh surfaces to the water. Thus, the laboratory-determined sorption parameters may not be representative of those that would be present in the rock. If water flows primarily through fractures, the mineralogy of the fracture face may have a dominant effect on the sorptive behavior, and not be appropriately represented in the crushed sample. Thus, there is a question about the use of laboratory data to represent field conditions.

A second issue is more directly related to the "transfer" of sorption data from outside a CAU. Sorption is affected by the mineralogy of the samples and the chemistry of the water. The YMP has performed many sorption measurements, using rock from the Yucca Mountain site and water created to mimic water from $\mathrm{J}-13$ in Jackass Flats. The mineralogy of the volcanic rocks within the CAUs is similar to, but different from, the rocks at Yucca Mountain, and the water chemistry is also different. In addition, many of the nuclear tests were conducted in basin fill materials, which are more weathered than the volcanic rocks at Yucca Mountain.

The range in distribution coefficient $\left(\mathrm{K}_{\mathrm{d}}\right)$ for a particular radionuclide, as determined by the YMP tests, is large (typically 2 or more orders of magnitude). 
Given this large range, a reasonable question is "Does it make sense to be concerned with relatively minor changes in mineralogy or water chemistry if the variance of the results is so large and the repeatability of the measurements is so poor?" Another pertinent question is "Are there situations where Yucca Mountain sorption data should not be used because they might not be conservative?" One likely environment is in carbonate rock. 


\subsection{Recommended Steps for Establishing the Data Transfer Protocol for a Parameter}

As the previous examples illustrate, the approach for using data from other locations will need to be tailored for each parameter, and perhaps for each CAU. The specific use of the data and the scale of the model may also affect the transfer process. The following sequence of steps is intended to assist in developing criteria for the data transfer process, and to provide a record of the decision-making process.

1. Describe the parameter as it pertains to its intended use. For example, the value of the modeling parameter hydraulic conductivity may change depending on the scale of modeling to be performed and whether heterogeneity is to be explicitly incorporated. Hydraulic conductivity measurements for the rock matrix may be important for simulating moisture movement in the vadose zone, but are probably not useful for evaluating saturated zone transport at the CAU scale.

2. Consider the sensitivity of predictions of the contaminant boundary to variation in the parameter value. Although the use of modeling simulations would be ideal for determining the parameter sensitivity, informed professional judgment is appropriate, especially during the early phases. If the modeling results are not likely to be very sensitive to the parameter value over reasonable ranges, there should be only minor effort in developing and implementing the Data Transfer Protocol.

3. Consider how the data are collected. The same parameter can be measured in different ways and at different scales. Evaluate the averaging volume for the measurement. The measurement technique can determine whether the measurement can be used for the intended use.

4. Identify the factors that determine or affect the parameter value. These factors will need to be compared between the measurement or sampling location, and the location of intended use. For example, the presence of zeolites in volcanic rocks has been determined to be associated with greater sorption of some radionuclides. Therefore, it would be inappropriate to use $\mathrm{K}_{\mathrm{d}}$ values determined for zeolitic rocks for non-zeolitic rocks.

5. Discuss the important considerations for using data from another source, and develop disqualification criteria. The criteria should reflect the importance of the parameter to the determination of the Contaminant Boundary and the reduction of uncertainty that might result from more 
stringent criteria. A general guideline is that the criteria should be established to eliminate data of poor quality and to eliminate inappropriate use of data.

The Data Transfer Protocol, incorporated in the data reports, analysis reports, and/or model reports, should be written with sufficient detail to allow technically qualified, but perhaps non-expert, personnel to understand the reasoning behind the criteria, and to apply them with little outside guidance.

Depending on the importance of a parameter, the Data Transfer Protocol may be short and simple, or lengthy. It is important that the above steps be used and documented, but the thoroughness of the evaluation should reflect the results of step "2." For important parameters, Parameter Description may be prepared if they would assist in preparation of the Data Documentation Packages or other documents. 


\subsection{Suggested Outline for Data Transfer Protocol}

The Data Transfer Protocols incorporated into UGTA project documents should be written according to the following outline. Although data transfer protocols may not necessarily be CAU dependent, this outline allows for the possibility that some protocols could have CAU-specific elements.

A. Title and Signature page, with the parameter name, intended use, and CAU identified, and with a revision number.

B. Body of the Data Transfer Protocol

1. Introduction - Provide additional information on the parameter, the intended use, and the CAU.

2. Parameter Description - Provide detail on the definition of the parameter, its intended use, and how it will be used in the CAU model(s).

3. Estimated sensitivity of the CAU model - Estimate the importance of the parameter, and how this was determined.

4. Measurement Techniques - Describe how the measurements were made and any impacts the measurement technique may have on the interpretation and use of the data in the context of the CAU model(s).

5. Important Factors - Identify any information that should be considered in the preparation of disqualification criteria and the use of the transferred data.

6. Criteria - Define the disqualification criteria and the reasons why they are established. 


\subsection{Conclusions}

The models that will be used to evaluate radionuclide transport are data dependent. However, because of the size of the underground testing areas and the complexity of the problem, the data set is not and will not be "complete" enough to produce a fully constrained, or unique, model. It is likely to be necessary to use information developed in similar settings to allow transport to be predicted, and to help quantify resulting uncertainty in the predictions.

This methodology is based on the UGTA strategy for addressing uncertainty. Transfer is assumed to be appropriate unless there are characteristics of the use, measurement technique, scale of measurement, or other characteristics that would make use questionable or would produce non-conservative results. Rather than defining "acceptance criteria," "disqualification criteria" are to be established in the Data Transfer Protocol. The Data Transfer Protocols must provide sufficient information so that authors of the Data Documentation Packages can successfully interpret and apply the criteria.

Because the transfer of data depends on the intended use, the source of the information, geohydrological and chemical settings, and controls on the parameter value, a simple recipe for transferal cannot be developed. The methodology presented is flexible but requires consideration and documentation of these factors. 


\subsection{References}

FFACO, see Federal Facility Agreement and Consent Order.

Federal Facility Agreement and Consent Order. 1996 (as amended). Agreed to by the State of Nevada, the U.S. Department of Energy, and the U.S. Department of Defense. 


\section{Appendix A}

Establishing the Similarity of Geologic Settings: A Formal Statistical Approach 


\section{A.1.0 Introduction}

The second factor listed at the beginning Section 4.0 of the main document that affects the transferability of data is the "similarity of donor and receptor HSUs, with respect to relevant characteristics for the variable of concern." Similarity is a rather subjective concept, so this section provides some guidance for formally establishing similarity between geologic settings that can be used in the initial screening of whether data from another location should be considered for transfer. This screening process is applicable to both the reject-unless-accepted and the accept-unless-rejected approaches described in Section 3.0 of the main document.

It should be pointed out that formally establishing similarity between two locations should generally not be applied directly to the parameter of interest. If sufficient data exist to formally test the similarity of a parameter of interest in two different locations, then it is quite likely that enough data exist from either location that the data from the second location will add little to the understanding of parameter values at the first location. Rather, the formal testing of similarity should be conducted when data are scarce for the parameter of interest, but a significant amount of data exist from both locations on a parameter or material property that the parameter of interest is expected to be dependent on or correlated with. 


\section{A.2.0 Similarity}

Transferability based on similarity is to some extent always a leap of faith. Furthermore, similarity is a continuum measure rather than a discrete measure. The same quantity of data necessary to rigorously establish the similarity of a given attribute at two locations would generally serve to characterize each location with respect to that attribute. For this reason, it is implicitly assumed in the following discussion that the data being used to evaluate similarity are not for the parameter to be transferred. The following discussion uses porosity as an example. Therefore porosity data would not be used in the similarity evaluation. Transferring data is almost always an implicit decision to accept the creation of some unquantified degree of uncertainty.

Transferability rests upon the truth or falsity of the apparent tautology that samples from similar units will be more alike than samples from different units. When the attribute being considered for transfer is a primary component of the classification scheme, then the tautology is likely to hold. However, if the attribute is a minor component of the classification scheme, or in the worst case, not a component at all, then transferability must be considered speculative. When considering geologic units, such as lithostratigraphic units, porosity is not a defining attribute. Therefore the degree to which similar lithostratigraphic units have similar porosities is unknown and must be established by testing or explicitly embracing unsupported assumptions. Conversely, hydrostratigraphic units, as defined by Seaber (1988 and amended in 1992), are clearly defined by the similarity of porosity (and other aspects of the void space).

A hydrostratigraphic unit is a body of rock distinguished and characterized by its porosity and permeability. A hydrostratigraphic unit may occur in one or more lithostratigraphic, allostratigraphic, or lithodemic units and is unified and delimited on the basis of its hydrologic characteristics and interstices (Seaber, 1992).

Similarity is a common basis for many classification schemes, ranging from the largely qualitative North American Stratigraphic Code, to primarily quantitative approaches such as Lance and Williams (1967), Oliver and Webster (1989), Bourgault et al. (1992), Grunsky and Agterberg (1992), and Gill (1993).

A generalized coefficient of similarity was developed by Gower (1971) 


$$
S_{i j}=\frac{\sum_{k-1}^{r}\left(1-\left|z_{i k}-z_{j k}\right| / r_{k}\right) w_{i j k}}{\sum_{i=1}^{p} w_{i j k}}
$$

where $z_{\mathrm{ik}}$ and $z_{\mathrm{jk}}$ are observed values of the $k$ th property of individuals $i$ and $j$, and $r_{\mathrm{k}}$ is the range of the $k$ th property, and $w_{\mathrm{ijk}}$ is a weight assigned to comparison on the $k$ th property.

When a property is qualitative, the expression $\left(1-\left|z_{i k}-z_{j k}\right| / r_{k}\right)$ reduces to either a binary zero or one in the case of mutually exclusive categorical properties or to a value ranging from zero to one in the case of ranked or ordered multistate properties. A geologic example of mutually exclusive, categorical properties is the time honored groupings of igneous, metamorphic, and sedimentary. An example of ranked multistate properties might be degrees of welding in volcanic rocks (i.e., non-welded, partially welded, moderately welded, welded and vitrophyres). Thus a partially welded rock would be more similar to a moderately welded rock than it would be to a welded rock. The numeric values based on order or ranking need not be equidistant so the dissimilarity between a non-welded and partially welded rock may be greater than the dissimilarity between a partially welded and a moderately welded rock.

Similarity can be most easily thought of as proximity in multidimensional space. The similarity matrix $s_{i j}$ is converted to dissimilarity $\left(d_{\mathrm{ij}}\right)$ by

$$
d_{i j}=\left[2\left(1-s_{i j}\right)\right]^{1 / 2}
$$

The relevance of similarity and dissimilarity to data transference arises from the reasonable and intuitively appealing assertion that similarity based on a large number of dimensions (e.g., lithology, alteration history, tectonic history) believed to be related to the attribute of interest (e.g., porosity) support an assertion of similarity in the attribute of interest. The difficulty of directly measuring many of the important parameters in hydrogeology has been a driving force in the widespread application of this reasoning (Tidwell and Wilson, 2002). The weight and multiplier system proposed in Appendix B is a pragmatic method of applying similarity to constrain estimates of porosity when the amount and quality of directly applicable data is inadequate to support more rigorous approaches. 


\section{A.3.0 Statistical Tests}

Quantitative evaluation of the similarity of two datasets drawn from geographically separate units can be facilitated by population and spatial statistical tests. Similarity in population alone may be adequate for some purposes.

However, for many modeling applications similarity in spatial structure may be necessary. Similarity in terms of population statistics needs to be evaluated prior to considering spatial statistics for the simple reason that spatial similarity is meaningless unless population similarity exists.

In Numerical Recipes, Press et al. (1990) address one of the fundamental problems which must be considered when contemplating data transfer

... Are the two sets drawn from the same distribution function, or from different distribution functions? Equivalently, in proper statistical language, Can we disprove, to a certain required level of significance, the null hypothesis that two dataset are drawn from the same population distribution function?" Disproving the null hypothesis in effect proves that the datasets are from different distributions. Failing to disprove the null hypothesis, on the other hand, only shows that the datasets can be consistent with a single distribution function. One can never prove that two datasets come from a single distribution, since (e.g.) no practical amount of data can distinguish between two distributions which differ only by one part in $10^{10}$.

The following summary is largely drawn from Numerical Recipes (Press et al., 1997) and additional details can be obtained from that source. The most common statistical tests used to evaluate the "sameness" of two populations (e.g., the property values taken from two discrete locations) are the $t$-test and the $F$-test. Evaluation of the comparability of the means can be done using the $t$-test

$$
\begin{gathered}
t=\frac{\bar{x}_{a}-\bar{x}_{b}}{s_{D}} \\
\text { where } s_{D}=\sqrt{\frac{\sum_{a}\left(x_{i}-\bar{x}_{a}\right)^{2}+\sum_{b}\left(x_{i}-\bar{x}_{b}\right)}{\mathrm{N}_{a}+\mathrm{N}_{b}-2}\left(\frac{1}{\mathrm{~N}_{a}}+\frac{1}{\mathrm{~N}_{b}}\right)}
\end{gathered}
$$

is the standard error of the difference of the means, the subscripts $a$ and $b$ designate separate datasets, $x$ designates an individual sample, and $N$ is the number of samples in a dataset. The significance of the value of $t$ is evaluated for Student's distribution with $\mathrm{N}_{a}+\mathrm{N}_{b}-2$ degrees of freedom. The significance 
ranges from zero to one, and is the probability that solely by chance, $|t|$ could equal or exceed the calculated value assuming that distributions $a$ and $b$ have the same mean.

While the $t$-test can be used to compare the variance of two populations, the two tailed $F$-test is preferred. The $F$-test is used to either accept or reject a hypothesis on the comparability of the variance of two samples.

$$
\begin{array}{r}
H_{\mathrm{o}}: \sigma_{a}^{2}=\sigma_{b}^{2} \\
H_{a}: \sigma_{a}^{2} \neq \sigma_{b}^{2}
\end{array}
$$
where $\sigma_{a}^{2}$ is the population variance for dataset $a$ and $\sigma_{b}^{2}$ is the population
variance for dataset $b$. The two tailed $F$-test statistic is

$$
F=\frac{s_{a}^{2}}{s_{b}^{2}}
$$

where $s_{a}^{2}$ is the larger sample variance and $s_{b}^{2}$ is the smaller sample variance. The rejection region (that is, when the hypothesis of comparable variance must be rejected) is when the calculated statistic exceeds the tabulated value $F>F_{a / 2}$ given the relevant degrees of freedom for $v_{\mathrm{a}}$ (the degrees of freedom for the numerator sample variance) and $v_{\mathrm{v}}$ (the degrees of freedom for the denominator sample variance). $\alpha / 2$ is the probability of erroneously rejecting $H_{0}$ when $H_{0}$ is true.

Both the t-test and the F-test have the following assumptions:

1. Both sampled populations have normal distributions

2. The samples are random and independent.

An alternative method of estimating equivalence for two datasets is the Kolmogorov-Smironov (K-S) test. The K-S test is

$$
D=\max _{-\infty<x<\infty} s_{a}(\mathrm{x})-s_{b}(\mathrm{x})
$$

where $S_{a}(x)$ and $S_{b}(x)$ are unbiased estimators of the cumulative distribution functions of the probability distributions $a$ and $b$. The significance of nonzero values of $D$ is approximated by

$$
\text { Probability }(\mathrm{D}>\text { observed })=Q_{K S}\left(\sqrt{\frac{N_{a} N_{b}}{N_{a}+N_{b}} D}\right)
$$


where $Q_{K S}(\lambda)=2 \sum^{\infty}(-1)^{j-1} \mathrm{e}^{-2 j^{2 \lambda 2}}$ and $N_{a}$ and $N_{b}$ are number of data points in the datasets being compared.

A particularly attractive feature of the K-S test is that it makes no assumptions about the type of probability distributions $a$ and $b$. However two cautions need to be observed: (1) the K-S test is least sensitive in the tails of the distributions and (2) the minimum number of samples needed can be estimated from $N_{e}=\frac{N_{a} N_{b}}{N_{a}+N_{b}}$ where $\mathrm{N}_{\mathrm{e}} \geq 4$.

Some other statistical tests useful for comparing two datasets include the Wald-Wolfowitz runs test, the Mann-Whitney $U$ test, the Kruskal-Wallis ANOVA and Median test, the Cochran $Q$ test, the Friedman ANOVA test, and the Kendall Concordance test. Discussion of these methods is beyond the scope of this appendix and the data requirements for these tests may make application difficult. 


\section{A.4.0 Spatial Statistics}

If the datasets can meet the basic tests of population similarity, then evaluation of similarity of spatial structure may be warranted. The general spatial model for random variables as defined by Cressie (1993) is $s \varepsilon \mathrm{R}^{d}$ and is a generic data location in $d$-dimensional Euclidean space, $Z(s)$ is a potential datum at location s. $\mathrm{s}$ is varied over index set $D \subset R^{d}$ to generate the random field or process $\{Z(s): s \subset D\}$; a realization of which is denoted $\{z(s): s \subset D\}$.

For instance, porosity can be considered a random variable and treated accordingly. Random variables such as porosity are commonly correlated in space, and the nature of the correlation has significant implications for flow and transport. The spatial correlation of a random variable can be described in a number of ways with current practice focusing on the variogram.

The classical estimator of the variogram, from Matheron (1962), cited by Cressie, 1993 is

$$
\hat{2 \gamma}(h) \equiv \frac{1}{|N(\mathrm{~h})|} \sum_{N(\mathrm{~h})}\left(Z\left(s_{i}\right)-Z\left(s_{j}\right)\right)^{2}
$$

where $\mathrm{s}_{i}$ is a spatial location separated from location $\mathrm{s}_{j}$ by distance $\mathrm{h}, Z(\mathrm{~s})$ is a datum at location s, and $N$ is the number of data separated by distance $h$.

The data requirements for rigorous variogram estimation include

1. Multiple samples

2. Correct spacing between samples

3. Support (sample scale) the same as intended use

4. Samples are specific to a unit (population), not mixed

5. Sampling method is uniform and unbiased

It cannot be overstated that an adequate number of properly spaced, correctly scaled, unbiased samples are a requisite if these methods are to deliver the promised statistical rigor. When used with lesser quality data and more assumptions, the methods can still provide useful models, but rigor will be lost. In particular, the problem of support or measurement scale has been recognized important: 
There are a variety of mathematical methods for adjusting a distribution so that its variance will be reduced while its mean remains unchanged. Unfortunately, all of these depend on unverifiable assumptions about how the distribution changes as the support increases; they also require knowledge of certain parameters that are very difficult to estimate precisely.

This problem of the discrepancy between the support of our samples and the intended support of our estimates is one of the most difficult we face in estimation. (Isaaks and Srivastava, 1989)

A relatively parsimonious description of a random field consists of a mean, variance, correlation lengths and orientation of the correlation anisotropy ellipsoid. The correlation lengths $\left(\lambda_{x}, \lambda_{y}, \lambda_{z}\right)$, anisotropy of correlation $\left(\lambda_{x}: \lambda_{y}: \lambda_{z}\right)$ and one of the three angles of rotation $\left(\alpha_{1}, \alpha_{2}, \alpha_{3}\right)$ controlling the orientation of the correlation ellipsoid are illustrated in Figure A.4-1.

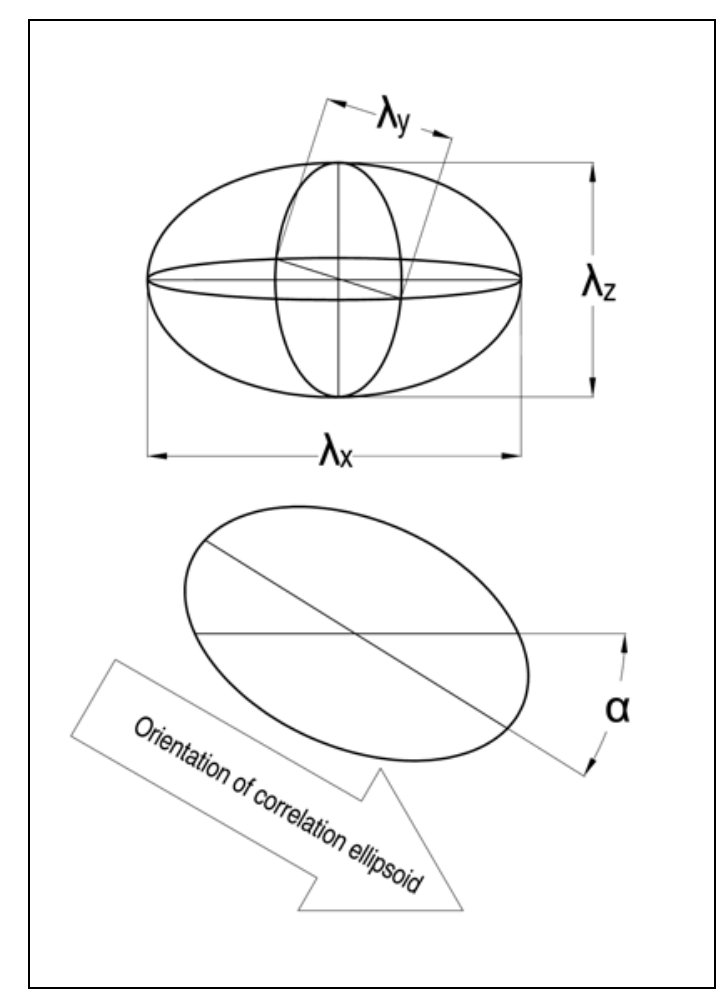

\section{Figure A.4-1 \\ Ellipsoid of Spatial Correlation}

Comparison of the correlation lengths and angles of rotation will demonstrate the degree of similarity. However, if enough data exist to estimate these parameters, then the data are likely to be sufficient to directly support the modeling. For this reason, formal testing of similarity is probably best applied to a parameter or material property that the parameter of interest is expected to be dependent on or correlated with rather than directly on the parameter of interest. 


\section{A.5.0 References}

Bourgault, G., D. Marcotte, and P. Legendre P. 1992. "The Multivariate (Co)Variogram as a Spatial Weighting Function in Classification Methods." In Mathematical Geology, Vol. 24, No. 5.

Cressie, N.A.C. 1993. Statistics for Spatial Data, Revised Edition. New York, NY: John Wiley and Sons, Inc.

Gill, D. 1993. "Discrimination of Sedimentary Facies by Association Analysis." In Mathematical Geology, Vol. 25, No. 4.

Gower, J.C. 1971. "A General Coefficient of Similarity and Some of Its Properties.” In Biometrics, Vol. 27, pp. 857-871.

Grunsky, E.C., and F.P. Agterberg. 1992. "Spatial Relationships of Multivariate Data." In Mathematical Geology, Vol. 24, No. 6.

Isaaks, E.H. and R.M. Srivastava. 1989. Applied Geostatistics. New York, NY: Oxford University Press.

Lance, G.N., and W.T. Williams. 1967. "Mixed-Data Classificatory Programs, I. Agglomerative Systems." In The Australian Computer Journal, November.

Matheron, G. 1962. "Traite de Geostatistique Appliquee, Tome I. In Memoires du Bureau de Recherches Geologiques et Mineres, No 14. In Editions Bureau de Recherche Geologiques et Mineres. Paris, France.

Oliver, M.A., and R. Webster. 1989. "A Geostatistical Basis for Spatial Weighting in Multivariate Classification." In Mathematical Geology, Vol. 21, No. 1.

Press, W.H., B.P. Flannery, S.A. Teukolsky and W.T. Vetterling. 1990. Numerical Recipes in Pascal, The Art of Scientific Computing. New York, NY: Cambridge University Press.

Press, W.H., S.A. Teukolsky, W.T. Vetterling, and B.P. Flannery. 1997. Numerical Recipes in C, The Art of Scientific Computing Second Edition. New York, NY: Cambridge University Press.

Seaber, P.R. 1988. "Hydrostratigraphic Units," In Hydrogeology. W. Back, J.S. Rosenshein, and P.R. Seaber, eds. Boulder, CO: Geological Society of America. 
Seaber, P.R. 1992. Proposed Addition to the North American Code of Stratigraphic Nomenclature (Draft).

Tidwell, V.C., and J.L. Wilson. 2002. "Visual Attributes of a Rock and Their Relationship to Permeability: A Comparison of Digital Image and Minipermeameter Data.” In Water Res. 38(11) 1261. 
Appendix B

Recommended Approach for Determining Emphasis Given to Transferred Data 


\section{B.1.0 Overview}

The recommended approach for determining the relative emphasis that should be assigned to the data is to use a weight and multiplier system. This approach is also recommended for datasets obtained within a CAU of interest because, even though all such data will come from a relevant geologic setting, some CAU-specific datasets should rightfully be assigned greater emphasis than others due to differences in measurement methods, data quality, and documentation quality. The weight and multiplier system is quite subjective, which is not particularly appealing from a scientific perspective, but it is consistent with the subjective nature of the data transferability process. The system and process ultimately rely heavily on the documentation of the rationale for weights and multipliers, which essentially provides the rationale for the transfer of data.

In the weight and multiplier system, weights are determined for datasets based on the relevance of the geologic setting to the CAU, and multipliers (factors that the total weight score is multiplied by to obtain an overall score) are assigned based on the measurement method, the quality of data reduction and analyses, and documentation quality. The overall score for a given dataset is the sum of the weights multiplied by the product of the multipliers. Datasets with poor geologic setting relevance, while they may still be transferred, are assigned lower weights so they ultimately "count less" in determining the final parameter distribution used in CAU-scale modeling. Likewise, datasets associated with measurement methods having large uncertainties or inappropriate scales, or datasets of low-quality or having poor documentation are assigned lower multipliers so that they count less in the final CAU-scale application.

The rationale for using both weights and multipliers is that the geologic setting relevance and the data quality are two separate criteria that are largely independent. Weights are used for geologic setting relevance because this is an overriding consideration in most cases. Datasets obtained from the best possible measurement method and having high-quality analyses and documentation should nevertheless be rejected if the geologic setting is inappropriate. In a system based entirely on weights, such datasets could potentially still be assigned a relatively high weight score even though they are not appropriate for the CAU of interest. Multipliers are assigned after weights are determined so that appropriate credit can be taken for the measurement method and data/documentation quality. Although datasets will seldom be rejected outright at this stage, very low multipliers can be assigned because of a highly uncertain measurement method or very poor documentation. The weights and multipliers have relative scales rather than absolute scales. For instance, if there are only two datasets being considered, then multipliers of 1.0 and 0.5 for these sets will have the same effect as multipliers of 0.2 and 0.1 , respectively (i.e., the first dataset has a multiplier twice that of the 
second set in both cases). These relative scales make it imperative that the rationale for weights and multipliers be clearly documented so that if additional data are later considered for transfer, they can be scored in a manner consistent with the previously transferred data.

This approach of "graded" transferability recognizes that most transfer decisions will not be a simple "yes or no", but rather there will be varying degrees of relevance of datasets to a given CAU, as well as varying degrees of measurement and documentation quality. It also recognizes that, while the goal is to make objective decisions regarding data transferability, there are almost always subjective elements involved in these decisions.

Section B.2.0 provides guidance for determining the relevance of geologic settings of datasets to NTS CAUs. Section B.3.0 provides guidance for determining multipliers by ranking measurement methods, and evaluating data reduction and documentation quality. Section B.4.0 addresses combining weights and multipliers to arrive at overall scores. Section B.5.0 provides guidance for documenting the implementation of this process. Section B.6.0 provides a discussion of the use of weights and multipliers in downstream analyses. Note that the guidance provided in these sections can be deviated from if there are justifiable reasons for doing so. However, such deviations should be documented in the rationale for the selection of weights and multipliers (see Section B.5.0). Attachment A (Section 2.0) to this document provides a more detailed discussion of this approach for porosity data. 


\section{B.2.0 Weights (Relevance of Geologic Setting)}

Note that the overall weight for a dataset is the sum of the weights for each of the three criteria below.

\section{Rock Type}

If the rock type is significantly different from the type in the CAU, then the data should not be considered for transferability unless there is some unusual circumstance. That is, an overall weight score of zero should be assigned so that regardless of the measurement method or data quality, the dataset is rejected. If the rock type is the same, then the analyst should proceed to step 2. However, the analyst may reject data at this point even if it is from the same rock type because of an obvious difference in geology. For instance, sandstone data should not be considered for limestone settings even though both rocks are nominally sedimentary. Also, glassy basalt data should not be considered for devitrified lava settings even though both rocks are volcanic lavas.

\section{Deposition and Alteration History}

Similarities and differences in deposition history and geochemical/hydrothermal alteration should be evaluated and weights assigned using a sliding scale of 0 to 0.7 , with 0.7 indicating a very similar deposition and alteration history. Any score near zero should prompt the analyst to question whether the data should even be considered for transferability. This step represents a second point where data could potentially be rejected, with the examples above applying here as well. However, once data makes it past this step, it should no longer be rejected outright; instead, it should be assigned a low weight if it has questionable relevance to the CAU. Further guidance on how to assign sub-weights within the 0 - to -0.7 sliding scale is not provided because of the multiplicity and complexity of considerations involved, as well as the desire to give the analyst(s) flexibility for a wide range of potential situations. However, the burden is on the analyst to document the rationale for the assigned weight.

\section{Structural Setting and Mechanical Alteration}

Similarities and differences in structural setting and mechanical alteration should be evaluated and weights assigned using a sliding scale of 0 to 0.3 , with 0.3 indicating a very similar structural setting. In this case, a score near zero should not necessarily disqualify a dataset from consideration. Again, the analyst is encouraged to use the formal statistical methods of addressing similarity discussed in Appendix A to help determine and document a score.

Note: If CAU-scale models are developed that include domains of different rock types, deposition/alteration histories, and/or structural settings, individual datasets 
can be considered for each model domain and weights can be assigned on a case-by-case basis for each domain. For instance, given the concept of multiple HSUs in flow and transport models, a particular dataset could be "transferred" to different HSUs, with a different weight assigned to each HSU. This same approach can be extended to situations were HSUs contain multiple rock types or multiple deposition/alteration histories; the data could be transferred with different weights assigned to different portions of the HSU. Also, if different structural features are distinguished in model domains (e.g., faulted and unfaulted regions), datasets could be weighted differently for these different regions. 


\section{B.3.0 Multipliers (Measurement Method and Quality of Analyses and Documentation)}

Note that the overall multiplier for a dataset is the product of the multipliers for each of the three criteria listed below.

\section{Measurement Method}

Measurement method considerations include the quality and uncertainty of the method as well as the scale of the measurement. The approach taken for relative rankings of measurement methods should be documented by the analyst. Guidance for numerical values of multipliers for porosity and matrix diffusion parameters, respectively, are provided in Attachments A and B. Larger numbers should reflect what is considered to be a "better" measurement.

\section{Data Reduction and Analysis Methods}

Data reduction and analysis methods are evaluated independently of the type of measurement being evaluated. However, the analyst must be familiar with state-of-the-art methods in order to make an evaluation. The following multipliers apply (a sliding scale can be employed to allow for a continuum of scores):

- Current and widely-accepted data reduction and analysis method with reputable and traceable calibration information -1.0

- Good method, but calibration information is suspect -0.5

- Based on older or less widely-accepted methods, but calibration information is good -0.5

- Both methods and calibration information are suspect -0.2

Note: Score for any poorly-analyzed dataset can be raised if the raw data are available and are re-analyzed using state-of-the-art methods. However, it is not possible to raise a score above 0.5 for datasets with poor calibration information (unless other information can be used to effectively "recalibrate" the measurements).

\section{Quality of Documentation}

Documentation quality is also evaluated independently of the type of measurement being evaluated. The following multipliers apply (a sliding scale can be employed to allow for a continuum of scores): 
- $\quad$ Good (thorough, easy to follow, traceable) -1.0

- Appearing in a reputable journal or peer-reviewed report, but otherwise lacking in thoroughness -0.6

- $\quad$ Poor -0.3

Note: It is often the case that poor documentation results in the inability to evaluate the data reduction and analysis method (including the calibration information) for a given dataset. Thus, poorly-documented estimates can, in effect, be doubly penalized as a result of both poor documentation scores and poor data reduction and analysis scores. 


\section{B.4.0 Overall Scores}

The overall score for a given dataset is taken to be the sum of the weights multiplied by the product of the multipliers. For example, if a dataset is assigned component weight scores of 0.5 and 0.2 (for categories 2 and 3, respectively, in Section B.2.0), the total weight score is 0.7 . If this dataset is then assigned multipliers of $0.8,1.0$, and 0.6 (for the three respective categories in Section B.3.0), the total multiplier score is $0.8 \times 1.0 \times 0.6=0.48$. The overall score is then the product of the weight and multiplier scores, or $0.7 \times 0.48=0.336$. 


\section{B.5.0 Documentation}

For each dataset that is considered for use in CAU-scale flow and transport modeling, the following information should be documented:

- For rejected data, rationale for rejection (i.e., rationale for assigning a total weight score of zero).

- For accepted data, a summary of the data.

- The scores for each weight and multiplier category listed in Sections B.2.0 and B.3.0.

- The rationale for the scores for each weight and multiplier category listed in Sections B.2.0 and B.3.0.

- The overall weight and multiplier score for the dataset.

A format for documenting this information is not specified, but each of the above elements should be addressed.

Documentation is very important because it is here that the weight and multiplier scores are justified. Assigning the weights and multipliers using the guidance and relative scales provided in Sections B.2.0 and B.3.0 forces the analyst to consider the applicability and relative merits of each dataset. It is not so important that the analyst follow this guidance to the letter, but rather it is important that the analyst documents and justifies the assigned weights and multiplier scores.

Documentation ensures that the analyst's decisions are traceable and easy to review. It also ensures that if additional data are later considered for transfer, these data are scored in a manner consistent with the scoring of previously transferred data. 


\section{B.6.0 Use of Weights and Multipliers in Downstream Analyses}

The data that are transferred to a given CAU (as well as CAU-specific data) can be used in many different ways in CAU-scale modeling. It is not the purpose of this document to recommend or exhaustively consider all the possible uses of data or the ways in which the combined weight and multiplier scores should be incorporated into these uses. Rather, a specific and relatively common usage of data is considered, and the manner in which combined weight and multiplier scores are incorporated into other uses can be inferred from this discussion. Specific examples for porosity and matrix diffusion parameters are provided in Attachments A and B, respectively.

It is a common practice to compile cumulative distribution functions (CDFs) of parameter values that are randomly sampled in Monte-Carlo fashion in large-scale models to simulate overall system performance. These CDFs are intended to capture the variability and uncertainty associated with parameter values within the model domain. The large-scale models are typically executed many times so that representative sampling of the component CDFs is accomplished. Finally, a CDF of the model output/predictions is constructed from the multiple model runs to allow an assessment of uncertainty in overall system performance.

The suggested method of incorporating the combined weight and multiplier scores into a CDF is best illustrated by example. We assume that there are 3 datasets from which a total of 10 values of a given parameter have been obtained to represent a given CAU (this may be from only a certain HSU within the CAU, rather than a bulk value representing the entire $\mathrm{CAU}$ ). The three datasets are generically described as follows (the values associated with each separate measurement are listed in parentheses):

- A laboratory dataset with four separate measurements on samples taken directly from the CAU $(2,3,7,7)$.

- A field dataset consisting of three measurements taken outside the CAU but in what is considered a similar rock type $(2,8,5)$.

- A field dataset consisting of three measurements taken from within the CAU $(2,6,6)$.

Without going into the details of how and why, the combined weight and multiplier scores in Table B.6-1 are assigned to the data. Note that the combined scores for the CAU-specific laboratory measurements are all assigned the same value because these measurements are assumed to be conducted as part of a single 
set of measurements (for this example) using the same analysis method. They are also assumed to be documented in a single report. Using the same combined score for multiple measurements generated within a single study is an acceptable practice when CAU-specific measurements are made in the study. When multiple non-CAU measurements are made within a given study, the weight values may vary for the different measurements because the geologic setting of the measurements may have different relevance to the CAU of interest, but the multipliers should be the same (because the same methods and documentation presumably apply to all measurements). Similarly, when measurements on CAU-specific materials are obtained in different investigations and at different times, the weight scores should be the same, but different multipliers can apply because of differences in analytical methods or documentation. Finally, when measurements are obtained on non-CAU materials in different studies and at different times, both the weight scores and multipliers can be different.

Table B.6-1

\section{Combined Weight and Multiplier Scores for Hypothetical Parameter Data}

\begin{tabular}{|c|c|c|}
\hline & Parameter Value & Combined Score \\
\hline \multirow{4}{*}{$\begin{array}{c}\text { CAU Lab } \\
\text { Measurements }\end{array}$} & 2 & 0.5 \\
\hline & 3 & 0.5 \\
\hline & 7 & 0.5 \\
\hline & 7 & 0.5 \\
\hline \multirow{3}{*}{ Non-CAU Field Data } & 2 & 0.3 \\
\hline & 8 & 0.9 \\
\hline & 5 & 0.6 \\
\hline \multirow{3}{*}{ CAU Field Data } & 3 & 1.0 \\
\hline & 6 & 0.7 \\
\hline & 6 & 1.2 \\
\hline
\end{tabular}

In Table B.6-1, the CAU field data were apparently obtained using different analysis methods or had different documentation quality because the scores are different. In the case of the non-CAU measurements, the scores are different either because of differences in the relevance of the geologic setting, differences in the analysis methods, differences in documentation quality, or any combination of these differences.

To construct a CDF from the data and combined scores of Table B.6-1, it is necessary to sort the parameter values in ascending order and then add the scores in cumulative fashion for each successive value. The cumulative score for each value is then divided by the sum of the scores for all values to obtain an estimate of the cumulative probability for that parameter measurement. Thus, the probability of each parameter value is proportional to its combined score. The results of manipulating the data from Table B.6-1 in the manner indicated above are shown in Table B.6-2. Figure B.6-1 shows the resulting CDF of parameter values. 
Table B.6-2

Results of Manipulation of Data in Table B.6-1 to Obtain Cumulative Distribution Function of Parameter Values

\begin{tabular}{|c|c|c|c|}
\hline $\begin{array}{c}\text { Sorted Parameter } \\
\text { Value }\end{array}$ & Combined Score & Cumulative Score & Cumulative Probability \\
\hline \hline 2 & 0.3 & 0.3 & 0.045 \\
\hline 2 & 0.5 & 0.8 & 0.119 \\
\hline 3 & 0.5 & 1.3 & 0.194 \\
\hline 3 & 1.0 & 2.3 & 0.343 \\
\hline 5 & 0.6 & 2.9 & 0.433 \\
\hline 6 & 0.7 & 3.6 & 0.537 \\
\hline 6 & 1.2 & 4.8 & 0.716 \\
\hline 7 & 0.5 & 5.3 & 0.791 \\
\hline 7 & 0.5 & 5.8 & 0.865 \\
\hline 8 & 0.9 & 6.7 & 1 \\
\hline
\end{tabular}

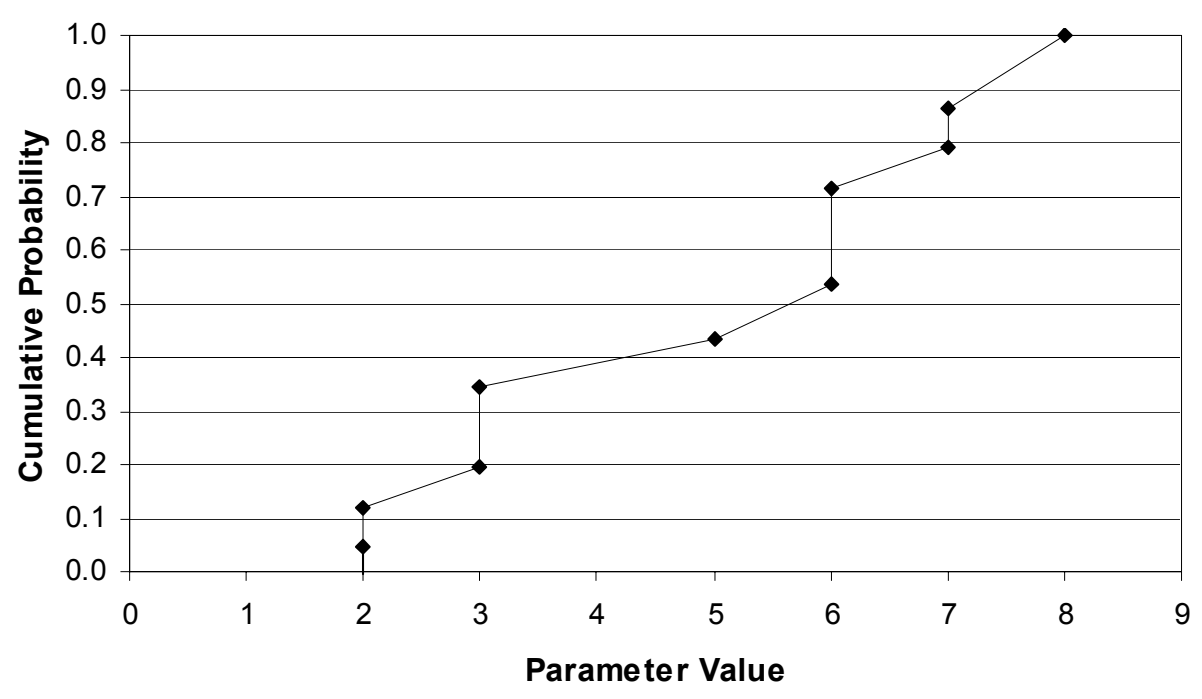

Figure B.6-1

Cumulative Distribution Function Associated With the Data of Table B.6-1

For this example, the CDF does not appear to be heavily influenced by any single measurement or set of measurements. However, in some cases, a single "very good" measurement may have a combined score equal to or greater than the sum of scores of all the other available data. In such cases, there will be a large jump in the CDF at the value corresponding to the "good" measurement.

This example also serves to illustrate the value of determining combined weight and multiplier scores for CAU-specific data in addition to non-CAU data. Even 
though the scores for CAU-specific data are not required to determine data transferability, they are essential for establishing a representative CDF.

If another method of data usage is employed in CAU-scale modeling, then the combined weight and multiplier scores should be used in some other manner to "weigh" the available datasets appropriately in the final analysis. The method(s) of translating combined scores into some measure of variability and/or uncertainty should be documented appropriately.

Finally, there are many considerations of variability and uncertainty in parameter values that are not effectively captured by the weight and multiplier system and that go beyond the scope of this document but should be addressed and documented by the analyst. For instance, because weights and multipliers are assigned using relative scales, a CDF generated with only a few data points, data of questionable relevance, and poor-quality measurement methods might look exactly the same as one with many data points, CAU-specific data, and high-quality measurements. However, even though these CDFs may look essentially the same, the uncertainty should be far greater and the confidence far less in the former distribution than the latter one. In general, uncertainty in distributions of parameter values should be considered greater for (1) less total data points, (2) less CAU-specific data, and (3) smaller values of the sum of combined scores divided by the total number of data points. 
Attachments

Parameter Descriptions 
Attachment A

Property Description - Porosity 


\section{Table of Contents}

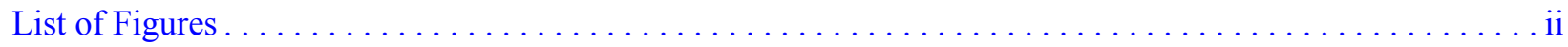

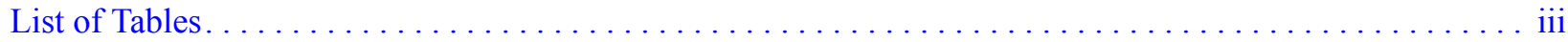

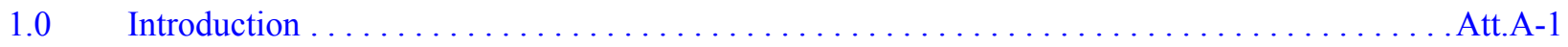

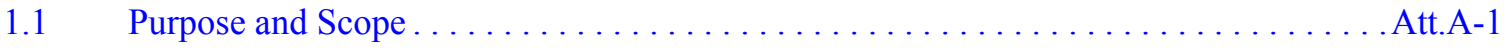

1.2 Definitions . . . . . . . . . . . . . . . . . . . . . . . . . . . Att. 1

1.3 Relationships Between the Different Types of Porosity . . . . . . . . . . . . Att.A-4

$1.4 \quad$ How Each Type of Porosity Affects Contaminant Transport . . . . . . . . . . . . . Att.A-4

$2.0 \quad$ Porosity Measurements . . . . . . . . . . . . . . . . . . . . . . . . . . . . . . .

$2.1 \quad$ Laboratory Porosity Measurements . . . . . . . . . . . . .

2.1.1 Matrix Porosity in Consolidated Media ........................

2.1.2 Matrix Porosity in Unconsolidated Media .......................Att.A-9

2.1.3 Laboratory Measurements of Other Types of Porosity . . . . . . . . . . Att.A-10

$2.2 \quad$ Field Porosity Measurements. . . . . . . . . . . . $\ldots \ldots \ldots \ldots \ldots \ldots \ldots$ Att.A

2.2.1 Geophysical Logs. .............................. Att.11

2.2.2 Hydraulic and Tracer Testing Methods . . . . . . . . . . . . . . . . Att.A-14

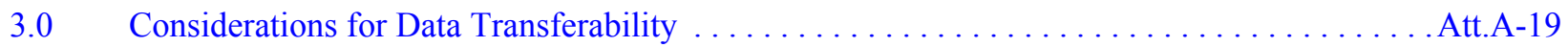

$3.1 \quad$ Geologic Setting. . . . . . . . . . . . . . . . . . . . . . . . . . . . . . . Att.A-19

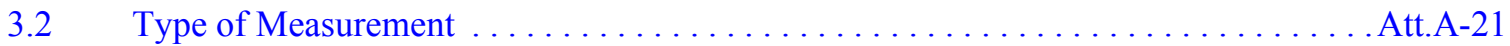

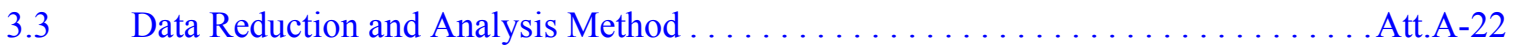

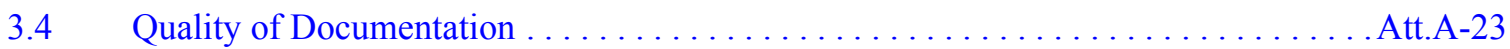

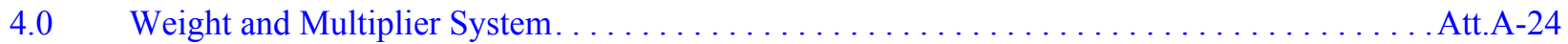

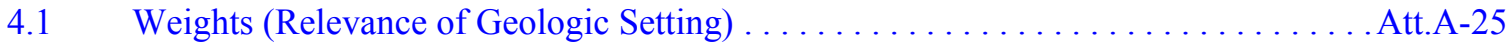

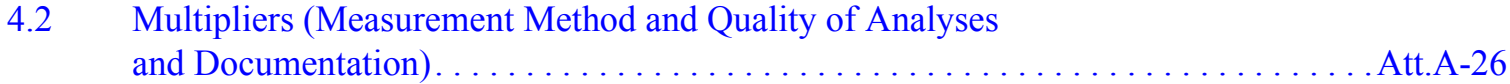

4.3 Overall Scores . . . . . . . . . . . . . . . . . . . . . . . . . . . . . . . . Att.A-29

$5.0 \quad$ Documentation Requirements . . . . . . . . . . . . . . . . . . . . . . . . . Att.A-30

6.0 Use of Weights and Multipliers in Downstream Analyses . . . . . . . . . . . . . . . . Att.A-31

$7.0 \quad$ References . . . . . . . . . . . . . . . . . . . . . . . . . . . . . . . . . . . Att.A-35 


\section{List of Figures}

Number

1-1 Depiction of the different types of porosity in a fractured rock. Blue is effective flow porosity, red is transport storage porosity, and green is disconnected storage porosity. Although it is shown to be the case here, effective flow porosity in fractured rock is not necessarily confined to fractures. . . . . . . . . . . . .

1-2 Depiction of the different types of porosity in unconsolidated porous media. Blue is effective flow porosity, red is transport storage porosity, and green is disconnected storage porosity. Note that this depiction is not intended to preclude any spatial distribution of transport storage porosity and disconnected storage porosity in unconsolidated media. . . . . . . . . . . .

1-3 Relationships between the different types of porosities. The gray area is matrix porosity, and the white area is porosity that is outside the matrix. Note that the relative volumes of regions are not intended to represent any particular type of flow system or geologic media. . . . . . . . . . . . . . . . $6 \ldots \ldots \ldots \ldots$ Att.A

6-1 Cumulative Distribution Function Associated With the Porosity Data of Table6-1 


\section{List of Tables}

Number

Title

Page

6-1 Combined Weight and Multiplier Scores

for Hypothetical Matrix Porosity Data . . . . . . . . . . . . . . . . . . . . . Att.A-32

6-2 Results of Manipulation of Data in Table 6-1 to

Obtain Cumulative Distribution Function of Matrix Porosities . . . . . . . . . . . . . . . . Att.A-33 


\subsection{Introduction}

\section{$1.1 \quad$ Purpose and Scope}

The purpose of this parameter description is to provide guidance on transferring porosity data obtained from outside of CAUs of interest at the NTS to the UGTA Project so that the data can be used to parameterize porosity in flow and radionuclide transport models. These models will be used to establish CAU contaminant boundaries, which will roughly correspond to perimeters within which future access to groundwater will be controlled owing to potential radioactive contamination originating from nuclear tests. There are several types of porosity described in this parameter description.

This attachment addresses the following data transferability factors and considerations mentioned in the main body of this document:

1. Parameter characteristics, including underlying dependencies on material properties or other parameters

2. Similarity of donor and receptor HSUs, with respect to geologic setting and other relevant characteristics for the parameter of concern

3. Type of measurement and/or interpretative technique, including measurement scale

4. Quality of documentation of measurements and interpretive methods

5. Considerations in assigning relative weights to different parameter datasets

This attachment does not directly address the following data transferability factors and considerations that are discussed in the main body of the document, although all of these factors are discussed briefly as they relate to the above-listed factors:

- Modeling approaches, including conceptual models and model scale,

- Heterogeneity

- Ranges in values

- Sensitivity of contaminant boundaries to parameter values

\section{$1.2 \quad$ Definitions}

Before the transferability of porosity data can be effectively discussed, different types of porosity must be defined. For the purposes of this attachment, six 
different types of porosity are considered for saturated geologic media: (1) total porosity, (2) effective flow porosity, (3) matrix porosity, (4) transport storage porosity, (5) disconnected storage porosity, and (6) fracture porosity. In defining these porosities, it is convenient to introduce the concept of a representative elementary volume (REV), which is a volume element of the domain of interest that is considered representative of the domain. In a porous medium domain, a $\mathrm{REV}$ is used for averaging quantities for which only volumetric averages are meaningful. The REV concept is used extensively in the porosity definitions that follow. However, starting in Section 1.0 and for the remainder of this attachment, the term REV is dropped in favor of using the less rigorous term "flow system," or simply "system." The definitions of the different types of porosity are:

\section{Total Porosity}

Void volume (not occupied by rock) in a REV divided by the total volume of the REV.

\section{Effective Flow Porosity}

The specific discharge (volumetric flow rate divided by cross-sectional area) divided by the effective flow velocity within a REV. The concept of effective flow velocity is used in modeling and experimental work to obtain a volumetrically averaged flow velocity that approximates the behavior of more complex and smaller-scale flow or transport processes within a REV. A good working definition of effective flow velocity is the travel distance across a REV divided by the mean travel time for a hypothetical ideal solute (nonreactive, nondiffusing, and having density equal to groundwater) to travel through the REV. This definition, as stated, is dependent on the method of measurement of mean solute travel time, so it is necessary to specify the appropriate method. Ideally, the solute should be injected uniformly as a pulse across a surface perpendicular to the pressure gradient, and its mass flux into the REV should be distributed according to the distribution of volumetric flow rates into the REV. Also, the solute response should be measured at a downgradient surface perpendicular to the pressure gradient, and it should be measured as a flux-averaged or "mixing cup" concentration

where

$$
\mathrm{C}_{\text {mixing cup }}=\frac{\sum_{\mathrm{i}} \mathrm{C}_{\mathrm{i}} \mathrm{Q}_{\mathrm{i}}}{\sum_{i} \mathrm{Q}_{\mathrm{i}}}
$$

$\mathrm{C}_{\mathrm{i}}=$ concentration in flow segment $\mathrm{i}\left(\operatorname{mass} / \mathrm{L}^{3}\right)$,

$\mathrm{Q}_{\mathrm{i}} \quad=$ volumetric flow rate in flow segment $\mathrm{i}\left(\mathrm{L}^{3} /\right.$ time $)$, and

$\sum_{i} Q_{i}=$ total volumetric flow rate through the REV).

The mean travel time should be calculated for the tracer response through a significant portion of the tail of the breakthrough curve but not for any extended tailing. "Extended tailing" is a subjective term, but a good working definition is the late portion of the tail of a breakthrough curve that is not fitted well by the advection-dispersion equation with parameters adjusted to achieve a good fit to the earlier portions of the breakthrough curve. The rationale for excluding extended 
tailing from the mean travel time calculation is that any fraction of the solute mass that moves through pathways of extremely long travel times will drastically increase the estimate of the mean, resulting in unrealistic and nonconservative estimates of effective flow porosity. Any solute mass fraction contributing to extended tailing or even to subsequent peaks that occur long after the initial peak(s) should be considered to be moving through transport storage porosity (see below). Note that in the case of real solutes that have finite diffusion coefficients (as opposed to hypothetical ones that do not diffuse), the effects of diffusion into stagnant water should be backed out of the breakthrough curves to obtain the response of a hypothetical nondiffusing solute.

\section{Matrix Porosity}

In consolidated fractured media, the matrix porosity is the void volume of the unfractured rock in the REV divided by the total volume of unfractured rock in the REV. In unconsolidated porous media (e.g., alluvium, valley fill deposits), the matrix porosity is the internal void volume (not occupied by rock) within the consolidated material in the REV (i.e., grains or cobbles of the media) divided by the total volume of the grains or cobbles of the media. Note that this definition of matrix porosity in unconsolidated porous media is a departure from the common practice of equating matrix porosity with total interconnected porosity (effective flow porosity plus transport storage porosity) in such media. Thus, when methods of measuring effective flow porosity plus transport storage porosity in unconsolidated media are discussed later in this attachment, many readers will consider these to be measurements of matrix porosity (sometimes also called "primary" porosity in unconsolidated media).

\section{Transport Storage Porosity}

Transport storage porosity is the volume of nonflowing water in the REV that is interconnected by diffusive pathways to the effective flow porosity in the REV divided by the total volume of the REV. "Nonflowing" water is considered to be any water in the REV that is not part of the effective flow porosity. Transport storage porosity may include water that is not considered part of matrix porosity (e.g., nearly stagnant water in dead-end or poorly-connected fractures in fractured media, or very slow moving water in clay lenses or fine-grained sediments in unconsolidated porous media). An alternative definition of transport storage porosity is the fraction of total porosity that does not advect fluids but remains interconnected by diffusive pathways to void space capable of advecting fluids.

\section{Disconnected Storage Porosity}

Disconnected storage porosity is the volume of nonflowing water in the REV that is not connected by diffusive pathways to the effective flow porosity divided by the total volume of the REV. An alternative definition is the fraction of total porosity that neither advects fluids nor diffuses fluids into void space capable of advection.

\section{Fracture Porosity}

Fracture porosity is attributed to void space created by fractures in consolidated media. Fracture porosity should not be confused with effective flow porosity, as 
there can be fractures that contain stagnant water (transport storage porosity), and even fractures that are not connected by diffusion pathways to effective flow porosity (disconnected storage porosity). Some fractures may be filled with precipitates, colloids, or alteration products, resulting in less void space (a lower individual fracture porosity) than an unfilled fracture. Fractures that become "sealed" to the point where their permeability is less than or equal to the surrounding matrix should be considered part of matrix porosity.

\subsection{Relationships Between the Different Types of Porosity}

Figure 1-1 and Figure 1-2 are two-dimensional (2-D) schematic depictions of the different types of porosity in fractured rock and unconsolidated porous media, respectively. The rectangular domain in each figure is a 2-D representation of the entire rock mass, with white representing solid rock. The total porosity is the colored area divided by the total area of the rectangle. The effective flow porosity is the blue area divided by the total area of the rectangle. The transport storage porosity is the red area (including both solid red and the red dots) divided by the total area of the rectangle, and the disconnected storage porosity is the green area divided by the total area of the rectangle. Matrix porosity in Figure 1-1 is the sum of the red and green areas excluding the solid red areas that correspond to dead-end fractures divided by the total area of the rectangle. Matrix porosity in Figure 1-2 is the sum of the lightly-hatched red and green areas within the oval-shaped grains (in the blow-ups) divided by the total area of all the oval-shaped grains.

Effective flow porosity, transport storage porosity, and disconnected storage porosity are conceptualized as mutually exclusive subsets of total porosity in Figure 1-3. Matrix and fracture porosity, however, are not necessarily mutually exclusive of any of the other types of porosity.

It should be noted that the designations of the different types of porosity in Figure 1-1 and Figure 1-2 are dependent on the REV that is selected from within a given flow system, and the designations are especially sensitive to the scale of the REV. For instance, if a small REV were selected from within the red dotted region (transport storage porosity) of Figure 1-1, then some of what was transport storage porosity at the larger scale would become effective flow porosity at the smaller scale. In essence, some portion of any REV will always be effective flow porosity. In this attachment, we are concerned primarily with very large REVs that may constitute a significant portion of a CAU or even an entire CAU.

\subsection{How Each Type of Porosity Affects Contaminant Transport}

The different types of porosity play different roles in contaminant transport through saturated geologic media. These roles are briefly summarized here.

\section{Total Porosity}

Total porosity represents the maximum possible porosity that contaminants can experience as they migrate through a REV. The total porosity is useful for calculating an effective upper bound of the amount of radionuclide dilution that 


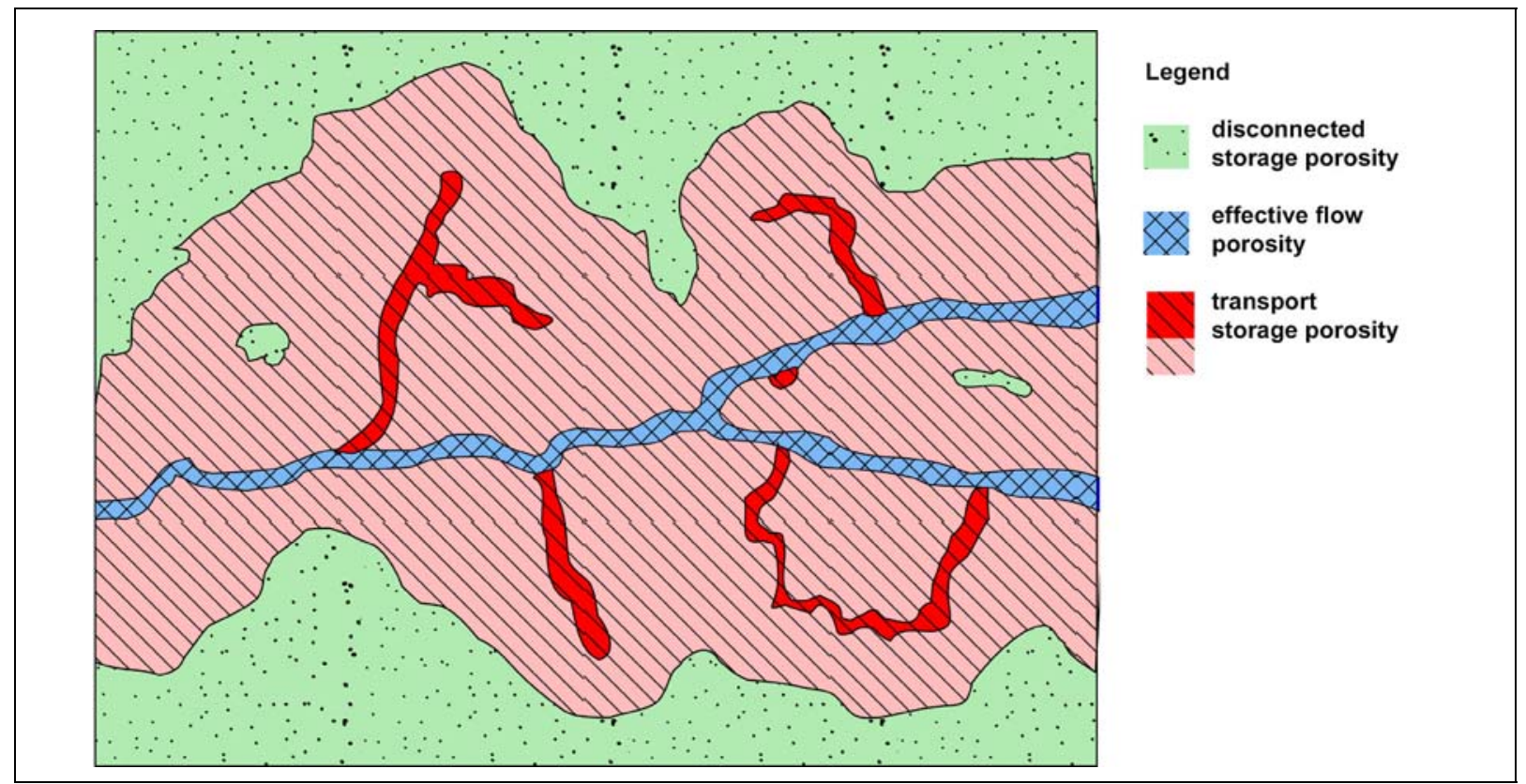

Figure 1-1

Depiction of the different types of porosity in a fractured rock. Blue is effective flow porosity, red is transport storage porosity, and green is disconnected storage porosity. Although it is shown to be the case here, effective flow porosity in fractured rock is not necessarily confined to fractures.

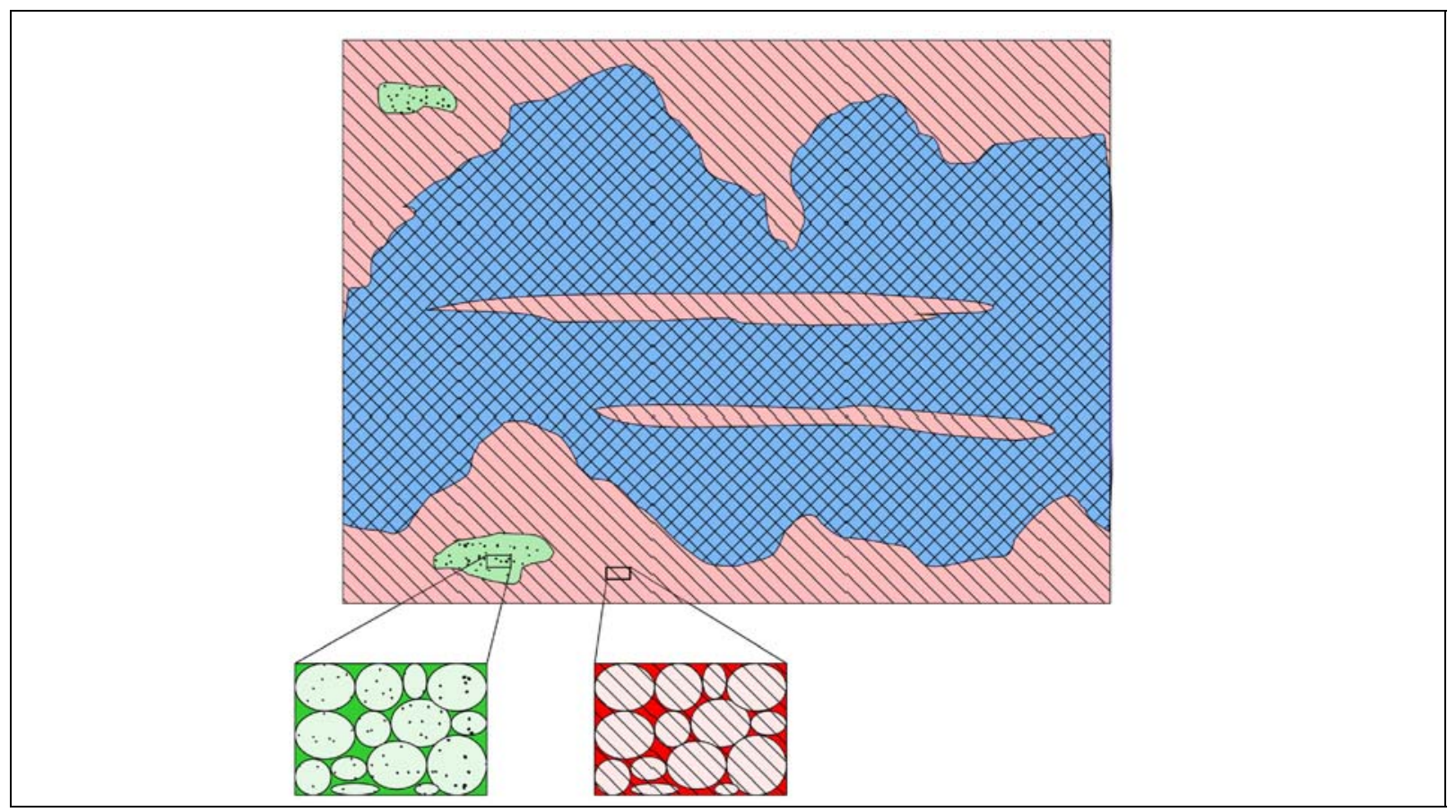

Figure 1-2

Depiction of the different types of porosity in unconsolidated porous media. Blue is effective flow porosity, red is transport storage porosity, and green is disconnected storage porosity. Note that this depiction is not intended to preclude any spatial distribution of transport storage porosity and disconnected storage porosity in unconsolidated media. 


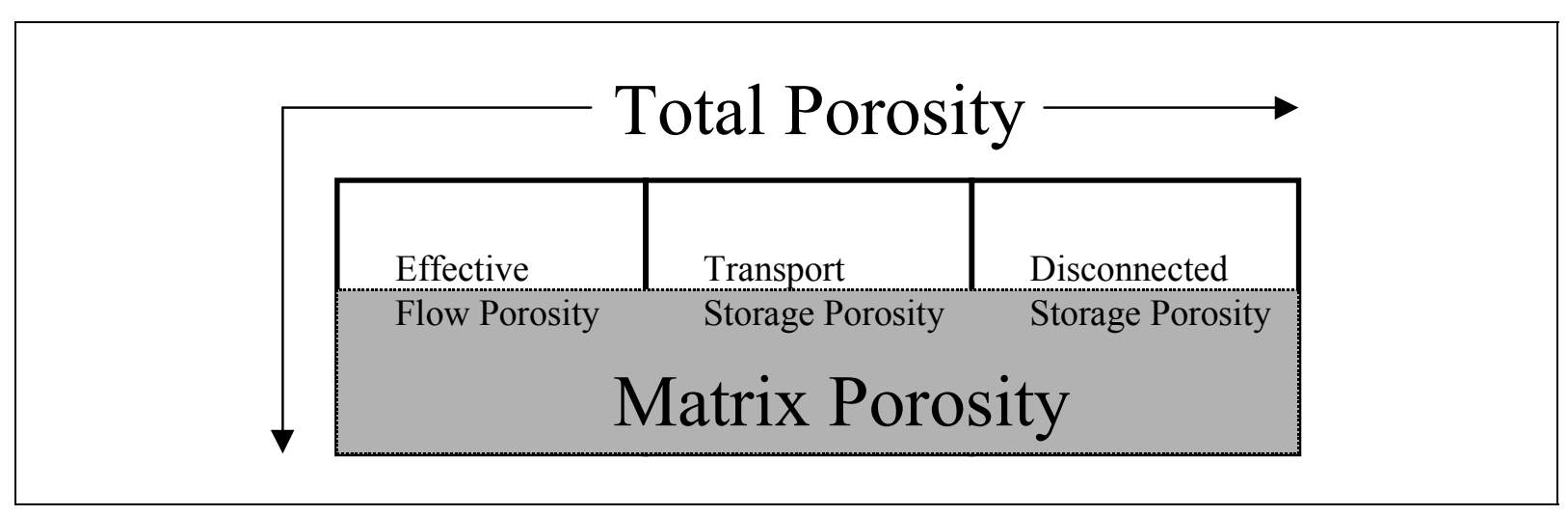

Figure $1-3$
Relationships between the different types of porosities. The gray area is matrix porosity, and the
white area is porosity that is outside the matrix. Note that the relative volumes of regions are not
intended to represent any particular type of flow system or geologic media.

can occur in a flow system or a lower bound of groundwater travel times given a specific discharge estimate. However, both of these estimates are of limited use because they are nonconservative for radionuclide transport. It is very possible that only a fraction of the total porosity of a REV will be accessible to radionuclides (except perhaps over very large time scales) because of diffusion limitations and physical boundaries that could isolate portions of the total REV porosity.

\section{Effective Flow Porosity}

The effective flow porosity governs groundwater and radionuclide travel times through a REV. The specific discharge divided by the effective flow porosity yields an estimate of the mean groundwater velocity through a REV, and it results in a conservative estimate of the mean travel time of nonsorbing radionuclides. Actual mean travel times could be greater if radionuclides can diffuse into nonflowing porosity that is connected to the flowing porosity.

\section{Matrix Porosity}

Matrix porosity is a subset of the total porosity that is defined by its location and physical attributes in the REV rather than by groundwater flow velocities or diffusion accessibility. As such, matrix porosity can consist of effective flow porosity, transport storage porosity, and disconnected storage porosity all within the same REV. In fractured rock with significant permeability contrast between fractures and matrix, the matrix porosity is generally taken to be a reasonable first-cut estimate of transport storage porosity (which assumes that the volume of unfractured rock in the REV is approximately equal to the total volume of the REV). However, diffusion-limited transport of solutes makes it likely that, over short time scales, only those portions of the matrix porosity relatively close to flowing fractures will see significant concentrations of solute. In alluvium or valley-fill settings, matrix porosity (porosity within consolidated materials in the REV; i.e., cobbles and grains) is very likely to function as transport storage porosity and disconnected storage porosity. In cases of large permeability contrasts in the extragranular porosity of an unconsolidated REV, matrix porosity 
could be a rather small subset of the transport storage porosity. In both fractured and porous media, the role of matrix porosity is to provide a reservoir of stagnant or nearly-stagnant water for radionuclides to diffuse into (from the effective flow porosity), thus delaying their movement through the flow system.

\section{Transport Storage Porosity}

The transport storage porosity serves to delay radionuclide transport through a flow system by providing a reservoir of stagnant or nearly-stagnant water for radionuclides to diffuse into (from the effective flow porosity). Transport storage porosity may also provide a great deal of surface area for radionuclide sorption, which will cause further delay in the transport of sorbing radionuclides. The greater the ratio of transport storage porosity to effective flow porosity, the greater the radionuclide transport delay. For nonsorbing radionuclides over long time and distance scales, an upper bound for the effective retardation factor associated with diffusive mass transfer between effective flow porosity and transport storage porosity is 1 plus the ratio of transport storage porosity to effective flow porosity (see Matrix Diffusion Attachment B for more discussion).

\section{Disconnected Storage Porosity}

The disconnected storage porosity plays no role in radionuclide transport through a flow system. Filling this porosity with solid rock would not change flow or transport through the REV. However, the geometric configuration of this porosity, if amenable to characterization and if different from one REV to another, can significantly change the flow patterns and consequently the contaminant migration pathways within different REVs.

\section{Fracture Porosity}

In fractured consolidated rock, it is frequently the case that the vast majority of the effective flow porosity in the REV is a subset of fracture porosity. This is especially true when the permeability of the unfractured matrix is low. Because effective flow porosity is of much greater practical importance for CAU-scale modeling (and it is more easily measured), fracture porosity is not specifically addressed in the remainder of this attachment. 


\subsection{Porosity Measurements}

Porosity measurements are discussed in this section so that the reader will have a better understanding of some of the methods and uncertainties associated with the methods of measurement. The intent is to help the reader make better-informed decisions to accept or reject porosity data for the UGTA project and also to provide background information to help in implementing the weight and multiplier system discussed later in this attachment.

\section{$2.1 \quad$ Laboratory Porosity Measurements}

Laboratory measurements are best suited for obtaining estimates of matrix porosity. Measurements of other types of porosity generally require measurement scales larger than are practical in the laboratory.

\subsubsection{Matrix Porosity in Consolidated Media}

Laboratory measurements of matrix porosity in consolidated rock samples are relatively straightforward. The most common method is to:

1. Weigh an oven-dried sample of the material cut into a convenient shape and size. Bigger is better for representativeness.

2. Saturate the sample with water under vacuum until its weight no longer increases with successive measurements. Saturation may take a significant time for low porosity and low permeability samples.

3. Determine the volume or weight of water displaced by the saturated sample. Alternatively, if the sample is large enough and cut to a convenient shape, the volume can simply be calculated.

4. Matrix porosity, $\phi_{\mathrm{m}}$, is calculated from the following equation:

$$
\phi_{\mathrm{m}}=\frac{\left(\mathrm{W}_{\mathrm{s}}-\mathrm{W}_{\mathrm{d}}\right)}{\gamma_{\mathrm{w}} \mathrm{V}}
$$

where

$$
\begin{aligned}
\mathrm{W}_{\mathrm{s}} & =\text { weight of saturated sample, } \mathrm{F} \text { (force) } \\
\mathrm{W}_{\mathrm{d}} & =\text { weight of dry sample, } \mathrm{F} \\
\gamma_{\mathrm{w}} & =\text { specific weight of water at temperature of measurement, } \mathrm{F} / \mathrm{L}^{3} \text {, and } \\
\mathrm{V} & =\text { volume of sample, } \mathrm{L}^{3} .
\end{aligned}
$$


Variations of this approach include using another liquid besides water (should be a wetting fluid to ensure entry into small pores), and placing the sample under a carbon dioxide $\left(\mathrm{CO}_{2}\right)$ atmosphere prior to saturation (because $\mathrm{CO}_{2}$ will dissolve in water more readily than air, resulting in more rapid and complete saturation).

Probably the biggest experimental uncertainties associated with these measurements are incomplete sample saturation and errors associated with water draining from very large pores at or near the surfaces of saturated samples when they are weighed. It is often best to simply not count such large pores as contributing to the volume of the sample. Incomplete sample saturation can occur when some of the internal porosity is very poorly connected or not connected at all with the pores on the exterior of the sample. In this case, the matrix porosity will be underestimated, although the porosity that is missed will likely act as disconnected storage porosity. Errors associated with water displacement measurements can also be significant with small samples. If sufficient material is available, it is recommended that measurements be made on several samples so that statistical variability in both samples and measurements can be evaluated.

Another laboratory method of matrix porosity measurement is mercury porosimetry (Leon y Leon, 1998). In this method, the amount of liquid mercury injected into a sample is measured as a function of mercury pressure applied. The method was originally developed to obtain pore-size distributions, but porosity is also readily obtained. The method is considerably more expensive than the water saturation method described above, and it involves both specialized equipment and the use of a toxic heavy metal, so it is usually justified only when pore-size distributions are desired. Gas adsorption methods can also be used to obtain estimates of porosity and pore-size distributions (Lee et al., 1996).

\subsubsection{Matrix Porosity in Unconsolidated Media}

Although the same measurement methods can be used for unconsolidated media as for consolidated media, it is generally more difficult to estimate matrix porosity in unconsolidated media (e.g., alluvium, valley fill material). Grains and cobbles in unconsolidated media can range in size from microns to meters, and they can consist of many different types of rock, depending on the rock sources contributing to the depositional sequence. As a result, measurements can be highly dependent on sample size and sampling location. Furthermore, sampling tends to "disturb" unconsolidated media, generally resulting in higher intergranular porosities after sampling than prior to sampling (i.e., in situ). It is very difficult to reproduce in situ conditions by repacking or otherwise manipulating the material after it has been sampled. Thus, it can be a significant challenge to achieve representativeness in matrix porosity measurements of unconsolidated samples.

Besides problems of representativeness, there are inherent difficulties in measuring the matrix porosity of geologic materials containing fine-grained sediments. When trying to compare dry and saturated weights, for instance, fine-grained sediments can retain a considerable amount of extragranular moisture that is technically not within the grain matrix. It may be necessary to use centrifugation to force water to drain from the interstitial spaces of such 
sediments. However, some extragranular moisture is still likely to be retained after centrifugation, and some intergranular moisture is likely to be drained, leading to errors in matrix porosity measurements. Some sediments, such as clays, may also swell when they are saturated, resulting in different saturated and unsaturated volumes, and hence porosities. Also, clays may clump together when dry (e.g., clumps in cuttings), giving the appearance of loosely-consolidated large-grained sediments when in fact they are unconsolidated fine-grained sediments when saturated. Failure to recognize this situation may lead to false inclusion of extragranular porosity in matrix porosity measurements. All of these difficulties increase the uncertainty associated with laboratory matrix porosity measurements of unconsolidated media. However, the biggest uncertainty in most systems is probably the degree of representativeness of the sample(s).

\subsubsection{Laboratory Measurements of Other Types of Porosity}

Laboratory measurements of effective flow porosity are not really possible in a laboratory setting because the scales of measurement are never large enough to capture the flow heterogeneity that occurs in the field. This statement is true for both consolidated and unconsolidated media. In unconsolidated media there is the additional complication that repacking of sediments in columns or sandboxes is invariably nonrepresentative because it is impossible to replicate fine-scale layering and structure found in the field. This nonrepresentativeness leads to inaccurate estimates not only of effective flow porosity, but also total porosity and transport storage porosity in unconsolidated media. Even when the general appearance of the field setting is captured, it is common to have much higher porosities and permeabilities in the laboratory because the reworked grains tend to pack in random orientations, leaving larger pore throats than are typically found in field sediments. Also, the lithostatic confining pressures at depth are often not reproduced in the laboratory, which can lead to artificially high laboratory porosities. This same problem applies to obtaining estimates of effective flow porosity in fractured rocks, as fracture apertures can become artificially large when lithostatic pressures are removed, or conversely, they can become artificially small if shear-induced offsets of fracture surfaces in the field are not reproduced in the laboratory (Durham and Bonner, 1994).

In consolidated media, matrix porosity often provides a good estimate of total porosity because fracture porosities tend to be quite small. Exceptions can occur in karstic systems that have large interconnected void spaces as well as in hard crystalline rocks where matrix porosity is exceedingly low. Matrix porosity is often used as a good estimate of transport storage porosity in consolidated fractured media. However, the analyst must be aware that (1) part of the matrix may also function as or contribute to effective flow porosity and (2) the matrix pores may not be sufficiently interconnected that solute diffusion can occur throughout the entire matrix. In both cases, matrix porosity estimates will overestimate transport storage porosity.

In general, it is recommended that effective flow porosity and transport storage porosity not be estimated directly from laboratory measurements. Also, total porosity in unconsolidated media should not be estimated from laboratory measurements. Thus, when considering the transfer of effective flow porosity 
data, transport storage porosity data, and total porosity data in unconsolidated media, laboratory measurements should be accepted only with considerable caution and should probably not be exclusively relied upon in the development of parameter distributions.

\section{$2.2 \quad$ Field Porosity Measurements}

Field measurements of porosity are generally inferred measurements of either total porosity or effective flow porosity. However, as mentioned above, total porosity measurements are generally good estimates of matrix porosity in consolidated media provided the measurements are not made in karstic systems with large interconnected void spaces. The following two sections briefly describe methods of measuring total and effective flow porosity in the field using geophysical logs and hydraulic/tracer methods, respectively.

\subsubsection{Geophysical Logs}

Borehole geophysical logs do not measure porosity directly. However, total porosity can be inferred from gamma-gamma, neutron, acoustic velocity logs, and borehole gravimetry logs. Additionally, resistivity logs can provide a measure of interconnected porosity (sum of effective flow porosity and transport storage porosity) within a formation. Nuclear magnetic resonance (NMR) logs can provide measures of "free" versus "bound" water in formations, with the "free" water generally being considered to be an indicator of effective flow porosity. The details of these logging techniques (what they specifically measure, equipment and how it is deployed, calibration methods) are not provided here; rather, the reader is referred to Keys (1997), A Practical Guide to Borehole Geophysics in Environmental Investigations and references provided therein. Keys (1997) addresses all of the above-mentioned logging techniques except for borehole gravimetry and NMR. Brief summaries of the measurements and how porosity is inferred from them are provided here.

\section{Gamma-Gamma Logs}

The gamma-gamma log actually measures rock bulk density, from which total porosity can be inferred from the following equation (Keys, 1997):

$$
\phi=\frac{\text { grain density - bulk density }}{\text { grain density - fluid density }}
$$

The method requires independent measurements of grain density and fluid density, as well as a properly calibrated probe to ensure accurate bulk density measurements.

\section{Neutron Logs}

Neutron logs effectively measure water content in formations, which can be translated to total porosity if the system is fully saturated. Again, the probe must be properly calibrated to ensure accurate measurements. The radius of investigation for both gamma-gamma and neutron logs are dependent on total porosity, but both are generally only a few inches to a foot or so beyond the borehole wall. Thus, both logs provide excellent vertical profiling of total 
porosity, but lateral profiling is limited to very near the borehole. Gamma-gamma logs have greater penetration in high porosity materials, and neutron logs have greater penetration in low porosity materials. Porosity data derived from these logs can be called into question if it is suspected that borehole drilling and completion altered the properties of the formation in the immediate vicinity of the borehole. Other factors that can affect these logs and should be carefully accounted for during calibration or in log interpretations are casing materials, muds or grouts used in completions, and water salinity. Also, neutron logs are effective for inferring total porosity only when systems are fully saturated; partial saturation leads to underestimation of porosity.

\section{Acoustic Velocity Logs}

Acoustic velocity logs can be used to infer total porosity from transit times of acoustic waves traveling between a source and multiple receivers (generally located on the same probe and detecting acoustic energy refracted from the formation). Total porosity, $\phi$, is inferred from the following equation (Keys, 1997):

$$
\phi=\frac{\text { transit time of } \log -\text { transit time of matrix }}{\text { transit time of fluid }- \text { transit time of matrix }}
$$

The transit time through the matrix (rock) and the water must be known to apply the method, but wave velocities are well established for most rocks and for "fresh" water and brines. The radius of investigation is 1 to 4 feet beyond the borehole wall, depending on the wavelength, rock type, and porosity. Lower porosities and consolidated hard rocks (e.g., granites, gabbros, dolomites) have greater effective penetration distances. Acoustic velocity logs work only in water-filled boreholes (to allow the acoustic energy to be efficiently transmitted to and from the formation), and they work best in uncased boreholes. Casing must be well-bonded to the formation rock for the method to work effectively. Acoustic velocity logs do not work well for inferring porosity in high-porosity unconsolidated systems. These logs are also known to "miss" the porosity in fractures and large voids, often resulting in lower total porosity estimates compared to gamma-gamma or neutron logs. However, in this regard, acoustic velocity logs may actually provide a better estimate of matrix porosity than the other logs.

\section{Borehole Gravimetry Logs}

Perhaps the best type of borehole geophysical log for indirectly measuring total porosity is the borehole gravimetry log (Herring, 1990). This log is not discussed in Keys' (1997), probably because it is expensive and not widely used. The method involves measuring acceleration due to gravity at various points or "stations" along a borehole, and it provides a direct measure of local bulk density, from which porosity is inferred (Equation A-2). The major advantage of the method is that the radius of investigation is significantly greater than other borehole geophysical logs, typically on the order of tens of meters. However, it has the disadvantage of being quite expensive compared to other geophysical logs because of the specialized equipment and requirements for local "calibration" of gravity measurements. Borehole gravimetry is a service that is currently offered by only one commercial company, EDCON Inc., of Denver, CO (www.edcon.com). 


\section{Resistivity Logs}

Borehole resistivity logs provide an indirect measure of interconnected porosity in a system. Referring to the porosity definitions of Section 1.2, these logs are sensitive to the effective flow porosity plus a majority of the transport storage porosity in a system, but not the disconnected storage porosity. Disconnected porosity is not "seen" because it does not provide a continuous pathway for conducting electrical current through the formation, which is the basis of the method. Borehole resistivity logs are generally used as a method of determining water quality, with water salinity being inferred after obtaining some knowledge of formation porosity by other means (generally from one of the logs mentioned above). However, if water quality is known from chemical analyses of water samples, then the log, in principle, can be used to infer porosity. The equation used to determine porosity from resistivity measurements is (Keys, 1997):

$$
\phi=\left(\frac{\mathrm{R}_{\mathrm{w}}}{\mathrm{R}_{\mathrm{t}}}\right)^{1 / \mathrm{m}}
$$

where

$$
\begin{array}{ll}
\mathrm{R}_{\mathrm{w}} & =\text { water resistivity, ohm- } \mathrm{L} \\
\mathrm{R}_{\mathrm{t}} & =\text { true formation resistivity, ohm- } \mathrm{L}, \text { and } \\
\mathrm{m} & =\text { cementation factor. }
\end{array}
$$

Unfortunately, the method is not straightforward, as the measurement of the formation "cementation factor", $m$, is generally conducted on core samples (although some general rules of thumb can be followed for different types of rock). Also, the calculation of true formation resistivity from direct field measurements is quite complicated. The radius of investigation of borehole resistivity $\log$ s is a function of probe configuration, but it is generally several feet beyond the borehole wall. In principle, transport storage porosity could be estimated from the porosity inferred from resistivity logs minus the effective flow porosity determined by other methods.

\section{Nuclear Magnetic Resonance Logs}

Nuclear magnetic resonance logging has become increasingly common in the petroleum industry and can have application in assessing effective porosity in groundwater hydrology. NMR logging uses static and pulsed radio-frequency (RF) magnetic fields to make downhole spin-echo magnetic resonance measurements. The basic principle of the NMR measurement is that a static magnetic field polarizes hydrogen protons found in the formation fluids. A RF magnetic field then rotates the protons $90^{\circ}$ to the transverse plane. A second pulse is applied after a specific time to rotate the protons $180^{\circ}$, causing a spin-echo at time TE. A series of $180^{\circ}$ pulses are then applied at equal time intervals, each pulse causing a spin-echo. Amplitude decay of the spin-echo train versus time is recorded to form the basic NMR dataset. Spin-echo amplitude is interpreted as a measure of the volume of the formation fluids. The fluids need not be connected via continuous interstitial pathways to the borehole to be "sensed." Amplitude decay time of the spin-echo train is designated as $\mathrm{T}_{2}$. Hydrogen protons present in the fluids found in large pore spaces relax at a slower rate than do hydrogen protons in smaller pores. Fluid in large pores contributes to the slower decaying 
portion of the spin-echo, (i.e., larger $\mathrm{T}_{2}$ values), while fluid in small pores produce the faster decaying portion of the spin-echo (smaller $\mathrm{T}_{2}$ values). The maximum spin-echo amplitude at $\mathrm{t}=0$ is taken as an indicator of the formation's "open" porosity.

The terminology common in the petroleum industry is total porosity and effective or "free fluid" porosity with the difference between the two being the volume of "bound" fluid. The distinction between effective porosity and bound fluid is somewhat arbitrary. This threshold is partially driven by instrument sensitivity but mainly a function of what fluid velocity is considered negligible. NMR logging is highly limited by the volume and depth of rock that the tools investigate (the radius of investigation is on the order of a few inches from the borehole wall). It can give useful estimates of effective porosity in unfractured rock matrices. Application in lithologies differing from those found in petroleum provinces may require additional calibration, especially when bound fluid may represent a significant portion of the total fluid.

\subsubsection{Hydraulic and Tracer Testing Methods}

Hydraulic and tracer methods for determining porosity in the field are typically more involved and more expensive than borehole geophysical methods (with the possible exception of borehole gravimetry).

\section{Borehole Flow Surveys}

The simplest type of hydraulic test is the borehole flow survey, which is often considered to be a "log" rather than a hydraulic test. Flow surveys provide information on the vertical distribution of flow within a borehole, from which qualitative information can be inferred about flow heterogeneity and, ultimately, effective flow porosity. Flow surveys can be conducted with impellers (or "spinners"), heat pulses, or tracer pulses. Impellers are generally better for higher flow rates induced by pumping, while heat and tracer pulses are more accurate for lower flow rates generally associated with ambient conditions. Impellers are usually "trolled" up and down a borehole either during pumping or under ambient conditions, with the best logs involving measurements at different trolling rates. Flow surveys should be conducted under both ambient and pumped conditions so that the flow induced by pumping can be obtained by difference. Temperature measurements are often made in conjunction with flow measurements; which can provide additional information on flow distribution in boreholes, as temperature inflections occur where there is an influx of warmer or cooler water.

It is quite common in consolidated fractured rocks for only a fraction of the formation to conduct a significant volumetric water flow. In extreme cases, only one or two narrow flow zones, usually associated with open fractures, contribute essentially all of the water produced from boreholes. A qualitative estimate of effective flow porosity can be obtained by simply dividing the sum of the lengths of the flowing intervals by the total length of the screened or open sections (saturated) in the borehole. 
Difficulties and ambiguities associated with inferring effective flow porosity from flow surveys include (1) flowing interval lengths are easily overestimated, (2) flowing intervals can be difficult to define when gravel packs and screens are part of well completions, and (3) vertical boreholes are biased toward interrogating horizontally-oriented flow features and against vertically-oriented features.

\section{Pumping or Slug Tests}

Cross-hole pumping tests in confined aquifers can, in principle, yield indirect and rough estimates of the total porosity in an aquifer based on storativity estimates, which are derived from the analysis of pressure-time responses. Aquifer storativity, S, is given by (Freeze and Cherry, 1979):

$$
\mathrm{S}=\rho \operatorname{gh}(\alpha+\phi \beta)
$$

where

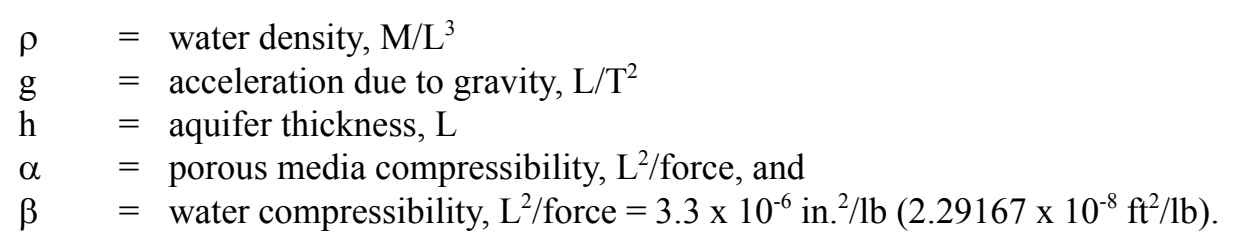

Slug tests (injecting water rather than pumping it from the aquifer) are often easier and cheaper to conduct than pump tests, and, in principle, they provide the same information. However, they are generally less desirable than pump tests because their duration is limited. Single-well pumping tests do not provide good estimates of storativity because pressure responses in pumped wells are highly insensitive to storativity.

Storativity is technically defined as the volume of water that an aquifer releases from "storage" per unit surface area of aquifer per unit decline in the component of hydraulic head normal to that surface (Freeze and Cherry, 1979). Unfortunately, $\alpha$ in equation A-5 is often poorly known and is frequently much larger than $\phi \beta$, so estimates of storativity are typically insensitive to $\phi$ and therefore yield poorly-constrained, and thus highly uncertain, estimates of total porosity. Storativities derived from pressure-time responses can also be different for different semi-analytical solutions used to "fit" the responses. The different semi-analytical solutions are dependent on various assumptions about the nature and extent of the aquifer (e.g., confined, unconfined, leaky-confined) which can be quite uncertain (although certain pressure-time response characteristics are generally associated with certain aquifer characteristics). All of these semi-analytical solutions assume radial flow or at least a homogeneous system, so storativities and porosity estimates will be in error if the flow system is heterogeneous, as it almost always is.

\section{Use of Barometric Efficiencies and Responses to Earth Tides}

A somewhat refined method of using aquifer storativity to estimate total porosity is to couple storativity measurements with barometric efficiency estimates. 
Barometric efficiency is defined as the ratio of the magnitude of an aquifer's pressure responses resulting from changes in surface barometric pressure (which have the opposite sign of barometric pressure changes) to the magnitude of the changes in barometric pressure. It is obtained by long-term monitoring of barometric pressure and aquifer head (under ambient conditions). Calculation of porosity in porous media is accomplished through the following a relationship derived for confined aquifers by Todd (1959):

$$
\phi=\frac{\mathrm{S}_{\mathrm{s}}(\mathrm{BE})}{\gamma \beta}
$$

where

$$
\begin{aligned}
& \mathrm{S}_{\mathrm{s}}=\text { specific storage }=\mathrm{S} / \mathrm{h}, \\
& \mathrm{BE}=\text { barometric efficiency, } \\
& \gamma \quad=\text { unit weight of water }=62.496 \mathrm{lb} / \mathrm{ft}^{3}, \text { and } \\
& \beta \quad=\text { water compressibility, } \mathrm{L}^{2} / \text { force }=3.3 \times 10^{-6} \mathrm{in}^{2} / \mathrm{lb}\left(2.29167 \times 10^{-8} \mathrm{ft}^{2} / \mathrm{lb}\right) \\
& \mathrm{h}=\text { aquifer thickness, } \mathrm{L}
\end{aligned}
$$

The quality of the porosity estimate from equation (A-7) is generally considered marginal, but it is should be better, in most cases, than that obtained from equation (A-6).

A related method of porosity estimation is that of analyzing hydraulic responses to earth tides (Morland and Donaldson, 1984). Estimates in this case should correspond to interconnected porosity (effective flow porosity plus transport storage porosity), and they are quite sensitive to porous media compressibility and other properties that may be highly uncertain or variable in aquifers. Thus, the method is not widely accepted and should be treated as having considerable uncertainty.

\section{Tracer Tests}

Probably the best method for obtaining effective flow porosity estimates at the field scale is cross-hole tracer testing. Effective flow porosity from a forced-gradient cross-hole tracer test is estimated using the following equation (Reimus, 2003):

$$
\eta=\frac{\mathrm{Q}_{\tau}}{\pi \mathrm{R}^{2} \mathrm{~h}}
$$

where

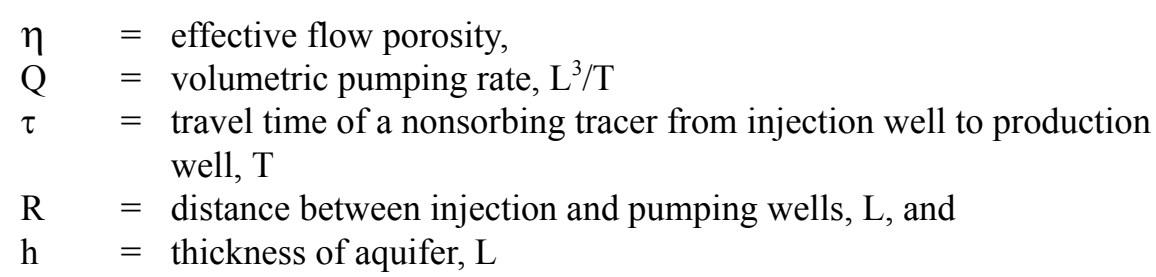

The tracer travel time in equation (A-7) should technically be the mean tracer arrival time at the production well, but first and peak arrival times are sometimes used, often with corrections to adjust them to be closer to a mean arrival time. 
Equation (A-7) assumes radial flow in a homogeneous, isotropic, confined aquifer. Effective flow porosity estimates obtained using this equation may be in error if there is considerable flow heterogeneity in the aquifer or if the aquifer is not confined. For instance, if flow to the production well is primarily from portions of the aquifer that do not pass close to the injection well or if flow pathways are highly tortuous, tracer travel times may be much longer than they would be under radial flow conditions, resulting in high effective flow porosity estimates. Partial recirculation of produced water into the tracer injection well can help reduce these uncertainties by forcing tracer mass away from the injection well into more permeable flow pathways that intersect the production well (Reimus, 2003). However, the errors and uncertainties can only be reduced, not eliminated.

Effective flow porosity estimates can also be obtained from single-well injection-withdrawal tracer tests, but these estimates are poorly constrained, and it is generally accepted that estimates from cross-hole tracer tests are far superior.

\section{Use of Ground Penetrating Radar}

One method that could potentially be used in conjunction with cross-hole tracer tests to obtain better estimates of effective flow porosity is ground-penetrating radar (GPR) (Liu et al., 1998). GPR provides 2-D images of salinity contrasts in saturated flow systems. The plane that runs through the two wells in which the source and receiver probes are emplaced is the "image plane." If two such wells were oriented perpendicular to an injection and production well in a cross-hole tracer test such that all the wells form a cross configuration $(+)$, GPR could potentially determine the cross-sectional area of tracer solution traveling through the plane of the two observation wells (between the injection and production well). Knowledge of this cross-sectional area could help in making qualitative corrections to the effective flow porosity estimate obtained from equation (A-7). For example, if the porosity determined from equation (A-7) is very large, but the cross-sectional area of the tracer plume in the image plane is small, then equation (A-7) is very likely overestimating flow porosity. This situation could occur if most of the flow to the production well is coming from a direction other than the direction of the injection well, resulting in long tracer arrival times at the production well. The information obtained from the GPR images could be used to adjust the porosity estimate downward accordingly. Tracer arrival time(s) at the image plane relative to arrival times at the production well could also be used to obtain refined quantitative estimates of effective flow porosity. For instance, the travel time from the image plane to the production well could be used to obtain an independent estimate of effective flow porosity that is not influenced by media disturbances caused by drilling/completion of the injection well, or by nonidealities associated with the injection procedure. By the time tracer solution makes it to the image plane, it should be migrating primarily in conductive flow pathways in the system, not artificial pathways caused by the injection procedure or as a result of the injection well being located in a low-permeability region of the aquifer. GPR requires boreholes that have no casing or nonmetallic casing. It has been used with some success in unconsolidated media where effective flow porosities are high, but it is more difficult to use in consolidated media with lower flow porosities. 


\section{Transport Storage Porosity from Tracer Tests}

Tracer tests can, in principle, provide estimates of transport storage porosity in both consolidated and unconsolidated media. To estimate transport storage porosity, it is necessary to simultaneously inject multiple nonsorbing solute tracers with different diffusion coefficients so that diffusion into transport storage porosity can be distinguished from dispersion (both processes tend to result in spreading, attenuation, and long tails in tracer breakthrough curves). Another less practical approach is to conduct multiple tracer tests at different flow rates through a system using the same nonsorbing tracer, or tracers with the same diffusion coefficients, in each test. Differences in the responses of the different tracers in the same test or the same tracer in tests at different flow rates can be used to obtain estimates of diffusive mass transfer rates as well as diffusion distances into the transport storage porosity. However, to get a good estimate of transport storage porosity, it is necessary for the tracers to diffuse throughout all of the accessible storage porosity, which could take a much longer time than is practical for most single-well or forced-gradient cross-hole tracer tests. Thus, estimates of transport storage porosity are probably best obtained from long-duration natural-gradient tracer tests with a sufficient number of monitoring/observation wells to define tracer migration. Also, the distributions of environmental tracers in flow systems, if they can be measured, might provide good indications of transport storage porosity. 


\subsection{Considerations for Data Transferability}

When evaluating porosity data for transferability to a NTS CAU, several factors must be considered. These factors are discussed in this section.

The geologic setting is probably the most important factor in considering the transferability of porosity data. If the geologic setting is significantly different from the CAU of interest, then the porosity data should be transferred only with caution, and in most cases, it should not be transferred at all.

The type of rock and its origin are the first factors that should be considered. Although exact matches between geologic settings, and especially rock origin, are highly unlikely, the rock type from which the porosity data is to be transferred should at least be the same as the potential receptor unit in the CAU of interest. Rock types can be characterized in many ways, but for the purposes of NTS CAUs, they should at least include the following categories:

- Metamorphic - Rocks formed as a result of high pressure and high temperature transformations (without melting) of previously deposited rocks. Metamorphosis typically occurs deep in the earth's subsurface, and the rocks are brought close to the surface by erosion or tectonic processes.

- Igneous, Intrusive - Crystalline rocks formed by cooling of melted rock beneath the earth's surface. Granite intrusions serve as an example.

- Igneous, Extrusive - Volcanic tuffs and lavas originating from volcanic ash flows and lava flows.

- Non-volcanic sedimentary rocks - Rocks formed by past deposition of inorganic or organic matter that has long since been consolidated by subsurface processes (e.g., pressure, geochemical and hydrothermal alteration). Limestone (carbonate) and sandstone serve as good examples.

- Alluvium/valley fill deposits - Unconsolidated material deposited in basins or low-lying areas by surface erosion processes. Alluvium can be formed by erosion of any of the above rock types.

Porosity data, as a general rule, should not be transferred between these different rock type categories. Data also should not be transferred between significantly 
different types of non-volcanic sedimentary rocks. For instance, sandstone porosities should not be transferred to a carbonate limestone setting.

Although similarity of rock types, as described above, is an overriding consideration in data transferability, there are additional geologic factors that must be considered. These factors can be thought of as lower-tier measures of similarity between different rocks of the same general "type." For instance, rocks that are volcanic in origin can have a wide range of characteristics depending on their depositional and cooling history as well as their geochemical and hydrothermal alteration history. Low viscosity lavas that cool quickly form glassy or vitric basaltic flows, while lavas that cool more slowly typically "devitrify," to form crystalline matrices. In general, porosity data obtained from glassy basalts should not be transferred to settings that contain primarily devitrified lavas (and vice-versa). Gases in lava flows can be trapped to varying degrees depending on flow viscosity, thus forming bubbles, which later become pores. These pores can vary from large isolated "vugs" to smaller, well-connected "bubbles" that form pumices. Ashflow tuffs can vary significantly in degree of welding, or how well their grains are "stuck together", which generally translates to varying degrees of intergranular porosity. Ash deep in the interior of a pyroclastic flow is likely to be more strongly welded than ash near the flow extremities because it stays at higher temperature for longer. Lavas and tuffs also can vary significantly in their alteration history. Under the right conditions, involving interaction with water at various temperatures and pressures over long periods of time, both types of volcanic rocks can become highly altered such that they contain significant amounts of clays and zeolites, or possibly other alteration products. These alterations can have dramatic effects on all types of porosity in the rocks.

Metamorphic and sedimentary rocks may also have different alteration histories that must be considered when transferring porosity data. Characteristics of alluvium deposits can vary significantly depending on the source rock type(s) and the deposition mechanisms. Alluvium can vary from large cobbly materials deposited in riverbeds, to fine clays and silty materials deposited in lakebeds, to grains of various sizes deposited by wind or water. All of these materials can be present simultaneously in systems with different degrees of order or layering, depending on past climate history and surface deposition processes. Alluvium can also be altered by geochemical or hydrothermal processes. Alluvium that becomes consolidated can ultimately be considered a sedimentary rock, although weak cementation of grains (for instance, from calcite formation) is usually not considered a transformation to a consolidated rock type.

Structural alteration must also be considered for different rock types. Faulting and folding resulting from tectonic activity can dramatically alter porosity of all types of rocks. Rocks with higher total porosity and less welding can deform without fracturing in fault zones such that matrix porosity and saturated permeability decreases relative to the original formation (Wilson et al., submitted). However, more competent rocks with lower total porosity generally fracture under stress, resulting in much greater effective flow porosity but only minor changes in matrix porosity in fault zones. Alluvium can, in principle, be reworked (disordered) by tectonic processes. 
A second consideration in transferring porosity data from other locations to NTS CAUs is the method of measurement. As discussed in Section 2.0, different methods yield data with different uncertainties and scales of relevance. Ultimately, the representativeness of the measurements when used in flow and transport models over scales of several kilometers must be considered.

In general, field-scale measurements of porosity should be given more weight than laboratory-scale measurements because the measurements are done at much more relevant scales for CAU modeling. The volume of rock measured in a laboratory experiment is typically only a few cubic inches, whereas the volume of rock in field experiments can be hundreds of cubic meters. Thus, field experiments are much more likely to provide representative values of porosity.

However, the uncertainty and quality of measurements must also be considered. For instance, a field-scale measurement of total porosity obtained from a pump test using equation (A-5) will probably be highly uncertain; therefore, it may not be nearly as useful as a laboratory measurement. Although general criteria for transferability of data cannot be specified because every dataset is unique, the following rules of thumb apply given current measurement technologies:

- Carefully calibrated geophysical logs of the type mentioned in Section 2.2 (other than resistivity and NMR logs) generally provide good estimates of total porosity in both consolidated and unconsolidated media. Borehole geophysics data are obtained over much larger volumes of rock than laboratory measurements. Borehole gravimetry $\operatorname{logs}$, although expensive, probably provide the most representative total porosity estimates in consolidated rocks because of the very large volume of rock interrogated. The total porosity measured by most borehole geophysical logs is often considered to be a reasonably good measure of matrix porosity in consolidated media (there are exceptions, such as karstic limestones). Resistivity logs can provide estimates of the sum of effective flow porosity and transport storage porosity, but these are generally considered to be poorer estimates than the total porosity estimates obtained by other borehole geophysics methods. NMR logs provide information on the "openness" of the total porosity, distinguishing between "free" and "bound" water. These logs are probably best used to supplement other geophysical logs and porosity estimates derived from them should be considered quite uncertain.

- Laboratory measurements provide unreliable estimates of effective flow porosity and transport storage porosity because the measurements are conducted at scales that do not capture field-scale heterogeneity in these types of porosity and also because it is difficult to achieve representativeness of subsurface conditions in the laboratory, particularly with unconsolidated materials.

- Laboratory measurements provide good estimates of matrix porosity in consolidated rocks. They are more accurate than geophysical logs, but 
they are also less representative because of their smaller scale. Geophysical logs are often calibrated with the help of laboratory measurements. Matrix porosity estimates in unconsolidated rocks are more uncertain than in consolidated rocks because it can be a significant challenge to obtain representative samples of unconsolidated rocks.

- Estimates of total porosity obtained from storativity estimates derived from pump (or slug) tests should be carefully evaluated, and in many cases, they are too poorly constrained and too uncertain to transfer or use. Estimates of total porosity obtained using barometric efficiency estimates coupled with storativity estimates from pump tests are only marginally better.

- Estimates of the sum of effective flow porosity and transport storage porosity obtained by analyzing water level fluctuations due to earth tides are generally considered very uncertain.

- Cross-hole tracer tests provide the best estimates of effective flow porosity in consolidated and unconsolidated saturated media. However, these estimates can still have a great deal of uncertainty associated with them. Although it has been demonstrated only a few times, ground-penetrating radar used in conjunction with cross-hole tracer tests could help reduce this uncertainty.

- Flow and temperature logs can also provide useful information that can be translated into effective flow porosity. However, this information is obtained over much smaller scales than cross-hole tracer tests.

- Transport storage porosity is difficult to estimate in geologic media. If it is thought that good estimates of interconnected porosity are obtained from resistivity logs, then transport storage porosity can be estimated from "resistivity porosity" minus effective flow porosity, as determined by another method. The reliability and uncertainty of these estimates will be directly related to the reliability and uncertainty in the porosity estimates used in these calculations. Estimates of transport storage porosity obtained from long-duration multiple-tracer tests or from measuring the distribution of environmental tracers in a system are probably better than those obtained from the above calculations, although they are difficult and expensive.

\subsection{Data Reduction and Analysis Method}

Even when the best possible measurement method is used, the methods and quality of data reduction and analyses must be considered when transferring porosity data. Data reduction in this context is defined as the process of converting raw data to analyzed data, which typically involves corrections or adjustments based on standards or calibration measurements. Poor data reduction or poor interpretive methods (sometimes because of old vintage) can reduce the confidence in or increase the uncertainty of an otherwise sound dataset. In many cases, it may be possible to re-analyze the data using more modern or acceptable 
methods. However, when this is not possible, it may be necessary to place less emphasis on the dataset than if the data reduction and analyses methods were optimal.

\subsection{Quality of Documentation}

Quality of documentation is also a factor in determining the transferability of porosity data. Poor documentation should reduce the relative value placed on a given dataset, and in extreme cases, it might even disqualify a dataset from being transferred. The documentation quality factor should not be confused with the data reduction and analysis method factor. A high-quality dataset and interpretative analysis can sometimes be very poorly documented. However, it is often the case that a poor analysis is also poorly documented, and in some cases, it may be difficult to distinguish between a poor analysis and poor documentation. 


\section{Weight and Multiplier System}

The recommended approach for formalizing the process of transferring porosity data obtained from outside a given CAU and for determining the relative weight that should be assigned to the data is to use a weight and multiplier system. This approach is also recommended for datasets obtained within a CAU of interest because, even though all such data will come from a relevant geologic setting, some CAU-specific datasets should rightfully be assigned greater emphasis than others because of differences in measurement methods, data quality, and documentation quality. The weight and multiplier system is quite subjective, which is not particularly appealing from a scientific perspective, but it is consistent with the subjective nature of the data transferability process. The system and process ultimately rely heavily on the documentation of the rationale for weights and multipliers, which essentially provides the rationale for the transfer of data.

In the weight and multiplier system, weights are determined for datasets based on the relevance of the geologic setting to the CAU, and multipliers (factors that the total weight score is multiplied by to obtain an overall score) are assigned based on the measurement method, the quality of data reduction and analyses, and documentation quality. The overall score for a given dataset is the sum of the weights multiplied by the product of the multipliers. Datasets with poor geologic setting relevance, while they may still be transferred, are assigned lower weights so they ultimately "count less" in determining the final parameter distribution used in CAU-scale modeling. Likewise, datasets associated with measurement methods having large uncertainties or inappropriate scales, or datasets of low-quality or having poor documentation are assigned lower multipliers so that they count less in the final CAU-scale application.

The rationale for using both weights and multipliers is that the geologic setting relevance and the data quality are two separate criteria that are largely independent. Weights are used for geologic setting relevance because this is an overriding consideration in most cases. Datasets obtained from the best possible measurement method and having high-quality analyses and documentation should nevertheless be rejected if the geologic setting is inappropriate. In a system based entirely on weights, such datasets could potentially still be assigned a relatively high weight score even though they are not appropriate for the CAU of interest. Multipliers are assigned after weights are determined so that appropriate credit can be taken for the measurement method and data/documentation quality. Although datasets will seldom be rejected outright at this stage, very low multipliers can be assigned because of a highly uncertain measurement method or very poor documentation. The weights and multipliers have relative scales rather than absolute scales. For instance, if there are only two datasets being considered, then 
multipliers of 1.0 and 0.5 for these sets will have the same effect as multipliers of 0.2 and 0.1 , respectively (i.e., the first dataset has a multiplier twice that of the second set in both cases). These relative scales make it imperative that the rationale for weights and multipliers be clearly documented (see Section 5.0) so that if additional data are later considered for transfer, they can be scored in a manner consistent with the previously transferred data.

This approach of "graded" transferability recognizes that most transfer decisions will not be a simple "yes or no", but rather there will be varying degrees of relevance of datasets to a given CAU, as well as varying degrees of measurement and documentation quality. It also recognizes that, while the goal is to make objective decisions regarding data transferability, there are almost always subjective elements involved in these decisions.

Section 3.1 provided guidance for determining the relevance of geologic settings of datasets to NTS CAUs. Sections 3.2 through 3.4 provided guidance for ranking measurement methods and for determining data reduction and documentation quality. Sections 4.1 and 4.2 provide guidance for stepping through these considerations to assign weights and multipliers, respectively, to datasets. The guidance provided in these sections can be deviated from if there are justifiable reasons for doing so. However, such deviations should be documented in the rationale for the selection of weights and multipliers (see Section 5.0).

\subsection{Weights (Relevance of Geologic Setting)}

Note that the overall weight for a dataset is the sum of the weights for each of the three criteria below.

\section{Rock Type}

If the rock type (categories of types provided in Section 3.1) is different from the type in the CAU, then the data should not be considered for transferability unless there is some unusual circumstance. That is, an overall weight score of zero should be assigned so that regardless of the measurement method or data quality, the dataset is rejected. If the rock type is the same, then the analyst should proceed to step 2. However, the analyst may reject data at this point even if it is from the same rock type because of an obvious difference in geology. For instance, sandstone data should not be considered for limestone settings even though both rocks are nominally sedimentary. Also, glassy basalt data should not be considered for devitrified lava settings even though both rocks are volcanic lavas.

\section{Deposition and Alteration History}

Similarities and differences in deposition history and geochemical/hydrothermal alteration should be evaluated and weights assigned using a sliding scale of 0 to 0.7 , with 0.7 indicating a very similar deposition and alteration history. Any score near zero should prompt the analyst to question whether the data should even be considered for transferability. This step represents a second point where data could potentially be rejected, with the examples above applying here as well. However, once data makes it past this step, it should no longer be rejected outright; instead, it should be assigned a low weight if it has questionable 
relevance to the CAU. Further guidance on how to assign sub-weights within the 0 -to- 0.7 sliding scale is not provided because of the multiplicity and complexity of considerations involved, as well as the desire to give the analyst(s) flexibility for a wide range of potential situations. However, the burden is on the analyst to document the rationale for the assigned weight (Section 5.0). The analyst is encouraged to use the formal statistical methods of addressing similarity discussed in Section 3.1 (using attributes other than porosity) to help determine and document a score.

\section{Structural Setting and Mechanical Alteration}

Similarities and differences in structural setting and mechanical alteration should be evaluated and weights assigned using a sliding scale of 0 to 0.3 , with 0.3 indicating a very similar structural setting. In this case, a score near zero should not necessarily disqualify a dataset from consideration. Again, the analyst is encouraged to use the formal statistical methods of addressing similarity discussed in Section 3.1 (using attributes other than porosity) to help determine and document a score.

Note: If CAU-scale models are developed that include domains of different rock types, deposition/alteration histories, and/or structural settings, individual datasets can be considered for each model domain and weights can be assigned on a case-by-case basis for each domain. For instance, given the concept of multiple hydrostratigraphic units (HSUs) in flow and transport models, a particular dataset could be "transferred" to different HSUs, with a different weight assigned to each HSU. This same approach can be extended to situations were HSUs contain multiple rock types or multiple deposition/alteration histories; the data could be transferred with different weights assigned to different portions of the HSU. Also, if different structural features are distinguished in model domains (e.g., faulted and unfaulted regions), datasets could be weighted differently for these different regions.

\subsection{Multipliers (Measurement Method and Quality of Analyses and Documentation)}

Note that the overall multiplier for a dataset is the product of the multipliers for each of the three criteria listed below.

\section{Measurement Method}

Measurement method considerations include both the quality/ uncertainty of the method and the scale of the measurement. These considerations were discussed in Section 2.0. General guidelines on relative rankings of measurement methods were discussed in Section 3.2. The numerical values listed below are intended for guidance only, but their values are chosen to reflect the relative emphasis that should be placed on different types of measurements for estimating a given type of porosity for CAU-scale modeling (given current measurement technologies). Higher numbers reflect what is considered to be a "better" measurement. Any method not listed below for a given type of porosity should be considered on a case-by-case basis, or it should be assigned a zero multiplier because the method does not apply. 
- Matrix Porosity in Consolidated Media

- Borehole gravimetry $\operatorname{logs}-1.8$.

- Gamma-gamma, neutron, and acoustic-velocity logs - 1.4 if borehole gravimetry logs are considered; 1.8 if there are no borehole gravimetry logs.

- Laboratory measurements by saturated-minus-dry weights or mercury porosimetry -0.8 .

- Pump (or slug) tests using estimates of storativity and equation (5) -0.1 .

Note: All of the above methods except for laboratory measurements estimate total porosity, not matrix porosity. However, it is assumed that in most consolidated media, matrix porosity is approximately equal to total porosity. If this is not true for a given geologic setting, then weights should be adjusted downward accordingly.

- Matrix Porosity in Unconsolidated Media:

- Laboratory measurements on representative samples - 0.6.

- Total Porosity in Consolidated or Unconsolidated Media:

- Borehole gravimetry $\operatorname{logs}-1.8$.

- Gamma-gamma, neutron, and acoustic-velocity logs - 1.4 if borehole gravimetry logs are considered; 1.8 if there are no borehole gravimetry logs.

- Laboratory measurements -0.5 for consolidated media; 0.2 for unconsolidated media.

- Pump (or slug) test using estimates of storativity and equation (5) 0.1 .

- Pressure responses to barometric fluctuations (coupled with storativity estimates) -0.2 .

- Effective Flow Porosity in Consolidated Media:

- Forced-gradient cross-hole tracer tests - 1.3; 1.7 if coupled with ground-penetrating radar imaging.

- Estimates obtained from flow and temperature logs and supporting information -0.6 (but could be less if only part of the borehole is open to formation). 
- NMR logs (coupled with logs measuring total porosity) - 0.3.

- Analysis of pressure responses to earth tides -0.2 .

- Effective Flow Porosity in Unconsolidated Media:

- Forced-gradient cross-hole tracer tests - 1.4; 1.8 if coupled with ground-penetrating radar imaging.

- Estimates obtained from flow and temperature logs and supporting information -0.6 (but could be less if only part of the borehole is open to the formation).

- $\quad$ NMR logs (coupled with logs measuring total porosity) - 0.4.

- Analysis of pressure responses to earth tides -0.2 .

- Transport Storage Porosity in Consolidated or Unconsolidated Media:

- Resistivity porosity minus effective flow porosity determined by another method -0.5 (but should not be higher than the smaller of the multipliers of the two component estimates).

- Estimates obtained from long-term single-well or cross-hole tracer tests or from measuring the distribution of environmental tracers in flow systems -1.0 .

- Disconnected Storage Porosity in Consolidated or Unconsolidated Media:

- Total porosity estimate minus resistivity porosity -0.5 (but should not be higher than the smaller of the multipliers of the two component estimates).

- Total porosity estimate minus transport storage porosity estimate obtained from long-term single-well or cross-hole tracer tests or from measuring the distribution of environmental tracers in flow systems, minus effective flow porosity estimate obtained by another method 1.0 (but should not be higher than the smaller of the multipliers of any of the three component estimates).

\section{Data Reduction and Analysis Methods}

Data reduction and analysis methods are evaluated independently of the type of porosity measurement being evaluated. However, the analyst must be familiar with state-of-the-art methods in order to make an evaluation. The following multipliers apply (a sliding scale can be employed to allow for a continuum of scores):

- Current and widely-accepted data reduction and analysis method with reputable and traceable calibration information - 1.0. 
- Good method, but calibration information is suspect -0.5 .

- Based on older or less widely-accepted methods, but calibration information is good -0.5 .

- Both methods and calibration information are suspect -0.2 .

Note: Score for any poorly-analyzed dataset can be raised if the raw data are available and are re-analyzed using state-of-the-art methods. However, it is not possible to raise a score above 0.5 for datasets with poor calibration information (unless other information can be used to effectively "recalibrate" the measurements).

\section{Quality of Documentation}

Documentation quality is also evaluated independently of the type of porosity measurement being evaluated. The following multipliers apply (a sliding scale can be employed to allow for a continuum of scores):

- Good (thorough, easy to follow, traceable) - 1.0.

- Appearing in a reputable journal or peer-reviewed report, but otherwise lacking in thoroughness -0.6 .

- $\quad$ Poor -0.3 .

Note: That it is often the case that poor documentation results in the inability to evaluate the data reduction and analysis method (including the calibration information) for a given dataset. Thus, poorly-documented estimates can, in effect, be doubly penalized as a result of both poor documentation scores and poor data reduction and analysis scores.

\subsection{Overall Scores}

The overall score for a given dataset is taken to be the sum of the weights multiplied by the product of the multipliers. For example, if a dataset is assigned component weight scores of 0.5 and 0.2 (for categories 2 and 3, respectively, in Section 4.1), the total weight score is 0.7 . If this dataset is then assigned multipliers of $0.8,1.0$, and 0.6 (for the three respective categories in Section 4.2), the total multiplier score is $0.8 \times 1.0 \times 0.6=0.48$. The overall score is then the product of the weight and multiplier scores, or $0.7 \times 0.48=0.336$. 


\subsection{Documentation Requirements}

For each dataset that is considered for use in CAU-scale flow and transport modeling, the following information should be documented:

- For rejected data, rationale for rejection (i.e., rationale for assigning a total weight score of zero).

- For accepted data, a summary of the data.

- The scores for each weight and multiplier category listed in Section 4.1 and Section 4.2.

- The rationales for the scores for each weight and multiplier category listed in Section 4.1 and Section 4.2.

- The overall weight and multiplier score for the dataset.

A format for documenting this information is not specified, but each of the above elements should be addressed.

Documentation is very important because it is here that the weight and multiplier scores are justified. Assigning the weights and multipliers using the guidance and relative scales provided in Section 4.0 forces the analyst to consider the applicability and relative merits of each dataset. It is not so important that the analyst follow this guidance to the letter, but rather it is important that the analyst documents and justifies the assigned weights and multiplier scores.

Documentation ensures that the analyst's decisions are traceable and easy to review. It also ensures that if additional data are later considered for transfer, these data are scored in a manner consistent with the scoring of previously transferred data. 


\subsection{Use of Weights and Multipliers in Downstream Analyses}

The data that are transferred to a given CAU (as well as CAU-specific data) can be used in many different ways in CAU-scale modeling. It is not the purpose of this document to recommend or exhaustively consider all the possible uses of data or the ways in which the combined weight and multiplier scores should be incorporated into these uses. Rather, a specific and relatively common usage of data is considered, and the manner in which combined weight and multiplier scores are incorporated into other uses can be inferred from this discussion.

It is a common practice to compile cumulative distribution functions (CDFs) of parameter values that are randomly sampled in Monte-Carlo fashion in large-scale models to simulate overall system performance. These CDFs are intended to capture the variability and uncertainty associated with parameter values within the model domain. The large-scale models are typically executed many times so that representative sampling of the component CDFs is accomplished. Finally, a CDF of the model output/predictions is constructed from the multiple model runs to allow an assessment of uncertainty in overall system performance.

Porosity (of any type) is a good example of a parameter for which a CDF may be constructed and then randomly sampled. The suggested method of incorporating the combined weight and multiplier scores into a CDF is best illustrated by example. We assume that there are 5 datasets from which a total of 18 values of matrix porosity have been obtained to represent consolidated rock within a given CAU (this may be from only a certain HSU within the CAU, rather than a bulk value representing the entire CAU). The five datasets are described as follows (the values associated with each separate measurement are listed in parentheses):

- A laboratory dataset with five separate measurements of matrix porosity on different samples taken directly from the $\mathrm{CAU}(0.13,0.16,0.20,0.22$, and 0.23 ).

- A borehole gravimetry log with three measurements of total porosity (assumed to be equivalent to matrix porosity for consolidated rock) taken outside the CAU but in what is considered a similar rock type $(0.12,0.18$, $0.19)$.

- Two neutron logs containing one measurement each from within the CAU $(0.17,0.2)$.

- Three neutron logs containing one measurement each from outside the CAU but in what is considered a similar rock type $(0.12,0.13,0.19)$ 
- Five gamma-gamma logs containing one measurement each from outside the CAU but in what is considered a similar rock type $(0.15,0.18,0.22$, $0.23,0.25)$

Without going into the details of how and why, the combined weight and multiplier scores in Table 6-1 are assigned to the porosity data. Note that the combined scores for the CAU-specific laboratory measurements are all assigned the same value because these measurements are assumed to be conducted as part of a single set of measurements (for this example) using the same analysis method. They are also assumed to be documented in a single report. Using the same combined score for multiple measurements generated within a single study is an acceptable practice when CAU-specific measurements are made in the study.

Table 6-1

\section{Combined Weight and Multiplier Scores \\ for Hypothetical Matrix Porosity Data}

\begin{tabular}{|c|c|c|}
\hline & & \\
\hline & Porosity Value & Combined Score \\
\hline & 0.13 & 0.5 \\
\hline & 0.16 & 0.5 \\
\hline CAU Lab Measurements & 0.20 & 0.5 \\
\hline & 0.22 & 0.5 \\
\hline & 0.23 & 0.5 \\
\hline & 0.12 & 1.2 \\
\hline Non-CAU Gravimetry & 0.18 & 0.7 \\
\hline & 0.19 & 1.0 \\
\hline CAHNoutron I ore & 0.17 & 0.7 \\
\hline CAU INeUtron Logs & 0.2 & 1.0 \\
\hline & 0.12 & 0.6 \\
\hline Non-CAU Neutron Logs & 0.13 & 0.5 \\
\hline & 0.19 & 0.2 \\
\hline & 0.15 & 0.5 \\
\hline & 0.18 & 0.4 \\
\hline Non-CAU Gamma-Gamma & 0.22 & 0.3 \\
\hline & 0.23 & 0.3 \\
\hline & 0.25 & 0.6 \\
\hline
\end{tabular}

When multiple non-CAU measurements are made within a given study, the weight values may vary for the different measurements because the geologic setting of the measurements may have different relevance to the CAU of interest, but the multipliers should be the same (because the same methods and documentation presumably apply to all measurements). Similarly, when measurements on CAU-specific materials are obtained in different investigations and at different times, the weight scores should be the same, but different multipliers can apply because of differences in analytical methods or documentation. Finally, when measurements are obtained on non-CAU materials in different studies and at different times, both the weight scores and multipliers can be different. 
In Table 6-1, the two neutron logs conducted in CAU boreholes were apparently obtained using different analysis methods or had different documentation quality because the scores are different. In the case of the non-CAU measurements (borehole gravimetry, neutron logging, and gamma-gamma logging), the scores are different either because of differences in the relevance of the geologic setting, differences in the analysis methods, differences in documentation quality, or any combination of these differences.

To construct a CDF from the data and combined scores of Table 6-1, it is necessary to sort the porosity values in ascending order and then add the scores in cumulative fashion for each successive porosity value. The cumulative score for each porosity value is then divided by the sum of the scores for all porosity values to obtain an estimate of the cumulative probability for that porosity measurement. Thus, the probability of each porosity value is proportional to its combined score. The results of manipulating the data from Table 6-1 in the manner indicated above are shown in Table 6-2. Figure 6-1 shows the resulting CDF of matrix porosity values.

For this example, the CDF does not appear to be heavily influenced by any single measurement or set of measurements. However, in some cases, a single "very good" measurement may have a combined score equal to or greater than the sum of scores of all the other available data. In such cases, there will be a large jump in the CDF at the value corresponding to the "good" measurement.

Table 6-2

Results of Manipulation of Data in Table 6-1 to Obtain Cumulative Distribution Function of Matrix Porosities

\begin{tabular}{|c|c|c|c|}
\hline Sorted Porosity Value & Combined Score & Cumulative Score & Cumulative Probability \\
\hline \hline 0.12 & 1.2 & 1.2 & 0.114 \\
\hline 0.12 & 0.6 & 1.8 & 0.171 \\
\hline 0.13 & 0.5 & 2.3 & 0.219 \\
\hline 0.13 & 0.5 & 2.8 & 0.267 \\
\hline 0.15 & 0.5 & 3.3 & 0.314 \\
\hline 0.16 & 0.5 & 3.8 & 0.362 \\
\hline 0.17 & 0.7 & 4.5 & 0.429 \\
\hline 0.18 & 0.7 & 5.2 & 0.495 \\
\hline 0.18 & 0.4 & 5.6 & 0.533 \\
\hline 0.19 & 1 & 6.6 & 0.629 \\
\hline 0.19 & 0.2 & 6.8 & 0.648 \\
\hline 0.2 & 0.5 & 7.3 & 0.695 \\
\hline 0.2 & 1 & 8.3 & 0.790 \\
\hline 0.22 & 0.5 & 8.8 & 0.838 \\
\hline 0.22 & 0.3 & 9.1 & 0.867 \\
\hline 0.23 & 0.5 & 9.6 & 0.914 \\
\hline 0.23 & 0.3 & 9.9 & 0.943 \\
\hline 0.25 & 0.6 & 10.5 & 1 \\
\hline
\end{tabular}




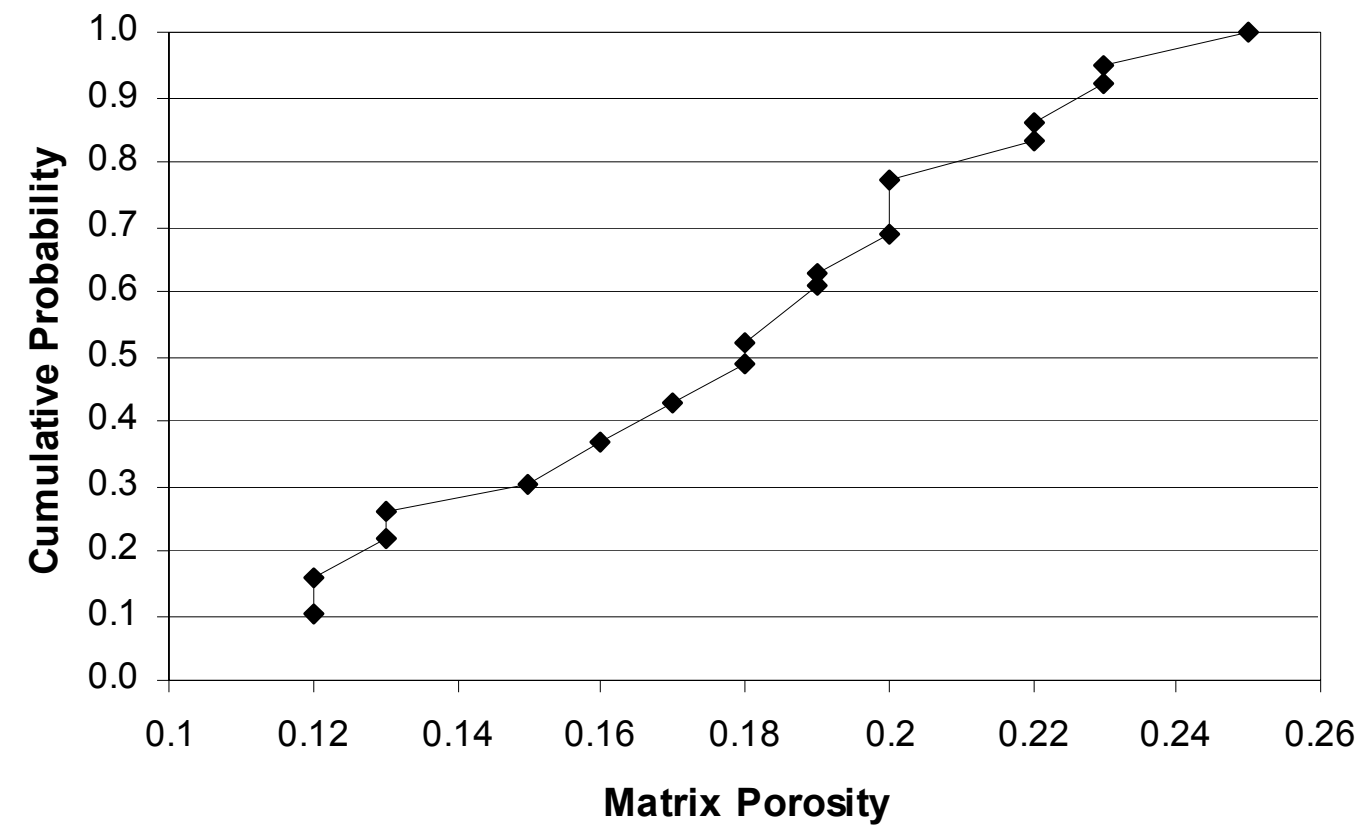

Figure 6-1

Cumulative Distribution Function Associated With the Porosity Data of Table 6-1

This example also serves to illustrate the value of determining combined weight and multiplier scores for CAU-specific data in addition to non-CAU data. Even though the scores for CAU-specific data are not required to determine data transferability, they are essential for establishing a representative CDF.

If another method of data usage is employed in CAU-scale modeling, then the combined weight and multiplier scores should be used in some other manner to "weigh" the available datasets appropriately in the final analysis. The method(s) of translating combined scores into some measure of variability and/or uncertainty should be documented appropriately.

Finally, there are many considerations of variability and uncertainty in parameter values that are not effectively captured by the weight and multiplier system and that go beyond the scope of this attachment. For instance, because weights and multipliers are assigned using relative scales, a CDF generated with only a few data points, data of questionable relevance, and poor-quality measurement methods might look exactly the same as one with many data points, CAU-specific data, and high-quality measurements. However, even though these CDFs may look essentially the same, the uncertainty should be far greater and the confidence far less in the former distribution than the latter one. In general, uncertainty in distributions of parameter values should be considered greater for (1) less total data points, (2) less CAU-specific data, and (3) smaller values of the sum of combined scores divided by the total number of data points. Methods of incorporating these measures of distribution uncertainty into a CAU-scale analysis go beyond the scope of this attachment. 


\subsection{References}

Durham W.B., and B.P. Bonner. 1994. "Self-Propping and Fluid-Flow in Slightly Offset Joints at High Effective Pressures." In J. Geophys. Res. - Solid Earth, Vol. 99, No. B5, pp. 9391-9399.

Freeze, R.A., and J.A. Cherry. 1979. In Groundwater, Prentice Hall, Englewood Cliffs, NJ.

Herring, A.T. 1990. Introduction to Borehole Gravity, EDCON, Inc. Available at website, www.edcon.com.

Keys, W.S. 1997. A Practical Guide to Borehole Geophysics in Environmental Investigations. Boca Raton, FL: CRC Lewis Publishers.

Lee, C.K., Shiang, A.S.T, and Tsay, C.S. 1996. "The Characterization of Porous Solids from Gas Adsorption Measurements." In Porous Ceramic Materials, Vol. 115, pp. 21-43.

Leon y Leon, C.A. 1998. "New Perspectives in Mercury Porosimetry." In $A d v$. Colloids Interface Sci., Vol. 76-77, pp. 341-372.

Liu, L., J.W. Lane, and Y.L. Quan. 1998. "Radar Attenuation Tomography using the Centroid Frequency Downshift Method." In J. Applied Geophys., Vol. 40, No. 1-3, pp. 105-116.

Morland, L.W., and E.C. Donaldson. 1984. "Correlation of Porosity and Permeability of Reservoirs with Well Oscillations Induced by Earth Tides." In Geophys. J. Royal Astronomical Soc., Vol. 79, No. 3, pp. 705-725.

Reimus, P.W. 2003. "A Refined Approach to Estimating Effective Flow Porosity from Cross-Hole Tracer Tests in Fractured Media." In Proceedings of the $10^{\text {th }}$ International High-Level Radioactive Waste Management Conference, American Nuclear Society.

Todd, D.K. 1959. In Ground Water Hydrology. New York, NY: John Wiley and Sons.

Wilson, J.E., Goodwin, L.B., and Lewis, C.S. "Deformation Bands in Non-Welded Ignimbrites: Petrophysical Controls on Fault-Zone Deformation and Evidence for Preferential Fluid Flow. Submitted to Geology. 
Attachment B

Property Description - Matrix Diffusion 


\section{Table of Contents}

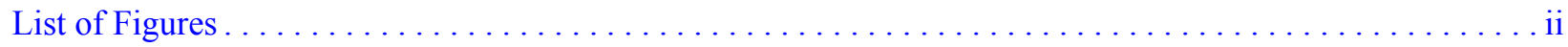

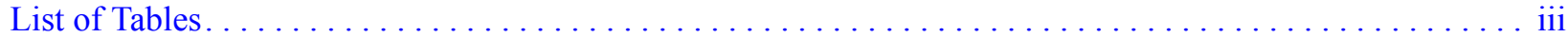

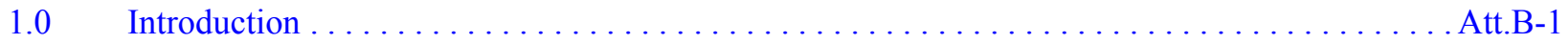

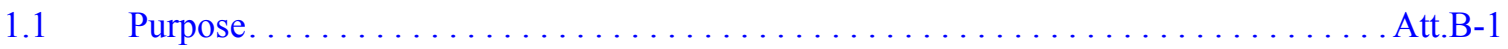

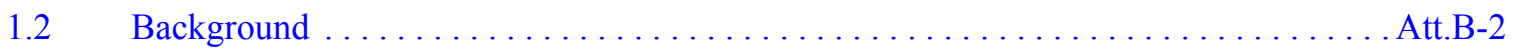

1.3 Summary of Parameters Affecting Matrix Diffusion at Field Scales . . . . . . . . . . Att.B-7

1.4 Scope of Attachment . . . . . . . . . . . . . . . . . . . . . . . Att.B-10

$2.0 \quad$ Measurements of Matrix Diffusion Parameters. . . . . . . . . . . . . . . . . . . . . . Att.B-12

$2.1 \quad$ Laboratory Measurements of Matrix Diffusion Coefficients . . . . . . . . . . . . . Att.B-12

2.2 Field Measurements of Matrix Diffusion Parameters . . . . . . . . . . . . . Att.B-18

2.2.1 Matrix Diffusion Mass Transfer Coefficients ................... Att.B-18

2.2.2 Flowing Fracture Spacing and Fracture Apertures $\ldots \ldots \ldots \ldots \ldots \ldots$ Att.B-20

2.2.3 Insights from Geochemical and Isotopic Analyses of Groundwaters . . . . . Att.B-23

2.3 Summary of Measurement Methods . . . . . . . . . . . . . . . . . . . Att.B-24

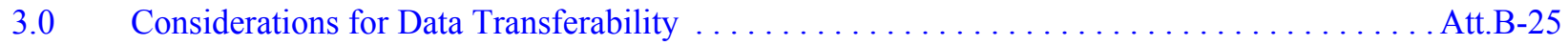

3.1 Importance of Data in CAU-Scale Transport Modeling $\ldots \ldots \ldots \ldots \ldots \ldots \ldots$ Att.B-25

3.2 Dependence of Matrix Diffusion Coefficients on Matrix Porosity and Permeability . . . . . . . . . . . . . . . . . . . . . . . . . . . Att.B-26

3.3 Dependence of Matrix Diffusion Coefficients on the Geologic Setting . . . . . . . . . . Att.B-27

3.4 Dependence of Other Matrix Diffusion Parameters on the Geologic Setting . . . . . . . Att.B-27

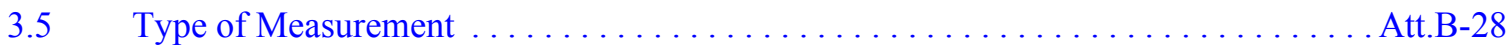

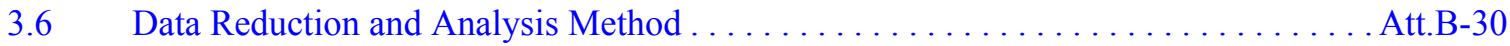

3.7 Quality of Documentation . . . . . . . . . . . . . . . . . . . . . Att.B

3.8 Dependence of Matrix Diffusion Coefficients on Solute Characteristics,

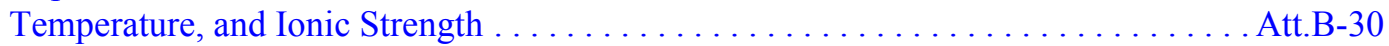

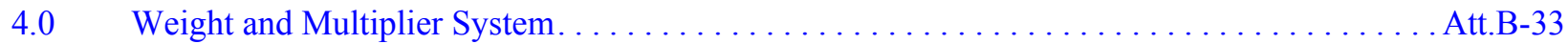

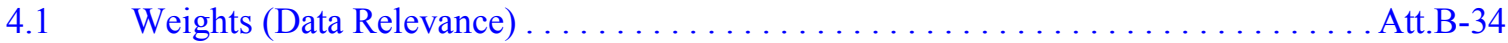

4.2 Multipliers (Measurement Method, Quality of Analyses and Documentation). . . . . Att.B-36

$4.3 \quad$ Overall Scores . . . . . . . . . . . . . . . . . . . . . . . . . . . . . . . Att.B-38

$5.0 \quad$ Documentation Requirements . . . . . . . . . . . . . . . . . . . . . . . . . . . . . . . . Att.B-39

$6.0 \quad$ Use of Weights and Multipliers in Downstream Analyses . . . . . . . . . . . . . . . . . . . . . Att.B-40

$7.0 \quad$ References . . . . . . . . . . . . . . . . . . . . . . . . . . . . . . . . . . . . Att.B-45 


\section{List of Figures}

Number

Title

Page

1-1 Simplified geometry of a dual-porosity system used in this attachment.

The matrix porosity is $\phi$, and the porosity within the fractures is $\eta$. Fracture

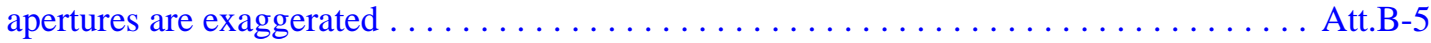

1-2 Solute responses to a step-function input in a dual-porosity system with the geometry on Figure1-1 as a function of $\mathrm{R}_{\mathrm{f}}\left(\mathrm{GWTT}_{\mathrm{f}}\right) \mathrm{T}_{\mathrm{D} \text {, char }}$ (numbers next to curves). The bold curve is the response with no matrix diffusion. $\mathrm{R}_{\mathrm{md}} \approx 10$ for all curves except the bold curve. The mean arrival time for all cases with matrix diffusion is the same (250 time units). Curves generated using the RELAP computer model . . . . Att.B-6

1-3 Solute responses in a dual-porosity system as a function of Rf (GWTTf) $\mathrm{T}_{\mathrm{D} \text {,char }}$ (numbers next to curves) for a system with twice the average fracture aperture of Figure1-2 while holding all other parameters constant. The bold curve is the response with no matrix diffusion. $\mathrm{R}_{\mathrm{md}} \approx 5$ for all curves except the bold curve. Curves generated

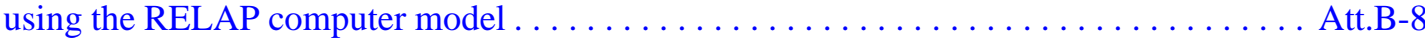

2-1 Schematic Illustration of a Diffusion Cell Experiment $\ldots \ldots \ldots \ldots \ldots \ldots \ldots \ldots$. Att.B-13

2-2 Hypothetical cross-hole tracer trest responses of nonsorbing solutes with different diffusion coefficients in a single-porosity system and a dual-porosity system. Curves generated using the RELAP computer model . . . . . . . . . . . . . Att.B-19

2-3 Differences in multiple nonsorbing tracer responses in cross-hole tracer test with an "infinite" matrix (no diffusion boundary) and a finite matrix (diffusion boundary).

Note the greater difference in the tail concentrations of the two tracers for the finite matrix. Curves generated using the RELAP computer model $\ldots \ldots \ldots \ldots \ldots \ldots \ldots \ldots$ Att.B-22

6-1 Cumulative Distribution Function Associated With the Matrix Diffusion Coefficient Data of Table6-1 


\section{List of Tables}

Number

Title

Page

3-1 Factor by Which Diffusion Coefficients Change as a Function of

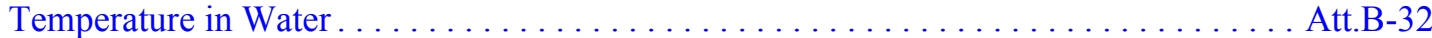

6-1 Combined Weight and Multiplier Scores for Hypothetical Matrix Diffusion

Coefficient Data . . . . . . . . . . . . . . . . . . . . . . . . . . . . . . . . . . . Att.B-42

6-2 Results of Manipulation of Data in Table 6-1 to Obtain Cumulative

Distribution Function of Matrix Diffusion Coefficients. . . . . . . . . . . . . . . . . Att.B-43 


\subsection{Introduction}

\subsection{Purpose}

The purpose of this attachment is to provide guidance on transferring matrix diffusion data obtained from outside CAUs of interest at the NTS to the UGTA Project so that the data can be used to parameterize matrix diffusion in flow and radionuclide transport models. These models will be used to establish CAU boundaries, which will roughly correspond to perimeters within which future access to groundwater will be controlled owing to potential radioactive contamination originating from nuclear test cavities. Because radionuclide transport modeling for the UGTA project is focused exclusively on transport below the water table, only matrix diffusion under saturated conditions is considered in this attachment. Also, radionuclide decay is not considered in this attachment because there is no coupling between the effects of decay on transport and the effects of matrix diffusion.

This attachment addresses the following data transferability factors and considerations mentioned in the main body of this document:

1. Parameter characteristics, including underlying dependencies on material properties or other parameters,

2. Similarity of donor and receptor HSUs, with respect to geologic setting and other relevant characteristics for the parameter of concern,

3. Type of measurement and/or interpretative technique, including measurement scale,

4. Quality of documentation of measurements and interpretive methods, and

5. Considerations in assigning relative weights to different parameter datasets.

This attachment does not directly address the following data transferability factors and considerations that are discussed in the main body of this document.

Although all of these factors are discussed briefly as they relate to the above-listed factors:

1. Modeling approaches, including conceptual models and model scale,

2. Heterogeneity,

3. Ranges in values, and 
4. Sensitivity of contaminant boundaries to parameter values.

Section 1.2 provides a background discussion on matrix diffusion, including the effect that matrix diffusion has on contaminant transport. It is pointed out in Section 1.3 that the scope of this attachment is limited to the transferability of matrix diffusion coefficient data, and it is intended primarily to address matrix diffusion in fractured geologic media. However, the discussions throughout the attachment can also be applied to interlayered/juxtaposed unfractured media (including sediments and alluvium) in which there is a significant volume fraction of stagnant or near-stagnant water. The attachment is not intended to address the transfer of geometric parameters that affect matrix diffusion, nor does it address matrix porosity, which, although very important for matrix diffusion, is covered in Attachment A of this document. Section 2.0 presents different methods of measuring matrix diffusion parameters in both the lab and the field. In Section 3.0, various considerations involved in the transfer of matrix diffusion data are discussed. Section 4.0 then presents a recommended methodology for transferring matrix diffusion data, and Section 5.0 provides an example of applying this methodology.

\section{$1.2 \quad$ Background}

Matrix diffusion is the diffusion of solutes from flowing water (generally in fractures) into stagnant or nearly-stagnant water contained in the pores of consolidated rock matrices (Neretnieks, 1980). The process is reversible, so solutes that diffuse into the matrix can also diffuse back out. Because consolidated rock matrices typically have permeabilities that are orders of magnitude lower than open fractures (under saturated conditions), the vast majority of the volumetric flow in such systems (called "dual-porosity" systems) usually occurs in fractures, even though the majority of the total system porosity (and hence volume of water) may be contained in the matrix pores. Solute transport rates under these conditions can be significantly attenuated relative to water flow rates in fractures because solutes can diffuse into the matrix and spend a significant fraction of their total residence time in the system in stagnant water.

It is widely recognized that diffusion coefficients in porous media are smaller than free-water diffusion coefficients for any given solute. The reduction in diffusion coefficient in porous media relative to free water is a consequence of the tortuous nature of the pathways that solutes must follow when they diffuse through the media. The relationship between diffusion coefficients in porous media and free water is frequently expressed as (Neretnieks, 1980):

$$
D_{\mathrm{m}}=\left(\frac{\kappa}{\tau^{2}}\right) D_{\mathrm{s}}
$$

where

$$
\begin{aligned}
D_{\mathrm{m}}= & \text { solute diffusion coefficient in porous medium (i.e., matrix diffusion } \\
& \text { coefficient), } \mathrm{cm}^{2} / \mathrm{sec} \\
D_{\mathrm{s}}= & \text { solute diffusion coefficient in free water, } \mathrm{cm}^{2} / \mathrm{sec} \\
\kappa & =\text { constrictivity factor, and } \\
\tau \quad= & \text { tortuosity factor }
\end{aligned}
$$


This relationship is often more succinctly expressed as (e.g., Freeze and Cherry, 1979):

$$
D_{\mathrm{m}}=\omega D_{\mathrm{s}}
$$

where $\omega=$ is an empirical coefficient often called the tortuosity (which has a different meaning than the tortuosity factor in equation B-1). $\omega$ typically takes on values between 0.01 and 0.5 , although it can be smaller in very tortuous media. Note that there is not a direct dependence of the matrix diffusion coefficient on porosity, but $\omega$ tends to be positively correlated with porosity, as will be discussed later in this attachment.

Following the reasoning originally presented by Maloszewski and Zuber (1991), an effective solute retardation factor in a system caused by matrix diffusion (with no adsorption in either the matrix or fractures), $\mathrm{R}_{\mathrm{MD}}$, can be defined as

$$
\mathrm{R}_{\mathrm{MD}}=1+\frac{\text { time spent in stagnant water }}{\text { time spent in flowing water }}
$$

For a solute that experiences adsorption, equation (B-3) becomes

$$
\mathrm{R}_{\mathrm{MD}}=\mathrm{R}_{\mathrm{f}}+\frac{\text { (time spent in stagnant water) } \mathrm{R}_{\mathrm{m}}}{\text { time spent in flowing water }}
$$

where

$\mathrm{R}_{\mathrm{m}}=1+\frac{\rho_{\mathrm{B}} \mathrm{K}_{\mathrm{d}}}{\phi}=$ retardation factor in matrix caused by sorption processes

$\mathrm{R}_{\mathrm{f}}=1+\frac{\rho_{\mathrm{f}} \mathrm{K}_{\mathrm{d}}}{\eta}=$ retardation factor in fractures

$\mathrm{K}_{\mathrm{d}}=$ the solute partition coefficient in the matrix $(\mathrm{ml} / \mathrm{g})$,

$\mathrm{K}_{\mathrm{df}}=$ the solute partition coefficient in fractures $(\mathrm{ml} / \mathrm{g})$,

$\phi=$ the matrix porosity,

$\rho_{\mathrm{B}}=$ the matrix bulk density $\left(\mathrm{g} / \mathrm{cm}^{3}\right)$,

$\rho_{\mathrm{f}} \quad=$ the effective bulk density in fractures $\left(\mathrm{g} / \mathrm{cm}^{3}\right)$, and

$\eta \quad=$ the porosity within fractures.

Note that if fractures that do not contain granular fill material, they will have $\eta=1$, but $\rho_{\mathrm{f}}$ will not necessarily have a value of zero because solutes can still adsorb to fracture walls. Sorption to fracture walls may be especially strong when the walls have secondary mineral coatings with a high sorption capacity and/or with redox-active surfaces (e.g., iron or manganese oxides). In this case, it may be more appropriate to define the fracture retardation factor as $1+\frac{\mathrm{K}_{\mathrm{Af}}}{b}$, 
where

$\mathrm{K}_{\mathrm{Af}}=$ surface-area-based partition coefficient in fractures $\left(\mathrm{cm}^{3} / \mathrm{cm}^{2}\right)$, and

$b=$ the half the fracture aperture $(\mathrm{cm})$, which is also the volume to surface area ratio in a parallel-plate fracture $\left(\mathrm{cm}^{3} / \mathrm{cm}^{2}\right)$.

The fracture retardation factor can become quite complicated if fracture coatings have internal porosity and varying mineralogy. However, for the purposes of this attachment, these additional complications are not addressed further; it is sufficient to simply recognize that many factors may contribute to $\mathrm{R}_{\mathrm{f}}$.

Equation (B-4) is a more general definition of $\mathrm{R}_{M D}$ than equation (B-3), as $\mathrm{R}_{\mathrm{m}}$ and $\mathrm{R}_{\mathrm{f}}$ can be set equal to 1 for nonsorbing solutes. When sorbing solutes are considered, $\mathrm{R}_{\mathrm{MD}}$ should properly be referred to as the retardation factor accounting for matrix diffusion rather than caused by matrix diffusion, because retardation in fractures (or flowing porosity in general) will occur even without matrix diffusion. Also, a portion of the retardation in the matrix can be attributed to sorption in the matrix if $R_{m}$ is greater than 1 .

Figure 1-1 depicts a simplified geometry of a dual-porosity system that will be used for convenience in further discussion. $L$ is the characteristic distance between flowing fractures in the system $(\mathrm{cm})$, and $b$ is the characteristic half-aperture of the flowing fractures $(\mathrm{cm})$. The matrix porosity is $\phi$, and the porosity within the fractures is $\eta$. L/2 could also be considered the characteristic distance from the centerline of a flowing fracture to a diffusion boundary within the matrix (where a no-flux boundary condition is imposed). This latter interpretation allows for the possibility that the matrix may not be completely accessible to solutes by diffusion.

In the conceptual model of Figure 1-1, flow is assumed to occur only in the $\mathrm{x}$-direction in fractures. The matrix is assumed to contain stagnant water that has zero velocity, and solute diffusion occurs between the fractures and matrix in a direction perpendicular to the fractures. Grisak and Pickens (1980) showed that these simplifying assumptions provide reasonable approximations of the effects of matrix diffusion in systems in which flow velocities in the matrix are as much as about 1 percent of the flow velocity in fractures. Based on available information (Rehfeldt et al., 2004), most fractured-rock aquifers at the NTS should have fracture-matrix permeability contrasts of greater than two orders of magnitude, so the work of Grisak and Pickens (1980) suggests that the conceptual model and simplifying assumptions should be a valid approximation for fractured rock aquifers at the NTS.

Using the simplified geometry of Figure 1-1, the ratio of stagnant to flowing water volume in the system is given by

$$
\frac{\text { stagnant water volume }}{\text { flowing water volume }}=\frac{\phi(L / 2-b \eta)}{b \eta}
$$



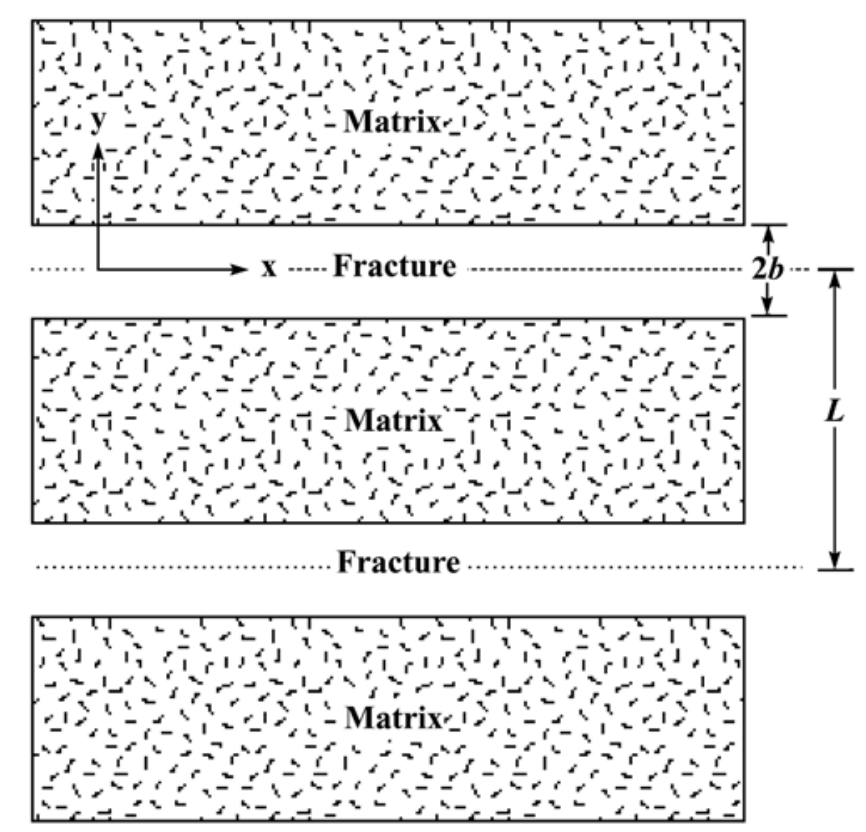

Figure 1-1

Simplified geometry of a dual-porosity system used in this attachment. The matrix porosity is $\phi$, and the porosity within the fractures is $\eta$. Fracture apertures are exaggerated

This quantity is also equal to the ratio of time spent in stagnant water vs. flowing water in the system for a nonsorbing solute, and hence it determines the effective retardation factor caused by matrix diffusion without the effects of sorption:

$$
\mathrm{R}_{\mathrm{MD}}=1+\frac{\phi(L / 2-b \eta)}{b \eta} \text { for a nonsorbing solute }
$$

Figure 1-2 shows the effect that matrix diffusion has on solute transport (step-function input) in a dual-porosity system with the geometry of Figure 1-1. The $\mathrm{x}$ axis in this figure is an arbitrary time scale. The bold curve shows the solute response in the system assuming no matrix diffusion. This response is governed by the groundwater travel time through fractures (or flowing porosity in general) in the system, $G W T T_{f}$, multiplied by $\mathrm{R}_{\mathrm{f}} . G W T T_{f}$ is defined as

$$
G W T T_{f}=\frac{\mathrm{X}}{\mathrm{Q}}\left(\frac{L}{2 b \eta}\right)
$$




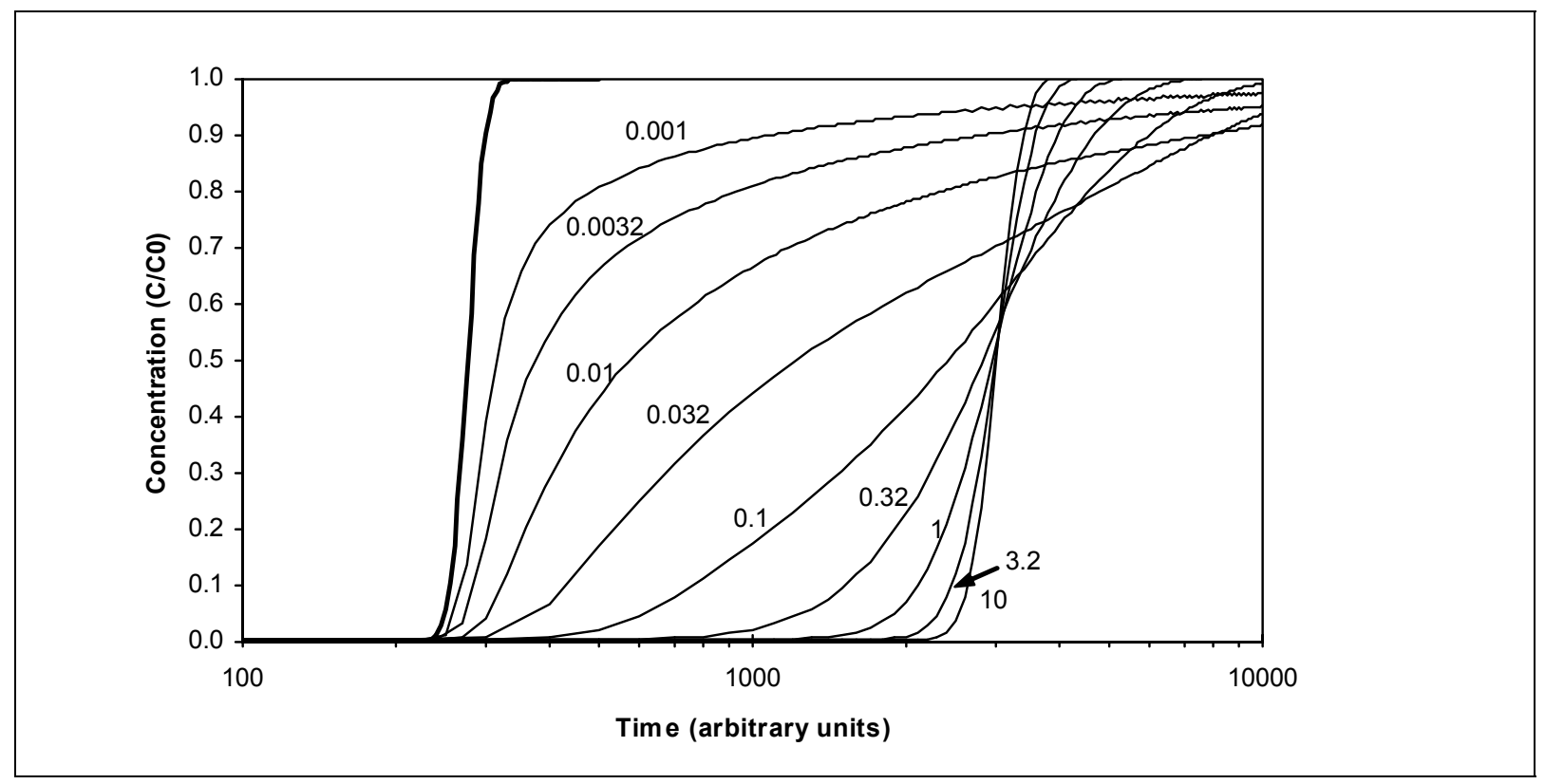

Figure 1-2

Solute responses to a step-function input in a dual-porosity system with the geometry on Figure 1-1 as a function of $R_{f}\left(G W T T_{f}\right) T_{D, c h a r}$ (numbers next to curves). The bold curve is the response with no matrix diffusion. $\mathbf{R}_{\mathrm{md}} \approx 10$ for all curves except the bold curve. The mean arrival time for all cases with matrix diffusion is the same (250 time units). Curves generated using the RELAP computer model (Reimus and Haga, 1999)

where

$\mathrm{X}=$ total travel distance $(\mathrm{cm})$ and

$\mathrm{Q}=$ groundwater specific discharge (volumetric flow rate per unit cross section of the system, $\mathrm{cm}^{3} / \mathrm{cm}^{2}$-sec), and

$2 b \eta / L=$ overall flow porosity in the system.

Different solute breakthrough curves are shown in Figure 1-2 for different ratios of the solute travel time through fractures, $\mathrm{R}_{\mathrm{f}}\left(G W T T_{\mathrm{f}}\right)$, to the characteristic diffusion time in the system, $T_{D, \text { char }}$ given by

$$
T_{D, \text { char }}=\frac{\mathrm{R}_{m} L^{2}}{4 D_{m}}
$$

$D_{\mathrm{m}}=$ the solute matrix diffusion coefficient $\left(\mathrm{cm}^{2} / \mathrm{sec}\right)$.

$T_{D, \text { char }}=$ can be considered a measure of the time it takes a solute to diffuse halfway through the matrix to neighboring flowing fractures (or to a no-flux boundary).

Figure 1-2 shows that when $\mathrm{R}_{\mathrm{f}}\left(G W T T_{f}\right)$ is significantly less than $T_{D, \text { char }}$, a portion of the solute mass arrives at nearly the groundwater travel time in fractures. However, an extremely long tail is observed because some of the solute mass diffuses into the matrix and remains for a very long time. When fracture travel 
times are significantly longer than the characteristic diffusion time, the entire solute mass arrives within a relatively narrow range of times, $T_{\text {solute }}$, centered around:

$$
T_{\text {solute }}=G W T T_{f}\left[\mathrm{R}_{\mathrm{f}}+\frac{\mathrm{R}_{\mathrm{m}} \phi(L / 2-b \eta)}{b \eta}\right]
$$

Note that the expression in brackets in equation (B-9) is equivalent to $R_{M D}$ in equation (B-6) but with retardation factors for sorption in the fractures and matrix included. It is important to recognize that the mean solute travel time is the same for all of the curves of Figure 1-2 except the first one (with no matrix diffusion). However, the fraction of mass arriving early varies significantly and depends strongly on the ratio of $T_{\text {solute }}$ to $T_{D, \text { char }}$. Thus, the first arrival or "breakthrough" time, which is usually arbitrarily defined as the arrival time of some small percentage of solute mass (e.g., 1 percent), as well as the median travel time (time associated with the arrival of 50 percent of the mass) are strongly dependent on this ratio. If CAU boundaries are determined based on these measures of predicted radionuclide transport, then it will be very important to estimate $T_{\text {solute }} / T_{D, \text { char }}$

Figure 1-3 shows the effect of doubling the flowing porosity (by doubling fracture apertures) relative to Figure 1-2 while holding all other parameters constant. Doubling the fracture apertures reduces the effective retardation factor by approximately a factor of two because it doubles the volume of flowing water in the system without significantly affecting the stagnant water volume in the system. It also decreases the effective diffusive mass transfer rate between fractures and matrix, which results in earlier first arrival times and a greater fraction of solute mass arriving early for the same value of $T_{D, \text { char }}$ as in Figure 1-2.

The diffusive mass transfer rate between fractures and matrix is governed by a lumped parameter called the diffusive mass transfer coefficient, $M T C_{D}$, given by

$$
M T C_{D}=\frac{\phi}{b \eta} \sqrt{D_{\mathrm{m}} \mathrm{R}_{\mathrm{m}}}
$$

This parameter has units of time $\mathrm{i}^{-1 / 2}$, so $M T C_{D}^{2}=\frac{\phi^{2}}{b^{2} \eta^{2}} D_{m} \mathrm{R}_{m}$, which has units of time ${ }^{-1}$, is also frequently used as the effective mass transfer coefficient for matrix diffusion. However, in this attachment, $M T C_{D}$ will be referred to as the mass transfer coefficient. When $M T C_{D}$ is small and solute travel times through fractures are short, a significant fraction of the solute mass will move through the system as if the matrix is not present, regardless of what the overall effective retardation factor is. This fraction will depend primarily on the value of $M T C_{D}$.

\subsection{Summary of Parameters Affecting Matrix Diffusion at Field Scales}

In summary, there are three primary lumped parameters that quantify how matrix diffusion affects solute transport in large-scale contaminant transport models. These parameters are: 


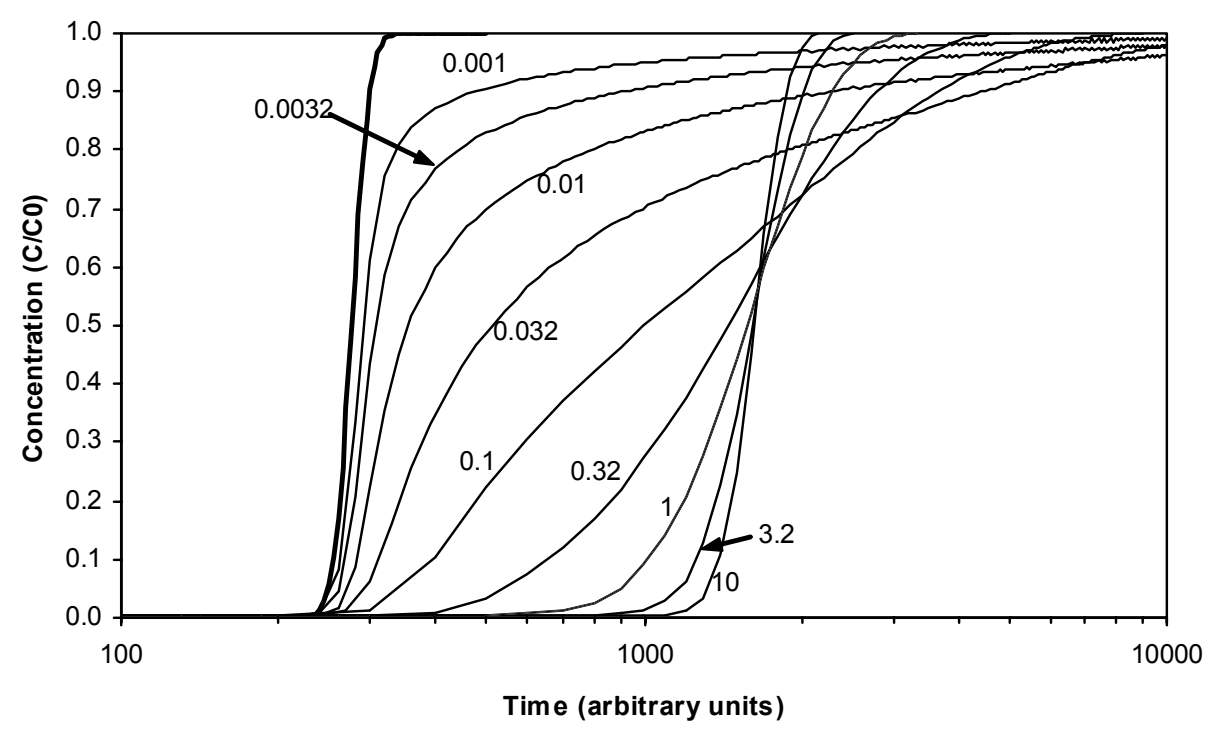

Figure 1-3

Solute responses in a dual-porosity system as a function of $R_{f}\left(G W T T_{f}\right) T_{D, c h a r}$ (numbers next to curves) for a system with twice the average fracture aperture of Figure 1-2 while holding all other parameters constant. The bold curve is the response with no matrix diffusion. $R_{\mathrm{md}} \approx 5$ for all curves except the bold curve. Curves generated using the RELAP computer model (Reimus and Haga, 1999)

1. Overall Solute Retardation Factor accounting for Matrix Diffusion, $\mathrm{R}_{\mathrm{MD}}$ - Using the idealized system geometry described in Section 1.2, this retardation factor is equal to $\mathrm{R}_{\mathrm{f}}+\frac{\mathrm{R}_{\mathrm{m}} \phi(L / 2-b \eta)}{b \eta}$ (term in brackets in equation B-9). Thus, the retardation factor does not depend on the matrix diffusion coefficient $\left(D_{\mathrm{m}}\right)$, but rather it depends on solute sorption characteristics, matrix porosity, fracture spacing, fracture apertures, and internal fracture porosity.

2. Characteristic Time for Matrix Diffusion, $T_{\mathrm{D}, \mathrm{char}}-$ Again using the idealized geometry of Section 1.2, this characteristic time is equal to $\frac{\mathrm{R}_{\mathrm{m}} L^{2}}{4 D_{m}}$. If $\mathrm{R}_{\mathrm{f}}\left(G W T T_{f}\right)$ exceeds this time, then the vast majority of the solute mass will experience the effective retardation factor given above. If not, only a portion of the mass will be significantly retarded. $T_{D, \text { char }}$ depends on $D_{\mathrm{m}}$, fracture spacing, and solute sorption characteristics. 
3. Mass Transfer Coefficient for Matrix Diffusion, $M T C_{D}$ - The matrix diffusion mass transfer coefficient is given by $\frac{\phi}{b \eta} \sqrt{D_{\mathrm{m}} \mathrm{R}_{\mathrm{m}}}$, assuming the geometry of Figure 1-1. This parameter is important primarily for determining the fraction of solute mass that experiences very little retardation when $\mathrm{R}_{\mathrm{f}}\left(G W T T_{f}\right)$ is shorter than the $T_{D, \text { char }}$ If $\mathrm{R}_{\mathrm{f}}\left(G W T T_{f}\right)$ is longer than $T_{D, \text { char }}$, then solute transport will be relatively insensitive to the mass transfer coefficient.

These three lumped parameters are formed from various combinations of five physical parameters, $\phi, b, \eta, L$, and $D_{\mathrm{m}}$, and two chemical parameters, $\mathrm{R}_{\mathrm{f}}$ and $\mathrm{R}_{\mathrm{m}}$. The first four parameters describe the physical nature of the system and are used to quantify the ratio of accessible stagnant water to flowing water volume in the system, and hence the effective retardation factor caused by matrix diffusion alone (without the effects of sorption). $\phi, b, \eta, D_{\mathrm{m}}$, and $\mathrm{R}_{\mathrm{m}}$ combine to describe the effective diffusive mass transfer rate between flowing and nonflowing portions of the system (i.e., fractures and matrix). Although this mass transfer rate does not affect the overall solute retardation factor, it does quantify the fraction of solute mass that moves relatively quickly through the system. Lower mass transfer rates will result in higher fractions of solute mass arriving early, regardless of the value of the effective retardation factor. $T_{D, \text { char }}$ (equation B-8) is somewhat related to the mass transfer rate. When the solute travel time through fractures in the system, $\mathrm{R}_{\mathrm{f}}$ $\left(G W T T_{f}\right)$ exceeds $T_{D, c h a r}$, the mass transfer rate becomes less important, and the vast majority of the solute mass will move through the system with a retardation factor given by $\mathrm{R}_{\mathrm{MD}}$.

The seven parameters used to describe matrix diffusion (above) are, for the most part, independent. However, $\mathrm{R}_{\mathrm{m}}$ and $D_{\mathrm{m}}$ are dependent on $\phi$, with the former being negatively correlated and the latter tending to be positively correlated with $\phi$ (see Section 3.2 and equation B-17). $\mathrm{R}_{\mathrm{f}}$ is also dependent on $\eta$. All seven parameters will likely vary in a given rock mass such that each can be described by statistical distributions having a mean and variance (as well as other parameters necessary to describe the distributions). Variability in matrix diffusion parameters in dual-porosity media can be addressed in models using multi-rate diffusion approaches such as that described by Haggerty and Gorelick (1995).

It should be pointed out that the simplified geometric conceptualization of matrix diffusion shown in Figure 1-1 and the notation used above are not uniform and consistent in the literature. Matrix diffusion can be conceptualized as diffusion into spherical or cylindrical matrix blocks rather than rectangular blocks. In these cases, the expressions for characteristic times, relative volumes, and retardation factors are slightly different than those presented above. However, the general concepts are the same, and the lumped parameters derived for different geometries always have the same effect on matrix diffusion. Furthermore, they can be related to each other by simple geometric factors. Additional complexity can also be built into matrix diffusion conceptualizations. For instance, thin fracture coating minerals that slow diffusion rates into and out of the matrix can be accounted for using a fracture "skin" mass transfer coefficient (e.g., Moench, 1995). 


\subsection{Scope of Attachment}

It is clear from the previous section that the transferability of matrix diffusion data from one location to another should properly consider the transferability of each of the seven parameters that govern matrix diffusion (discussed at the end of Section 1.2 and appearing in Section 1.3). The transferability of matrix porosity data is addressed in Attachment $\mathrm{A}$ of this document, so it will not be discussed here except as it relates to the transferability of matrix diffusion data. The transferability of sorption parameters $\left(\mathrm{K}_{\mathrm{d}}, \mathrm{R}_{\mathrm{f}}\right.$, or $\mathrm{R}_{\mathrm{m}}$ values) are not addressed in this attachment because, although sorption magnifies the effects of matrix diffusion, it is a chemical process that is otherwise independent of the physical process of diffusion. Only physical processes and parameters that affect nonsorbing radionuclides (as well as sorbing radionuclides) are considered in this attachment.

$b, \eta$, and $L$ are geometric parameters that are likely to be location specific because they depend not only on rock mass characteristics, but also on stress and strain history within the rock mass. The transferability of $b$ and $L$ is addressed in this attachment, although the transfer of such data should be recognized as being highly uncertain. Transfer of $\eta$ is not addressed in this attachment because very little data on $\eta$ exist in any media.

This attachment focuses primarily on the transferability of $D_{\mathrm{m}}$ data. $D_{\mathrm{m}}$ appears in two of the three lumped parameters summarized in Section 1.3, and it must be known reasonably well to estimate the ratio $T_{\text {solute }} / T_{D, \text { char }}$, which determines whether a significant fraction of solute mass will travel faster than $\mathrm{R}_{\mathrm{f}}\left(G W T T_{f}\right)$. Although $D_{\mathrm{m}}$ will ultimately be less important in quantifying the overall influence of matrix diffusion on solute transport than $\phi, b$, or $L$ when $T_{\text {solute }} / T_{D, \text { char }}$ is large, an estimate of $D_{\mathrm{m}}$ is still necessary to make this determination.

The above discussion and Section 1.2 focus primarily on matrix diffusion in fractured media. However, matrix diffusion, or, more specifically, diffusion between flowing and stagnant water, can also occur in interlayered/juxtaposed unfractured media (including sediments and alluvium). In such media, stagnant water may be present either in layers of fine-grained sediments such as clay, interstitial pore spaces filled with very fine-grained materials, or within the internal porosity of the grains (or cobbles) of the media. However, the ratio of stagnant to flowing water volume in unconsolidated media is usually much smaller than in fractured media. Therefore, this attachment is intended to apply primarily to fractured media. Also, as mentioned in Section 1.1, the attachment considers only water-saturated conditions, and it does not consider radioactive decay. Clearly, if $T_{\text {solute }}$ is nearly equal to or greater than the half-life of a radionuclide, then analysts should account for radioactive decay in predictive transport calculations.

This attachment does not consider geometric and structural complexities/ heterogeneities that could result in highly irregular boundary conditions for matrix diffusion and/or spatial variability in parameters describing matrix diffusion. Although these complexities certainly exist in nature, the intent of this attachment is to address the basic concepts of matrix diffusion and factors that affect the 
transferability of matrix diffusion data. This intent can best be met by simplifying geometric and structural representations of groundwater flow systems. Such simplification will also ultimately have to be done in CAU-scale transport modeling. 


\subsection{Measurements of Matrix Diffusion Parameters}

Matrix diffusion parameters can be measured in both the laboratory and the field. Lab measurements are best suited for obtaining direct estimates of matrix diffusion coefficients $\left(D_{\mathrm{m}}\right)$ while field measurements are more suited to obtaining estimates of matrix diffusion mass transfer coefficients, $M T C_{D}$. However, certain types of field measurements can also provide crude or bounding estimates of $L / 2$ and $b$. Laboratory measurements are discussed in Section 2.1, and field measurements are discussed in Section 2.2.

\subsection{Laboratory Measurements of Matrix Diffusion Coefficients}

\section{Diffusion Cell Experiments}

Laboratory measurements of matrix diffusion coefficients can be obtained by several different, but related, methods. Shackleford (1991) provides a good overview of these methods. One approach is the "diffusion cell" or "through-diffusion" experiment, in which two reservoirs are separated by a porous medium in which the measurement is made (note that the porous medium is often a block of consolidated rock matrix cut into appropriate dimensions). A diffusion cell experimental apparatus is illustrated schematically in Figure 2-1. Although one of the reservoirs in Figure 2-1 is much larger than the other, this is not a requirement for a diffusion cell experiment. The porous medium separating the two reservoirs is pre-saturated prior to the experiment, and both reservoirs are filled with groundwater. A high concentration of solute is then introduced into one reservoir while the other reservoir is initially solute-free. In Figure 2-1, the large reservoir is the high-concentration reservoir, and the small reservoir is kept well-mixed and continuously flushed so that the solution in this reservoir can be continuously collected for solute analyses. This configuration assures that a reasonably high concentration gradient is maintained across the porous medium throughout the experiment. It also increases the chances of achieving a quasi-steady state in which concentrations throughout the system remain nearly constant (after sufficient time has passed).

The horizontal orientation of the system in Figure 2-1 is intended to minimize head or pressure gradients across the medium to ensure that solute transport is by diffusion only, without advection. Diffusion coefficient estimates are obtained by measuring either (1) the increase in solute concentration in the water flushed from initially solute-free reservoir, (2) the decrease in concentration in the high-concentration reservoir over time, (3) the concentration difference between inlet and outlet reservoirs when a quasi-steady state is reached (i.e., when concentrations no longer change significantly with time), or (4) a combination of 


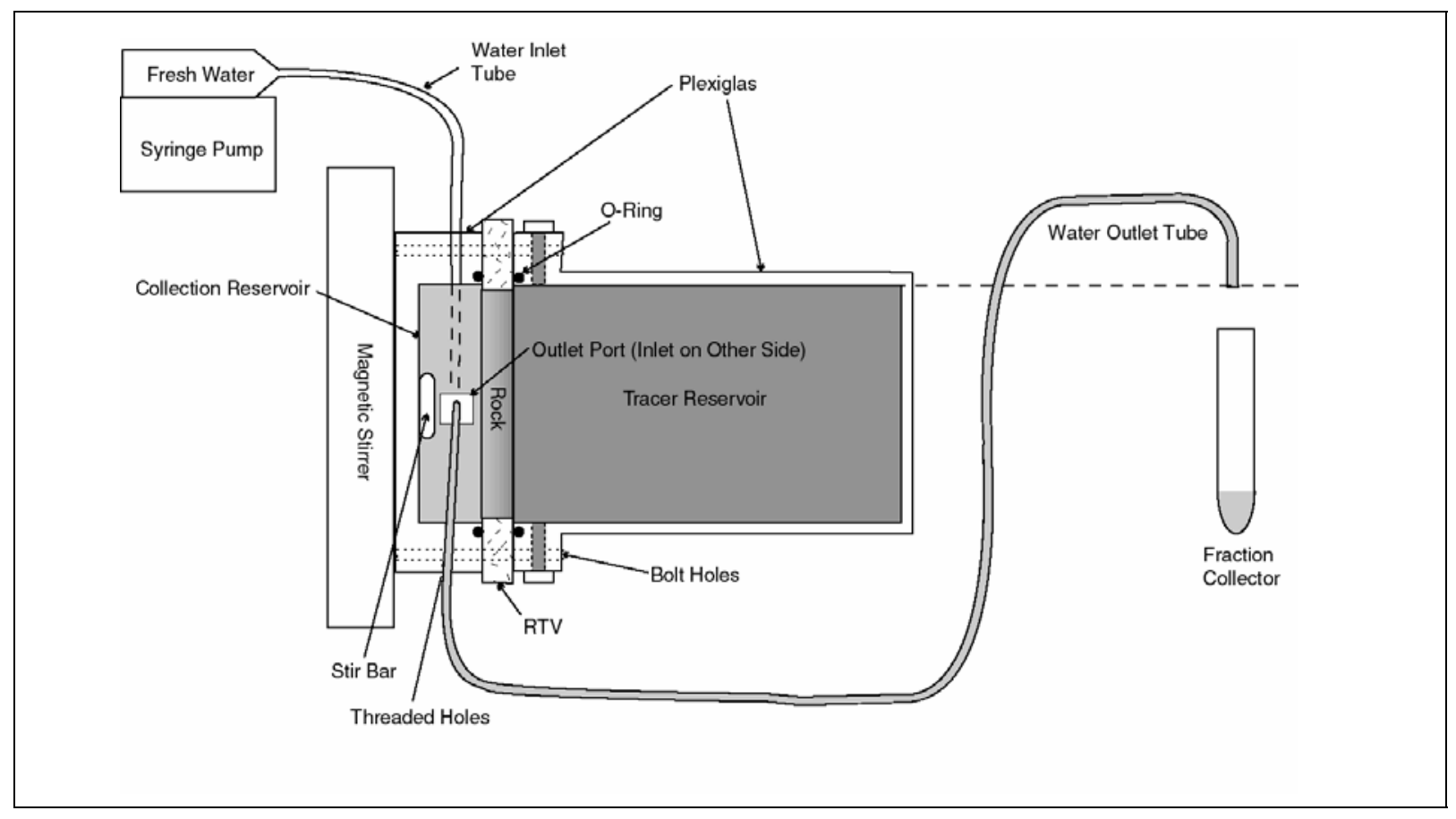

Schematic Illustration of a Diffusion Cell Experiment

these. When method (2) is used, it is better to have a smaller high-concentration reservoir than that depicted in Figure 2-1, and also a ready means of mixing and sampling this reservoir. Unless a very good independent estimate of the retardation factor in the porous medium is obtained, method (3) is the only method that will effectively work for a sorbing solute because the steady-state concentration profile is the only measurement that is independent of the retardation factor. Ideally, for a nonsorbing solute, measurements of concentrations in both reservoirs should be somewhat redundant and corroborative of one another, yielding the same diffusion coefficient estimate.

To obtain diffusion coefficient estimates in diffusion cell experiments, it is necessary to know (1) the porosity of the porous medium, (2) the exact dimensions of the porous medium (i.e., cross-sectional area and thickness in the direction of diffusion), and (3) the volumes of the two reservoirs. For consolidated geologic media, the dimensions of the sample can be controlled by cutting a core of known cross-sectional area perpendicular to its axis such that it has a fixed thickness. An estimate of the porosity of the sample can then be obtained by measuring the oven-dry and saturated weights of a sample and dividing the difference in these weights by the density of water times the known volume of the sample (see Attachment A of this document). For unconsolidated media, the sample must be contained in a sample "holder" of known dimensions that can be incorporated into the diffusion cell apparatus. Diffusion cell measurements of unconsolidated media are inherently more difficult and have more uncertainty than measurements of consolidated media because (1) unconsolidated media generally have higher permeability, making it is more difficult to avoid advective solute transport, and (2) the necessary (re)packing of an unconsolidated sample can be highly 
nonreprestentative of field conditions (i.e., the laboratory-packed samples are typically more porous and more permeable than under field conditions).

For perspective on diffusion coefficient estimates obtained from diffusion cell experiments, it is worthwhile to summarize the calculations necessary to estimate matrix diffusion coefficients in these experiments. First, it is assumed that solutes move according to one-dimensional diffusive transport through the porous medium. The one-dimensional diffusion equation is:

$$
\frac{\partial \mathrm{c}}{\partial \mathrm{t}}=\frac{D_{\mathrm{m}}}{\mathrm{R}_{\mathrm{m}}} \frac{\partial^{2} \mathrm{c}}{\partial \mathrm{x}^{2}}
$$

where

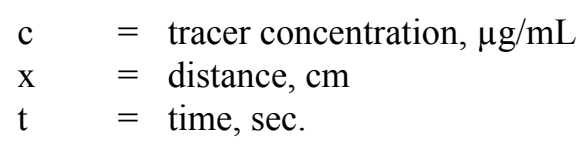

Although analytical solutions to this partial differential equation exist for simple boundary conditions (Carslaw and Jaeger, 1959), the time-dependent concentrations in the two diffusion cell reservoirs demand a numerical solution. The equations describing the tracer concentrations in the two reservoirs are, respectively:

$$
\begin{gathered}
\frac{\partial \mathrm{c}_{\mathrm{i}}}{\partial \mathrm{t}}=\left.\frac{\phi(S A)}{\mathrm{V}_{\mathrm{i}}} \frac{\partial \mathrm{c}}{\partial \mathrm{x}}\right|_{x=0} \\
\frac{\partial \mathrm{c}_{\mathrm{o}}}{\partial \mathrm{t}}=\left.\frac{\phi S A D_{\mathrm{m}}}{\mathrm{V}_{\mathrm{o}}} \frac{\partial \mathrm{c}}{\partial \mathrm{x}}\right|_{x=L}-\frac{\mathrm{Q}}{\mathrm{V}_{\mathrm{o}}} \mathrm{c}_{\mathrm{o}}
\end{gathered}
$$

where

$\mathrm{c}_{\mathrm{i}} \quad=$ solute concentration in inlet (high-concentration) reservoir, $\mu \mathrm{g} / \mathrm{mL}$

$\mathrm{c}_{\mathrm{o}}=$ solute concentration in outlet (initially solute-free) reservoir, $\mu \mathrm{g} / \mathrm{mL}$

$\mathrm{V}_{\mathrm{i}} \quad=$ volume of inlet reservoir, $\mathrm{mL}$

$\mathrm{V}_{\mathrm{o}} \quad=$ volume of outlet reservoir, $\mathrm{mL}$

$\mathrm{Q}=$ flush rate of outlet reservoir, $\mathrm{mL} / \mathrm{sec}$

$\phi \quad=$ porosity of matrix

$S A=$ cross-sectional area of porous medium, $\mathrm{cm}^{2}$

$\mathrm{L}=$ thickness of porous medium, $\mathrm{cm}$.

A key assumption in equations (B-12) and (B-13) is that both the inlet and outlet reservoirs are well mixed (i.e., they contain no internal concentration gradients). These equations must be modified somewhat if there is only discrete sampling of the outlet reservoir (i.e., no flushing) or if a very slow flushing/sampling rate is established for the inlet reservoir. Under steady-state conditions and with a very large inlet reservoir, all the time derivatives in equations (B-11-13) will be equal 
to zero, and the diffusion coefficient will be obtained exclusively from equation (B-13).

In this case, the concentration gradient $\left.\frac{\partial \mathrm{c}}{\partial \mathrm{x}}\right|_{x=L}$ is approximated by $\frac{\mathrm{C}_{\mathrm{i}}-\mathrm{C}_{\mathrm{o}}}{\mathrm{L}}$, and the retardation factor has no bearing on the estimate of the diffusion coefficient of a sorbing solute.

\section{Other Laboratory Methods of Measuring Matrix Diffusion Coefficients}

Most other laboratory methods of measuring or estimating matrix diffusion coefficients are variations of the diffusion cell experiment. A relatively common approach is to measure the decrease in solute concentration in a solution in contact with a known surface area and thickness of porous medium. In this case, there is no measurement of diffusion through the medium and the experiment is akin to measuring only the decrease in concentration in the high-concentration reservoir in a diffusion cell experiment. However, unlike a diffusion cell experiment, the porous medium has a no-flux boundary at some distance into the medium rather than an open boundary that solutes can pass through. For example, in Figure 2-1, the smaller reservoir would be replaced with an impermeable barrier at the interface between the sample and the small reservoir. Also, the large reservoir would be replaced with a much smaller one so that decreases in solute concentration would be easier to measure.

Another variation of the diffusion cell experiment is the "rock beaker" experiment in which a core of consolidated material is sub-cored along its main axis using a smaller diameter coring tool to form a "cup" in the sample (Triay et al., 1997). The large core is then encapsulated in epoxy or some other material that provides a no-flux boundary around its perimeter, and, after saturation of the core, a solution is introduced into the "cup" to induce diffusion in the radial direction into the matrix. Diffusion will also occur into the "bottom" of the cup as well. A variation of this method is to sub-core entirely through the larger core so that a "hole" is formed in the sample instead of a "cup." Solution can then be circulated through the hole to facilitate mixing and sampling and to avoid end effects, although this will be at the expense of additional experimental complexity. Either of these methods can be applied to more complicated geometries where cylindrical symmetry is not achieved (e.g., core holes drilled into rectangular or irregular-shaped blocks), but a more complex set of boundary conditions and possibly a 3-D solution to the diffusion equation will be required.

Diffusion coefficients of ions in porous media can also be inferred from electrical conductivity measurements (e.g., Conca and Wright, 1990). Solution conductance is related to ionic diffusion coefficients through the Nernst-Einstein equation (modified to account for porosity):

$$
D_{\mathrm{i}}=\frac{\mathrm{RT}}{\phi F^{2}} \frac{K \mathrm{t}_{\mathrm{i}}}{\mathrm{C}_{\mathrm{i}}}
$$


where

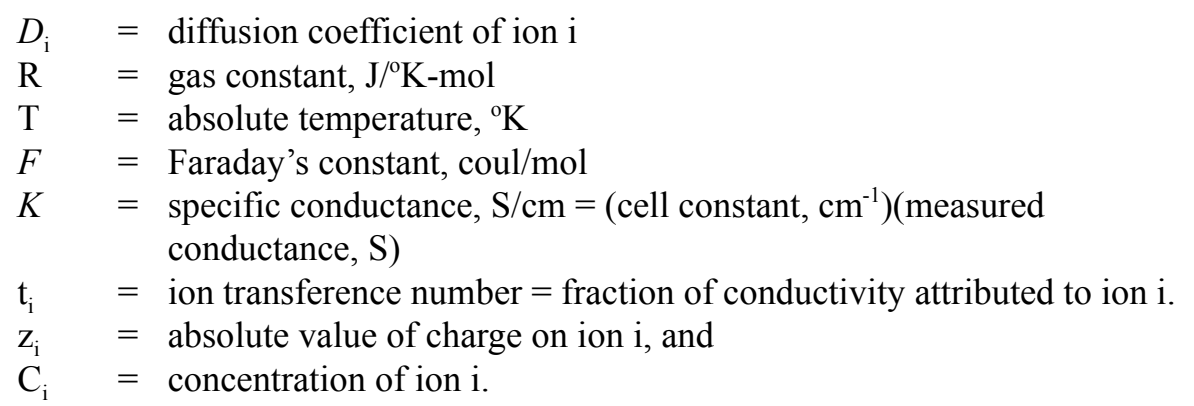

All of the terms in equation (B-14) are either known constants or are easily measurable, with the exception of ion transference numbers, $t_{i}$. Transference numbers for individual ions are dependent on concentrations of both the ion of interest and all other ions in solution. The transference numbers of all ions in a given solution must add up to one. Transference numbers for many ion pairs (a cation-anion pair that makes up a salt) are tabulated as a function of concentration in the literature (e.g., Robinson and Stokes, 1959). When literature values are unavailable for a given ion, multiple conductivity measurements must be made using the ion of interest with different counterions that have known ratios of transference numbers. This situation will apply to most complex actinide ions and many ions of rare fission products, as very few tabulated data exist for these ions.

The conductivity method of estimating diffusion coefficients is usually applied to bulk aqueous solutions, not porous media (e.g., Sato et al., 1996). Although in principle the method can be extended to porous media, significant complications arise in practice. Cation exchange in porous media can result in an unintended mixture of cations in solution, so the media must be carefully pre-equilibrated with the solution of interest to minimize such exchange and thus obtain meaningful and interpretable measurements. If alternating current (AC) measurements are conducted, which is often the case, solution impedance is measured instead of resistance, so the capacitive and inductive elements of impedance must be accounted for and effectively removed to obtain the true conductance of the solution. This correction is usually made by measuring impedance over a range of imposed frequencies and selecting the frequency where the phase angle shift between imposed and measured signals is smallest, which is the frequency at which the resistive component of impedance is largest relative to the capacitive and inductive components (Conca and Wright, 1990). However, even with these precautions, the use of equation (B-14) to translate conductance measurements into diffusion coefficients usually has relatively large errors associated with it. Perhaps the best use of conductance measurements is in obtaining relative diffusion coefficient measurements in porous media. For example, if a careful measurement is obtained for a given ion using one of the non-conductance methods described above, it should be relatively straightforward to apply the conductance method to obtain accurate diffusion coefficient estimates of other ions of the same charge. This can be done by measuring the relative conductance of simple salt solutions containing each ion of interest (including the one with the independent measurement) paired with a common counterion. 
More sophisticated electroanalytical methods exist for determining diffusion coefficients in free water. However, most of these are not widely applied. A good review of many of these methods is provided by Kariuki and Dewald (1996).

Recent advances in applying 2-D radiographic and 3-D tomographic imaging methods to hydrogeologic studies have opened up possibilities for more sophisticated methods of estimating diffusion coefficients in porous media. Diffusion-cell experiments and related measurement methods provide estimates of diffusion coefficients that are "averaged" over the domain of the porous media in the experiments. In reality, diffusion in porous media is likely to be multidimensional, with solute "fingering" in 2-D and 3-D along preferential diffusion pathways that are related to microstructural heterogeneities and internal variations in sample porosity and tortuosity.

Refined estimates of matrix diffusion coefficients can, in principle, be obtained by acquiring 2-D or 3-D images of solute diffusion "fronts" as a function of time in experiments that are otherwise quite similar to the experiments described above. $\mathrm{X}$-ray radiography and tomography have been used to obtain images of diffusion fronts of strong $\mathrm{x}$-ray absorbing elements, such as iodine. The iodide anion is commonly used because it seldom interacts chemically with geologic materials in groundwaters. However, one disadvantage of this approach is that it requires very high iodide (or other x-ray absorbing solute) concentrations to achieve good x-ray absorption contrast for quantitative imaging; these high concentrations can result in density-driven flow in the experiments if not properly designed. Neutron radiography and tomography also offer promise in obtaining diffusion coefficient estimates from 2-D and 3-D images of diffusion fronts. In this case, good image contrast can be achieved by ${ }^{2} \mathrm{H}_{2} \mathrm{O}$ diffusion into $\mathrm{H}_{2} \mathrm{O}$-occupied pore spaces or vice-versa. However, one must again be aware of density contrasts, as ${ }^{2} \mathrm{H}_{2} \mathrm{O}$ is approximately 1.1 times denser than $\mathrm{H}_{2} \mathrm{O}$. Problems with density contrasts in both $\mathrm{x}$-ray and neutron experiments can potentially be ameliorated by using concentrated salt solutions in place of $\mathrm{H}_{2} \mathrm{O}$, but then complex multicomponent diffusion will occur (also, the image contrast will decrease in the case of X-rays). Additionally, the resulting diffusion coefficient estimates will not necessarily be representative of diffusion under more dilute conditions (Newman, 1973).

Finally, both x-ray and neutron methods are best suited for high-porosity media. As porosity decreases, it becomes more difficult to obtain images of diffusion fronts because of smaller pore volumes and hence smaller numbers of x-ray or neutron absorbing atoms than can occupy pore spaces.

Imaging methods also offer computational challenges. Although radiographic and tomographic imaging computations are very well established, translating 2-D and 3-D images into diffusion coefficient estimates will require sophisticated algorithms and manipulations of large datasets that may involve averaging over cross-sectional areas or volume elements. Complex 3-D numerical computations may also be necessary.

Estimates of matrix diffusion parameters can also be obtained from column transport tests conducted in either fractures or unconsolidated porous media. These methods are discussed in the next section (under subheading "Variations in 
Tracer Testing Approaches") because they are conducted in much the same way as field tracer tests, which are discussed in detail in the next section.

\subsection{Field Measurements of Matrix Diffusion Parameters}

\subsubsection{Matrix Diffusion Mass Transfer Coefficients}

Diffusion coefficients cannot be measured directly in the field because it is not possible to control system geometry and boundary conditions to the extent necessary to make these measurements, at least not without considerable difficulty and expense. When tracer transport experiments are conducted in the field, lumped parameters such as the matrix diffusion mass transfer coefficient, $M T C_{D}=\frac{\phi}{b \eta} \sqrt{D_{\mathrm{m}} \mathrm{R}_{\mathrm{m}}}$, averaged over the domain of measurement, are obtained rather than individual parameters. Thus, an estimate of the diffusion coefficient, $D_{\mathrm{m}}$, can be obtained from field tests only if there are independent estimates of $\phi, b$, $\eta$, and $\mathrm{R}_{\mathrm{m}}$ (although $\mathrm{R}_{\mathrm{m}}$ can be assumed 1.0 for nonsorbing tracers). These estimates will all have considerable spatial variability and uncertainty associated with them, so any estimate of $D_{\mathrm{m}}$ will also have significant uncertainty. Although this may seem to be a serious limitation of field tests, the lumped mass transfer coefficient is actually more important than the individual parameters for predicting the effects of matrix diffusion over time scales that are shorter than

$T_{\dot{D}, \text { char }}=\frac{\mathrm{R}_{\mathrm{m}} L^{2}}{4 D_{m}}$ (see Section 1.2). Also, the scale of a field measurement is clearly

more appropriate for field scale modeling of contaminant transport than any laboratory-scale measurement. An independent estimate of $D_{\mathrm{m}}$ is really only needed to determine $T_{D, c h a r}$, which is essentially a measure of how long the overall groundwater travel time must be in fractures for a significant fraction of solute mass to be highly retarded by matrix diffusion (see Figure 1-1 and Figure 1-2).

\section{Cross-Hole Tracer Tests}

Field estimates of the $M T C_{D}$ are best obtained by conducting cross-hole tracer tests in which two nonsorbing tracers with different diffusion coefficients are simultaneously injected. The injection of two nonsorbing tracers with different diffusion coefficients makes it possible to separate the effects of hydrodynamic dispersion from matrix diffusion in the tracer responses. Both processes give rise to spreading of the solute breakthrough curves, including long tailing behavior, so it is not possible with only a single tracer to distinguish between the effects of dispersion and matrix. However, as a first approximation, differences in the breakthrough curves of two simultaneously-injected tracers with different diffusion coefficients can be attributed to differences in matrix diffusion. Conversely, the lack of any differences in tracer responses implies that matrix diffusion is not an operative process in the flow system (Becker and Shapiro, 2000). The simultaneous injection of tracers is important to ensure that the tracers follow the same flow pathways through the system and thus experience the same mean residence time and hydrodynamic dispersion in the aquifer. Figure 2-2 shows hypothetical cross-hole tracer responses for both a single-porosity system (with no matrix diffusion) and a dual-porosity system with matrix diffusion. Matrix diffusion causes the nonsorbing tracer with the larger diffusion coefficient 


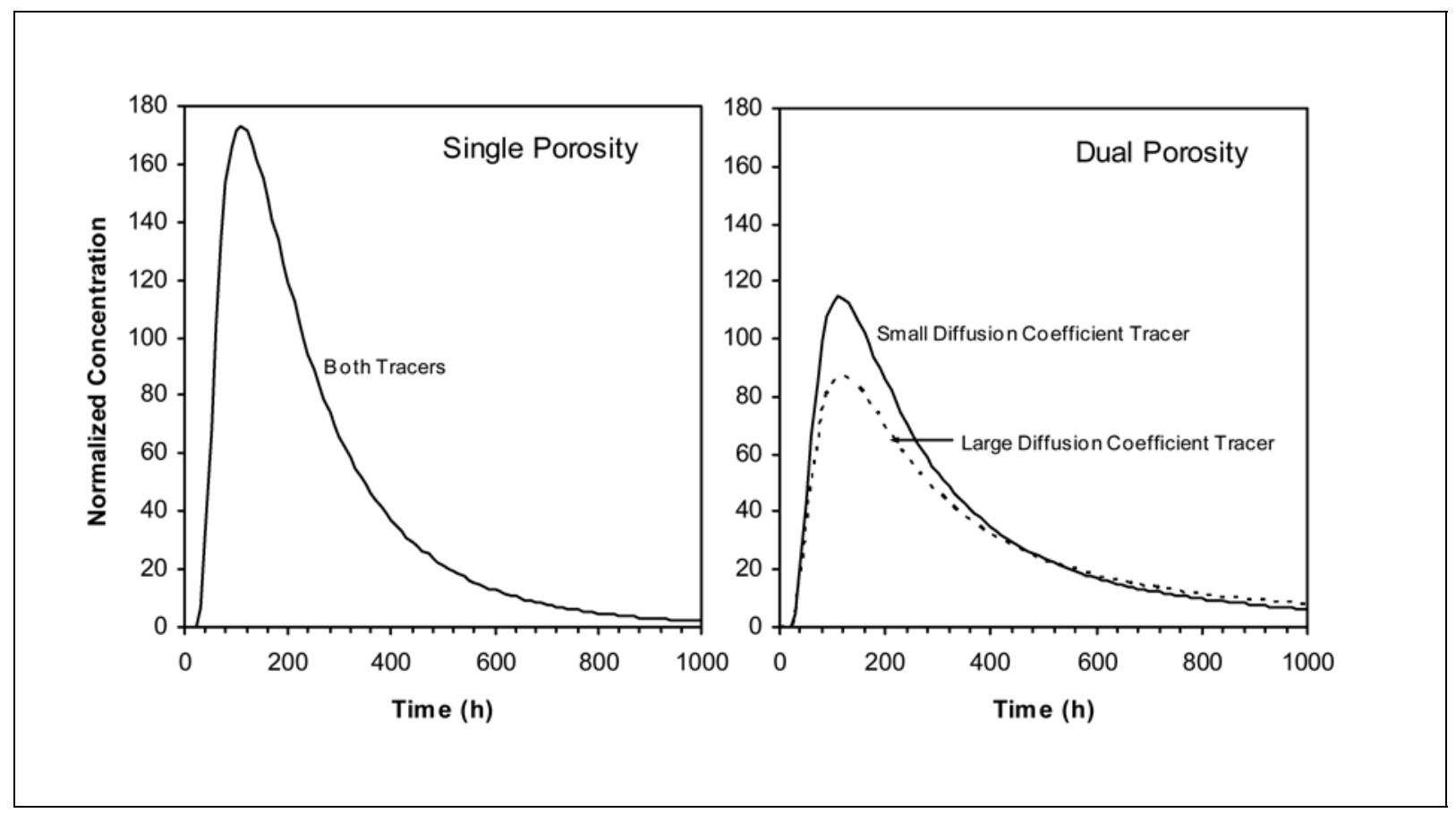

\section{Figure 2-2}

Hypothetical cross-hole tracer trest responses of nonsorbing solutes with different diffusion coefficients in a single-porosity system and a dual-porosity system. Curves generated using the RELAP computer model (Reimus and Haga, 1999)

to have a lower normalized peak concentration and longer tail than the tracer with the smaller diffusion coefficient. Note that when there is no matrix diffusion, the two nonsorbing tracers have identical responses.

To obtain quantitative estimates of $M T C_{D}$, the response curves in the right-hand plot of Figure 2-2 can be simultaneously fitted using the mean residence time, dispersion coefficient, and $M T C_{D}$ (and possibly the mass fraction of tracer participating in the test) as adjustable parameters. Because the tracers are injected simultaneously, all parameters except $M T C_{D}$ should be the same for the two tracers, and the values of $M T C_{D}$ for each tracer are constrained to have a ratio equal to the square root of the ratio of the tracer diffusion coefficients. The best-fitting values of $M T C_{D}$ should be quite well constrained because $M T C_{D}$ (with a fixed ratio for the two tracers) is the only adjustable parameter that can account for differences in the tracer responses. The primary requirement with this approach is that the ratio of tracer $D_{\mathrm{m}}$ values must be known reasonably well (the absolute $D_{\mathrm{m}}$ values are not critical).

\section{Single-Well Injection-Withdrawal Tracer Tests}

Single-well injection-withdrawal tracer tests involving two nonsorbing tracers with different diffusion coefficients can also be used to estimate $M T C_{D}$ values. In these tests, the tracers are simultaneously injected (generally followed by an injection of tracer-free "chase" water), typically allowed to "drift" in the aquifer for some period of time, and then they are pumped back out of the injection well. As with cross-hole tracer tests, $M T C_{D}$ values are estimated based on the 
differences in the tracer responses, rather than on individual breakthrough curves. Single-well tests offer the advantage of being less expensive (especially when well-drilling costs are considered), and they have durations that are more easily predicted than cross-hole tests. However, the scale of measurement in single-well tests (the volume of aquifer tested) is usually much smaller than in cross-hole tests.

\section{Variations in Tracer Testing Approaches}

Another approach to obtaining $M T C_{D}$ estimates in either single-well or cross-hole tracer tests is to conduct multiple tests in which all variables are kept the same (including tracer $D_{\mathrm{m}}$ values) except for the "drift" period in single-well tests or the production flow rate in cross-hole tests. Differences in the responses of tracers with the same $D_{\mathrm{m}}$ values as a function of residence time in the aquifer (assuming the tracers follow the same flow pathways for each residence time) can be used to obtain constrained estimates of $M T C_{D}$. The rationale for this approach is that $M T C_{D}$ is the only adjustable parameter that can account for differences in the tracer responses as a function of residence time. However, this approach is not as attractive as the multiple-tracer approach because the ambient groundwater flow, which is independent of tracer residence time, may cause tracers to follow slightly different flow pathways for different residence times, thus resulting in differences in dispersion as well as matrix diffusion. Also, it is more expensive to conduct two tests than one.

It should be noted that these tracer test approaches to obtaining estimates of $M T C_{D}$ are certainly not limited to field tests. The same approach can be taken in laboratory tests, with the advantage that independent measurements of $\phi, b$, and $\eta$ are much easier to obtain in laboratory-scale samples, so individual $D_{\mathrm{m}}$ values are more readily estimated. However, laboratory-scale tests have the disadvantage of being conducted at much less relevant scales than field tests. Also, fracture apertures and internal porosities in the laboratory are likely to be nonrepresentative of field conditions because lithostatic pressures, fracture offsets, and fracture filling materials present in the field are very difficult to reproduce in the laboratory.

\subsubsection{Flowing Fracture Spacing and Fracture Apertures}

Along with the matrix porosity, the average flowing fracture spacing, $L$, and the average fracture aperture, $2 b$, are the two most important parameters for determining the effective solute retardation factor caused by matrix diffusion, $1+\frac{\phi(L / 2)-b \eta}{b \eta}$ (for a nonsorbing solute). $L$ is also critical for determining an upper bound of $T_{D, \text { char }}$ for a given solute in a flow system. 


\section{High-Resolution Flow Surveys}

$L$ and $2 b$ cannot be estimated reliably in the laboratory, but rough estimates can be obtained in the field. The average spacing between discrete flowing intervals in high-resolution borehole flow surveys, preferably under pumped conditions, can be taken as a reasonable estimate of $L$. However, such estimates can be biased if flowing fractures or features are not oriented horizontally (perpendicular to the borehole). For instance, if two flowing fractures are $100 \mathrm{ft}$ apart in a vertical borehole, but they are both oriented at a $20^{\circ}$-angle relative to vertical, then the actual distance between the fractures will be $100 \sin \left(20^{\circ}\right)=34 \mathrm{ft}$. Televiewer or television borehole logs can be used to determine the orientations of fractures or fracture sets associated with flowing intervals to allow these types of corrections to be made. However, only fractures/features with significant flow should be considered when estimating $L$; it is likely that flowing fractures will be only a small subset of the fractures identified in televiewer or television logs. Also, it should be recognized that half the distance between flowing fractures/features is only an upper bound estimate of the distance that solutes can diffuse into the matrix before encountering a no-flux boundary. The effective diffusion distance could be considerably less than $L / 2$ if there are diffusion boundaries such as fractures filled with impermeable material or large increases in matrix tortuosity at distances less than $L / 2$ into the matrix.

\section{Inferences from Tracer Testing}

Small effective diffusion distances might be detected in field tracer tests as deviations from the expected relative breakthrough curves of two nonsorbing tracers with different diffusion coefficients, particularly in long-duration tests. Figure 2-3 shows how the relative breakthrough curves of two nonsorbing tracers with different diffusion coefficients change in a cross-hole tracer test when a diffusion boundary is encountered vs. when no diffusion boundary is encountered (i.e., when the matrix acts as if it is essentially infinite over the time scale of the test). Clearly, these differences may be subtle and difficult to detect if there are small signal-to-noise ratios or background interferences because most of the difference is seen in the low-concentration tails of the response curves.

These differences can also be looked for in single-well injection-withdrawal tracer tests. Indeed, if determining the effective diffusion distance into the matrix is a primary objective, single-well tests offer an advantage over cross-hole tests in that tracer residence times in the aquifer can be much more easily and inexpensively controlled (the tracers can be allowed to sit in the aquifer for an extended period of time without equipment or labor required on site). However, excessive times may be required to estimate the effective diffusion distance into the matrix if the distance is more than a few 10 s of centimeters. As a first approximation, the distance that a solute diffuses into the matrix as a function of time $t$ is equal to $\sqrt{2 D_{\mathrm{m}} \mathrm{t}}$. Assuming a matrix diffusion coefficient of $10^{-5} \mathrm{~cm}^{2} / \mathrm{sec}$, which is really more typical of a free-water diffusion coefficient, the distance that a solute will diffuse in one year will be $\sim 25 \mathrm{~cm}$, and in five years it will be $\sim 56 \mathrm{~cm}$. Thus, diffusion distances of more than half a meter will be impractical to measure by tracer test methods. However, field tracer methods still have merit because there is always the possibility of relatively short diffusion distances into the matrix, which cannot be measured by any other method. If such small diffusion distances 


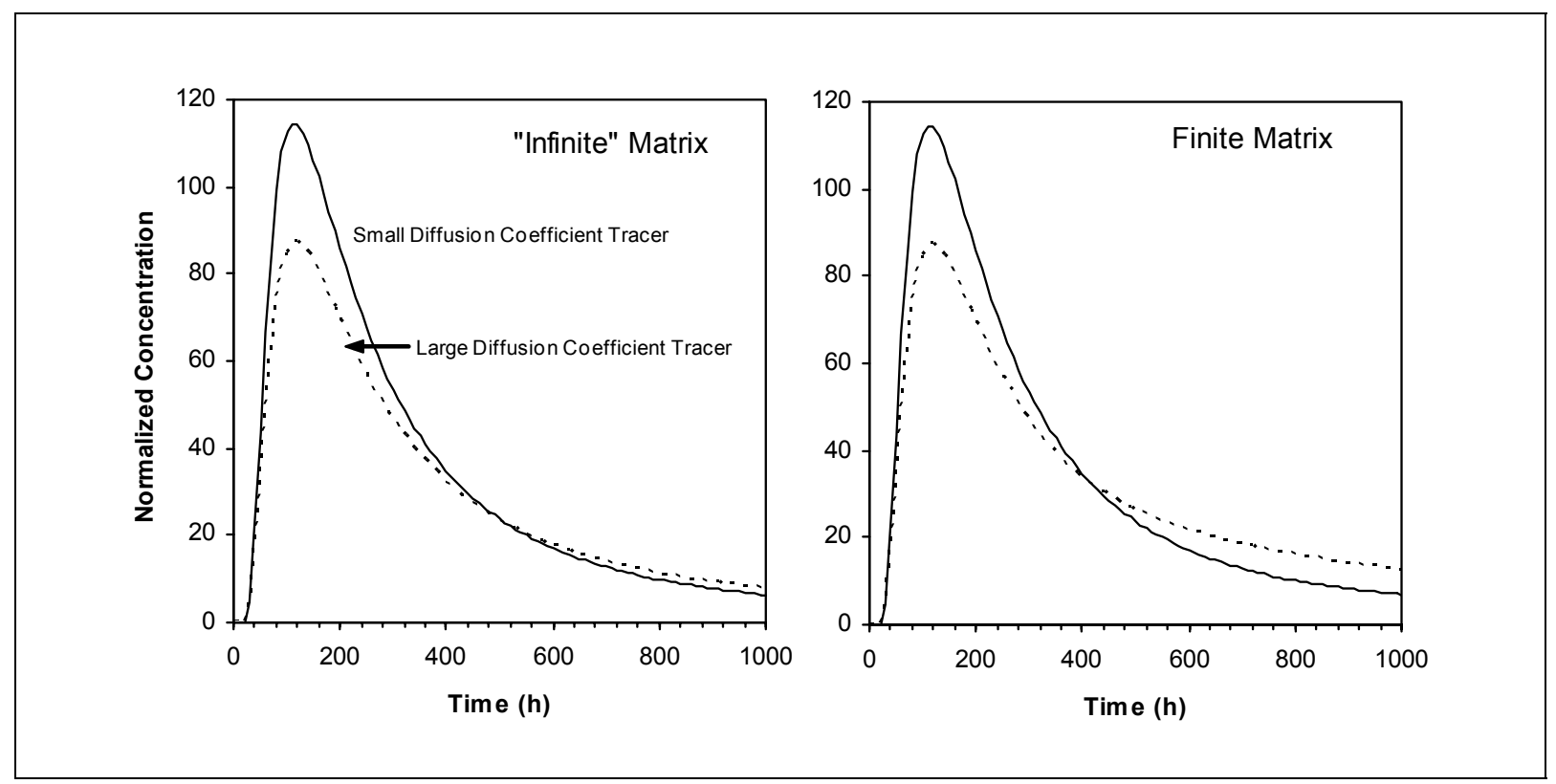

Figure 2-3

Differences in multiple nonsorbing tracer responses in cross-hole tracer test with an "infinite" matrix (no diffusion boundary) and a finite matrix (diffusion boundary). Note the greater difference in the tail concentrations of the two tracers for the finite matrix. Curves generated using the RELAP computer model (Reimus and Haga, 1999)

are measured, the implications for CAU-scale predictive modeling are enormous, as estimates of retardation factors accounting for matrix diffusion might be reduced by over an order of magnitude relative to estimates based on flowing fracture spacing.

Estimates of average fracture half-apertures, $b$, can be obtained from field tracer tests provided independent estimates of $\phi$ and $D_{\mathrm{m}}$ are obtained from laboratory measurements on cores taken from the same interval in which the field test(s) is/are conducted. The value of $M T C_{D}=\frac{\phi}{b \eta} \sqrt{D_{\mathrm{m}} \mathrm{R}_{\mathrm{m}}}$ derived from a cross-hole, multiple-tracer test can be inverted and multiplied by $\phi \sqrt{D_{\mathrm{m}}}$ to obtain an estimate of $b$ (assuming a nonsorbing tracer with $\mathrm{R}_{\mathrm{m}}=1$, and also assuming $\eta=1$ ). This estimate of $b$ should be superior to any estimate based on hydraulic conductivity measurements (i.e., a hydraulic aperture) or to estimates based on measuring apertures of fractures in cores or borehole television logs.

If estimates of effective flow porosity are obtained from cross-hole tracer tests (see Attachment A, Porosity Data), they can be used to obtain crude estimates of the effective retardation factor caused by matrix diffusion, $\mathrm{R}_{M D}$ with $\mathrm{R}_{\mathrm{f}}=\mathrm{R}_{\mathrm{m}}=1$, without having direct estimates of $L$ or $b$. As discussed in Section 1.2, $\mathrm{R}_{\mathrm{MD}}$ for a nonsorbing solute is one plus the ratio of stagnant water to flowing water, so estimates of matrix porosity and flow porosity can be combined to estimate $\mathrm{R}_{\mathrm{MD}}$ for a nonsorbing solute as follows:

$$
\mathrm{R}_{\mathrm{MD}}=1+\frac{\phi(1-\varepsilon)}{\varepsilon}
$$


where

$\varepsilon \quad=\quad$ flow porosity determined from cross-hole tracer test.

Equation (B-15) is an upper bound estimate of $\mathrm{R}_{\mathrm{MD}}$ for a nonsorbing solute because it assumes that the entire matrix is accessible to solutes by diffusion. If the cross-hole tracer test also provides an estimate of $L$, as discussed above, and flowing fracture spacing information is available from borehole flow surveys (and televiewer or television logs), then a refined estimate of $\mathrm{R}_{\mathrm{MD}}$ for a nonsorbing solute that does not require an estimate of $b$ can be obtained as follows:

$$
\mathrm{R}_{\mathrm{MD}}=1+\frac{L}{S} \frac{\phi(1-\varepsilon)}{\varepsilon}
$$

where

$S \quad=$ flowing fracture spacing, $\mathrm{cm}$

However, it should be noted that estimates of effective flow porosity, $\varepsilon$, from cross-hole tracer tests are often considered very uncertain, and they have a tendency to overpredict true flow porosity (Reimus, 2003). Thus, $\mathrm{R}_{\mathrm{MD}}$ estimates for nonsorbing solutes based on equations (B-15) or (B-16) should be considered quite uncertain (although they will tend to be conservative if $\varepsilon$ is overpredicted).

\subsubsection{Insights from Geochemical and Isotopic Analyses of Groundwaters}

Geochemical and isotopic analyses of groundwaters could potentially yield valuable insights into effective matrix diffusion parameters (both mass transfer and geometric parameters) over larger scales than any other methods discussed in this attachment. Differences (or lack thereof) in water chemistry at various locations or between groundwater and recharge water or other inputs have commonly been used to infer groundwater flow pathways and travel times. Such information along a known or suspected flow pathway could, in principle, also be used to constrain matrix diffusion parameters in coupled flow and transport models. Maloszewski and Zuber (1991) showed how to correct apparent ${ }^{14} \mathrm{C}$ groundwater ages for the effects of matrix diffusion, and it is certainly plausible that the inverse of this approach could be taken to obtain estimates of matrix diffusion parameters from geochemistry data.

Uncertainties associated with such an approach would have to be evaluated on a case-by-case basis, as they would be a function of the quantity, quality, and spatial distribution of geochemical data from a flow system, as well as the existing knowledge of the nongeochemical characteristics of the system hydrogeology. However, even if only relatively broad ranges of matrix diffusion parameters were identified that were either consistent or inconsistent with geochemical and isotopic groundwater data, this information would have considerable value in constraining CAU-scale transport predictions. Also, such analyses could contribute significantly to the validation and corroboration of CAU-scale flow and transport 
models. Innovative experimental methods, such as isolated sampling of adjacent high and low flow zones (nominally, fracture and matrix water, respectively) in wells with open completions, could provide valuable constraints for interpretations of datasets and corresponding uncertainty reduction.

\subsection{Summary of Measurement Methods}

Laboratory methods are most appropriate for measuring the matrix diffusion coefficient, $D_{\mathrm{m}}$. These measurements generally require a separate measurement of $\phi$, which is also an important parameter that affects matrix diffusion. Laboratory methods cannot be used to effectively obtain estimates of any of the three major lumped parameters that govern matrix diffusion, although tracer transport experiments in laboratory-scale systems can provide estimates of $M T C_{D}$.

Field tracer tests are appropriate for obtaining estimates of $M T C_{D}$. However, they do not yield direct estimates of $D_{\mathrm{m}}, \phi$, or any of the other individual parameters that make up the mass transfer coefficient.

Estimates of the average flowing fracture spacing, $L$, can be obtained from borehole flow surveys, preferably combined with televiewer or television logs to determine orientation of flowing fractures. However, these estimates should be considered upper bounds of the effective distance that a solute can diffuse into the matrix. Long-duration tracer tests may provide better (more conservative) estimates of effective solute diffusion distances into the matrix, although significant uncertainties and expenses may be involved.

Estimates of average fracture half-apertures, $b$, can be obtained from field tracer tests provided independent estimates of $\phi$ and $D_{\mathrm{m}}$ are obtained from laboratory measurements on cores taken from the same interval in which the field test(s) is/are conducted.

If effective flow porosity estimates are available from cross-hole tracer tests, then effective retardation factors caused by matrix diffusion (for nonsorbing solutes) can be estimated directly from matrix porosity and flow porosity using equation (B-15) (or equation B-16 if estimates of $L$ and flowing fracture spacing, $S$, are also available). However, these estimates should be considered quite uncertain because of the tendency for cross-hole tracer tests to overpredict flow porosity.

More work is needed before matrix diffusion parameter estimates derived from geochemical and isotopic groundwater data can be accepted with a high degree of confidence. 


\section{0}

\section{Considerations for Data Transferability}

When evaluating matrix diffusion coefficient data for transferability to a NTS CAU, several factors must be considered. These factors are discussed in this section. The last subsection of this section addresses adjustments that should be made to matrix diffusion coefficient data to account for differences in solute characteristics, temperature, and ionic strength between transferred datasets and radionuclides and conditions of interest within CAUs. These adjustments do not necessarily affect the transferability of the diffusion coefficient data, but they are important when using the data in CAU-scale transport modeling.

\subsection{Importance of Data in CAU-Scale Transport Modeling}

Perhaps the biggest consideration in the transferability of matrix diffusion data is not any technical factor associated with the appropriateness of data transfer, but rather a consideration of which matrix diffusion data, for a given CAU, are important in CAU-scale modeling and which data are really not important. This determination will be CAU specific and it will involve some type of sensitivity analysis using the best available information, which implies some degree of knowledge of the system as well as some degree of uncertainty.

The three lumped parameters summarized in Section $1.3\left(\mathrm{R}_{\mathrm{MD}}, T_{D, c h a r}\right.$ and $\left.M T C_{D}\right)$ are critical to this determination because they are ultimately the parameters that quantify the effect of matrix diffusion in a given CAU. In some cases, it may be easier to measure or transfer these lumped parameters than the individual parameters that comprise them. A sensitivity analysis to determine the relative importance of matrix diffusion data should proceed as follows.

If groundwater travel times in flowing porosity in a given CAU are predicted to be long enough (with reasonable certainty) that they greatly exceed $T_{D, c h a r}$ for any reasonable choice of $L$ and $D_{\mathrm{m}}$, then the effective retardation factor for matrix diffusion will describe transport in the system. In this case, transferring $D_{\mathrm{m}}$ values will really not be very important for CAU-scale transport modeling, but $\phi, L$ and $b$ values will be very important because they define the effective retardation factor. Conversely, if groundwater travel times in flowing porosity appear to be very rapid relative to reasonable estimates of characteristic matrix diffusion times, it will be very important to transfer (or conduct CAU-specific tests to obtain estimates of) $M T C_{D}$ values. In this case, estimates of the individual parameters $L$, $b$, and $D_{\mathrm{m}}$ will be less important than $M T C_{D}$ values in determining CAU boundaries. If there is simply not enough information available (or the information is too uncertain) to determine which of the above cases apply, or if it appears that neither of these extremes will apply, then it is prudent to proceed with data transfer and/or collection of $L, b$, and $D_{\mathrm{m}}$ values. 
This approach carries some risk in that there may be biased or incomplete information that goes into the sensitivity analyses, which could result in an incorrect assessment of parameter importance. Nevertheless, in most cases, such an up-front assessment should help in focusing efforts for data transfer and collection.

\subsection{Dependence of Matrix Diffusion Coefficients on Matrix Porosity and Permeability}

It is quite well known that diffusion coefficients in porous media tend to be positively correlated with both the porosity and permeability of the media (e.g., Boving and Grathwohl, 2001). Furthermore, diffusion coefficients tend to be more strongly correlated with permeability than porosity. Reimus et al. (2002) obtained the following regression equation for matrix diffusion coefficients of ${ }^{3} \mathrm{HHO}, \mathrm{Br}^{-}$, and I' (combined) in saturated volcanic rock matrices from the NTS:

$$
\log \left(D_{\mathrm{m}}\right), \mathrm{cm}^{2} / \mathrm{s}=-3.38+1.48(\phi)+0.174\left(\log \text { Perm }, \mathrm{m}^{2}\right)
$$

The 95 percent confidence intervals of the regression parameters are (Draper and Smith, 1981):

$$
\begin{gathered}
\text { Intercept }=-3.38 \pm 1.55 \\
\text { Coefficient for porosity }=1.48 \pm 1.27 \\
\text { Coefficient for log perm. }=0.174 \pm 0.084 .
\end{gathered}
$$

This regression equation is based on 40 different diffusion cell measurements, some of which were replicates. The larger 95 percent confidence interval (relative to the mean) for the porosity coefficient compared with the log permeability coefficient is consistent with an F-test result that log permeability is a better predictor variable for the matrix diffusion coefficient than matrix porosity. However, both variables were found to be significant additions to the regression equation (after the other variable was already in the equation) at the 95 percent confidence level. Note that Reimus et al. (2002) (equation 2.5) report slightly different regression coefficients for porosity and $\log$ permeability and a slightly different intercept from equation (B-17) because a few of the data points were inadvertently weighted by a factor of 2 in Reimus et al. (2002).

The fact that matrix diffusion coefficients are more strongly correlated with matrix permeability than porosity is intuitively explained by recognizing that permeability is really a measure of the interconnectedness and limiting pore throat sizes within the matrix porosity. Interconnectedness and pore throat sizes are the key controlling factors for solute matrix diffusion as well. A rock with a relatively large porosity that is very poorly connected and has essentially zero permeability will also have a matrix diffusion coefficient close to zero because if there are no pathways for flow through the rock, there will be no pathways for diffusion either. In fact, although porosity is retained in the above regression equation at the 95 percent confidence level, some of its ability to predict matrix diffusion coefficients might be attributable to its positive correlation with log permeability.

It must be emphasized in this section that the dependence of matrix diffusion coefficients is on matrix porosity and matrix permeability, not on bulk porosity or 
bulk permeability, which are the parameters generally obtained from aquifer tests or field tracer tests. Measurements of these bulk parameters should usually not be considered when assessing transferability of matrix diffusion data.

\subsection{Dependence of Matrix Diffusion Coefficients on the Geologic Setting}

It seems intuitively logical, and it is very tempting to conclude, that matrix diffusion coefficients are highly dependent on geologic setting, which includes factors such as rock type, depositional history, alteration history, and structural setting. However, it is probably the underlying dependence that porosity and permeability have on these geologic factors that dictates the apparent dependence of matrix diffusion coefficients on geologic settings rather than the settings themselves. Thus, geologic setting should not be a heavily-weighted consideration in assessing the transferability of matrix diffusion coefficient data, particularly if matrix porosity and permeability data are available for the location being considered. Geologic setting should be used only as a "backup" factor for assessing data transferability if matrix porosity and permeability data are not available. The dependence of matrix porosity on geologic setting is discussed in detail in Attachment A, Porosity Data.

\subsection{Dependence of Other Matrix Diffusion Parameters on the Geologic Setting}

The average flowing fracture spacing, $L$, and average fracture apertures, $2 b$, will depend strongly on the geologic setting, and in particular on the type of rock and the stress and strain history within the rock. In general, competent or brittle rocks (e.g., granites, welded tuffs, carbonates) tend to fracture more readily under stress than less-competent or ductile rocks (e.g., nonwelded tuffs, sandstone).

Less-competent rocks will tend to deform rather than fracture under stress (Wilson et al., submitted). Thus, all other things being equal, competent rocks will tend to have smaller values of $L$ than less-competent rocks. Competent rocks should also tend to have larger values of $2 b$ than less-competent rocks, because competent rocks have more strength to keep fractures propped open when strain causes offsets of fracture surfaces (Durham and Bonner, 1994).

It must be emphasized that these are only broad generalities that can be readily negated by many factors. Large fracture densities do not necessarily imply small values of $L$ because it is the spacing of flowing fractures that dictates $L$, not the spacing of all fractures. Local structural setting can also have a significant influence on $L$ and $b$. A nonwelded tuff in a fault zone may have much smaller values of $L$ and larger values of $b$ than a welded tuff that has experienced minimal stress and strain. Great care should be taken when transferring $L$ and $b$ data from one location to another because of these factors. Care should also be taken when transferring $\mathrm{R}_{\mathrm{MD}}$ values (effective retardation factors accounting for matrix diffusion) derived from effective flow porosity estimates in cross-hole tracer tests because effective flow porosity estimates are generally quite uncertain and location specific. 


\subsection{Type of Measurement}

Another consideration in transferring matrix diffusion data from other locations to NTS CAUs is the method of measurement. As discussed in Section 2.0 (and summarized in Section 2.3) different measurement methods yield data with different uncertainties and scales of relevance. The following rules of thumb apply given current measurement technologies.

\section{$D_{m}$ Estimates}

For $D_{\mathrm{m}}$ values, the best estimates are obtained from laboratory diffusion-cell experiments, or any method that involves measuring diffusion through or into a rock matrix based on monitoring solute concentration increases or decreases on either side of the sample. Slightly more weight should probably be given to an experiment in which both concentration increases and decreases are measured because this essentially amounts to making two measurements that are averaged or confirmatory. More weight should also be given to experiments in which the diffusion distance is longer because larger-scale experiments are less likely to be influenced by small-scale heterogeneities that are unimportant at field scales. Samples that include natural fracture surfaces are desirable to include in any diffusion cell test matrix, as the natural surfaces may have mineral coatings or other subtle alterations that could affect diffusion.

In this regard, it is also desirable to obtain estimates of $D_{\mathrm{m}}$ values from laboratory-scale transport experiments in natural fractures. Such experiments are conducted and interpreted in exactly the same way as cross-hole tracer tests in the field (see Section 2.3 - either simultaneously inject two nonsorbing tracers with different diffusion coefficients, or conduct multiple tests with tracers of the same diffusion coefficient at different flow rates). However, laboratory fracture transport experiments are often of much shorter duration than diffusion cell experiments, so solutes may actually interrogate a shorter distance into the matrix than in a diffusion cell experiment. Also, as with field-scale tracer tests, $D_{\mathrm{m}}$ values are not measured directly in fracture transport experiments but must be inferred from matrix diffusion mass transfer coefficients, so an estimate of $b$ must also be obtained, which introduces additional uncertainty into the measurement. Both diffusion cell methods (and related approaches) and fracture transport experiments require independent estimates of $\phi$ to obtain estimates of $D_{\mathrm{m}}$.

$D_{\mathrm{m}}$ values obtained from conductivity measurements are not as reliable as diffusion cell experiments (or related method) because they are indirect measurements that rely on assumptions or additional measurements that may have considerable uncertainty. However, the conductivity method can provide good relative estimates of diffusion coefficients, which can then be translated into to good absolute estimates if one or more measurements are effectively "calibrated" with a diffusion cell measurement.

$\mathrm{X}$-ray and neutron imaging methods hold great promise for achieving refined estimates of $D_{\mathrm{m}}$ values that include within-sample variability. However, these methods are relatively immature and expensive, and they have technical challenges, such as eliminating or accounting for solution density contrasts. Measurements using these methods should be evaluated for transferability on a 
case-by-case basis, although in general they should be given no more weight than a diffusion cell or related method of measurement.

\section{$M T C_{D}$ Estimates}

Estimates of matrix diffusion mass transfer coefficients, $M T C_{D}$, are best obtained in field tracer tests using multiple nonsorbing tracers with different diffusion coefficients. Longer-duration and larger-scale tests will provide more representative estimates for CAU-scale transport modeling than shorter-duration or smaller-scale tests. Cross-hole tracer tests are generally preferred over single-well tests because they usually interrogate a larger volume of the flow system. However, single-well tests with very long "drift" periods can have greater solute diffusion distances into the matrix than cross-hole tests of shorter duration, which is advantageous for determining if matrix diffusion coefficients tend to decrease as diffusion distances increase or if a diffusion boundary is encountered in the matrix. Estimates of $M T C_{D}$ from laboratory tests should not be weighted as heavily as estimates from field tests because of their smaller scale and also because of the difficulty and uncertainty associated with reproducing ambient fracture apertures in the laboratory.

\section{Flowing Interval Spacing (L) Estimates}

Upper bound estimates of $L$ are best obtained from borehole flow surveys with corresponding televiewer or television logs to determine orientations of flowing fractures or features. Estimates of $L$ from flow survey information alone should not be weighted as highly as flow survey estimates corrected for fracture orientations. $L$ should not be estimated from fracture statistics alone because frequently only a small subset of fractures contribute to flow.

More conservative estimates of $L$ can potentially be obtained from field tracer tests involving multiple nonsorbing tracers with different diffusion coefficients. If the normalized tracer responses in the tests deviate significantly from the expected behavior in a system with an "infinite" matrix, then a reasonable estimate of $L$ may be obtained that is likely to be much smaller than estimates from flow surveys. Longer duration tracer tests stand a better chance of obtaining estimates of $L$ than shorter duration tests.

\section{Fracture Half-Aperture (b) Estimates}

Estimates of fracture half-apertures, $b$, are best obtained from field tracer tests using independently-obtained estimates of $\phi$ and $D_{\mathrm{m}}$ in conjunction with estimates of $M T C_{D}$. Estimates of $b$ can be "backed out" by inverting $\frac{\phi}{b \eta} \sqrt{D_{\mathrm{m}} \mathrm{R}_{\mathrm{m}}}\left(=M T C_{D}\right)$ and multiplying by $\phi \sqrt{D_{\mathrm{m}}}$ (assuming a nonsorbing tracer with $\mathrm{R}_{\mathrm{m}}=1$, and also assuming $\eta=1$ ). For the purposes of transport modeling, estimates of $b$ obtained this way will be far superior and should be weighted much more than estimates based on hydraulic data or on fracture apertures measured in core samples or in borehole television logs. 


\subsection{Data Reduction and Analysis Method}

Even when the best possible measurement method is used, the methods and quality of data reduction and analyses must be considered when transferring matrix diffusion data. Poor data reduction or poor interpretive methods can reduce the confidence in, or increase the uncertainty of, an otherwise sound dataset. In many cases, it may be possible to re-analyze the data using better methods. However, when this is not possible, it may be necessary to place less emphasis on the dataset than if the data reduction and analyses methods were optimal.

\subsection{Quality of Documentation}

Quality of documentation is also a factor in determining the transferability of matrix diffusion data. Poor documentation should reduce the relative value placed on a given dataset, and in extreme cases, it might even disqualify a dataset from being transferred. The documentation quality factor should not be confused with the data reduction and analysis method factor. A high-quality dataset and interpretative analysis can sometimes be very poorly documented. However, it is often the case that a poor analysis is also poorly documented, and in some cases, it may be difficult to distinguish between a poor analysis and poor documentation.

\subsection{Dependence of Matrix Diffusion Coefficients on Solute Characteristics, Temperature, and lonic Strength}

Although solute characteristics, temperature, and ionic strength do not directly influence the transferability of matrix diffusion data, they can dictate how the transferred data should be adjusted to better represent the radionuclides and conditions of interest within NTS CAUs. This section addresses such adjustments.

Equation (B-17) provides estimates of matrix diffusion coefficients for ${ }^{3} \mathrm{HHO}$ and simple anions (halides) in volcanic rock matrices at $\sim 20-25^{\circ} \mathrm{C}$ in waters with an ionic strength of about $0.0035 \mathrm{M}$ (typical of Pahute Mesa groundwaters) as a function of matrix porosity and permeability. For other solutes, different temperatures, or different ionic strengths, it may be necessary to adjust these diffusion coefficient estimates before they are used in predictive models. Also, when transferring data obtained for a given solute at a given temperature and ionic strength from another location, it may be necessary to make adjustments to the data before they are used in predictive models of radionuclide transport at CAU-relevant temperatures and ionic strengths. However, in general, the variability in matrix diffusion coefficients as a result of variability in porosity and permeability should be much greater than the variability caused by species properties, temperature, and ionic strength.

Empirical correlations exist in the literature to adjust free diffusion coefficients for species size and charge, but, for matrix diffusion coefficients, it is more convenient to simply provide general guidance for such adjustments. As a general rule, cations have smaller diffusion coefficients than anions because they tend to be more hydrated (i.e., more water molecules move with them, giving them a larger effective radius). Most simple monovalent cations (e.g., $\mathrm{K}^{+}, \mathrm{NH}^{+}, \mathrm{Cs}^{+}$) have diffusion coefficients only 5 to 10 percent smaller than simple monovalent 
anions such as $\mathrm{Br}^{-}$and $\mathrm{I}^{-}$(Newman, 1973). $\mathrm{PuO}_{2}^{+}$and $\mathrm{NpO}_{2}^{+}$probably have diffusion coefficients of this magnitude because they both have relatively low charge to mass ratios and should not be highly hydrated. However, cations with high charge to mass ratios have much smaller diffusion coefficients. For instance $\mathrm{Na}^{+}$has a diffusion coefficient about 0.65 times that of $\mathrm{Br}^{-}$and $\mathrm{I}^{-}$, and $\mathrm{Li}^{+}$has a diffusion coefficient about 0.5 times that of these anions (Newman, 1973). Multivalent cations also tend to have small diffusion coefficients, ranging from 0.3 to 0.4 times that of $\mathrm{Br}^{-}$and $\mathrm{I}^{-}$(Newman, 1973). Cations with charges of +3 or more typically hydrolyze or form complexes in solution to become species of lower charge (e.g., hydroxyl or carbonate complexes). Of course, it must be remembered that cations will also tend to sorb by cation exchange or surface complexation in most rock matrices; the above discussion applies only to diffusion, not sorption.

Large monovalent anions, such as pentafluorobenzoate, have diffusion coefficients about 0.33 times that of $\mathrm{Br}^{-}$and $\mathrm{I}^{-}$(Callahan et al., 2000). The diffusion coefficients of such weak organic bases can be considered reasonable lower bounds for diffusion coefficients of large anionic radionuclide complexes. Multivalent anions (which are generally multi-atom species) tend to have diffusion coefficients of 0.4 to 0.6 times that of $\mathrm{Br}^{-}$and $\mathrm{I}^{-}$.

The Stokes-Einstein equation predicts that diffusion coefficients will be directly proportional to absolute temperature and inversely proportional to fluid viscosity (which decreases as temperature increases, but not linearly). Table 3-1 summarizes the factor by which diffusion coefficients should increase (relative to $20^{\circ} \mathrm{C}$ in pure water) as a function of temperature (Weast and Astle, 1982). The higher temperatures in Table 3-1 will be relevant to near-cavity conditions for some time after re-saturation in CAU transport modeling.

Diffusion coefficients are a relatively weak function of ionic strength until ionic strengths become greater than about $1 \mathrm{M}$ (Newman, 1973). From infinite dilution to $1 \mathrm{M}$ ionic strength, diffusion coefficients should change no more than \pm 10 percent. If ionic strengths exceed $1 \mathrm{M}$, diffusion coefficients may increase or decrease by as much as 25 percent from their values at infinite dilution. Ionic strengths greater than $1 \mathrm{M}$ are unlikely to occur at NTS, even in cavities, so the effect of ionic strength on diffusion coefficients is considered to be relatively minor compared to the effect of rock properties and temperature. However, if data are transferred from locations where ionic strengths exceed $1 \mathrm{M}$, then appropriate adjustments should be made for CAU-specific conditions. 
Table 3-1

Factor by Which Diffusion Coefficients Change as a Function of Temperature in Water

\begin{tabular}{|c|c|}
\hline Temperature, $^{\circ} \mathbf{C}$ & Relative Diff. Coef. \\
\hline \hline 20 & 1.0 \\
\hline 25 & 1.14 \\
\hline 30 & 1.30 \\
\hline 40 & 1.64 \\
\hline 50 & 2.14 \\
\hline 60 & 2.58 \\
\hline 70 & 3.07 \\
\hline 80 & 3.59 \\
\hline 90 & 4.15 \\
\hline 100 & 4.76 \\
\hline
\end{tabular}




\section{Weight and Multiplier System}

The recommended approach for formalizing the process of transferring matrix diffusion data obtained from outside a given CAU and for determining the relative weight that should be assigned to the data is to use a weight and multiplier system. This approach is also recommended for datasets obtained within a CAU of interest because, even though all such data will come from a relevant geologic setting, some CAU-specific datasets should rightfully be assigned greater emphasis than others because of differences in measurement methods, data quality, and documentation quality.

In the weight and multiplier system approach, weights are determined for datasets based on the relevance of the data to the CAU, and then multipliers (factors that the total weight score is multiplied by to obtain an overall score) are assigned based on the measurement method, the quality of data reduction and analyses, and documentation quality. datasets with poor relevance, while they may still be transferred, are assigned lower weights so they ultimately "count less" in determining the final parameter distribution used in CAU-scale modeling. Likewise, datasets associated with measurement methods having large uncertainties or inappropriate scales, or datasets of low-quality or having poor documentation are assigned lower multipliers so that they count less in the final CAU-scale application.

The rationale for using both weights and multipliers is that the relevance and the data quality are two separate criteria that are largely independent. Weights are used for relevance because this is an overriding consideration in most cases. datasets obtained from the best possible measurement method and having high-quality analyses and documentation should nevertheless be rejected if the relevance is inappropriate. In a system based entirely on weights, such datasets could potentially still be assigned a relatively high weight score even though they are not appropriate for the CAU of interest. Multipliers are assigned after weights are determined so that appropriate credit can be taken for the measurement method and data/documentation quality. Although datasets will seldom be rejected outright at this stage, very low multipliers can be assigned because of a highly uncertain measurement method or very poor documentation. The weights and multipliers have relative scales rather than absolute scales. For instance, if there are only two datasets being considered, then multipliers of 1.0 and 0.5 for these sets will have the same effect as multipliers of 0.2 and 0.1 , respectively (i.e., the first dataset has a multiplier twice that of the second set in both cases).

This approach of "graded" transferability recognizes that most transfer decisions will not be a simple "yes or no", but rather there will be varying degrees of relevance of datasets to a given $\mathrm{CAU}$, as well as varying degrees of measurement 
and documentation quality. It also recognizes that, while the goal is to make objective decisions regarding data transferability, there are almost always subjective elements involved in these decisions.

Sections 3.2 through 3.4 provide guidance for determining the relevance of datasets to NTS CAUs. Sections 3.5 through 3.7 provide guidance for assessing measurement methods and for determining data reduction and documentation quality. The following two sections provide guidance for stepping through these considerations to assign weights and multipliers, respectively, to datasets.

\subsection{Weights (Data Relevance)}

The following guidance is provided for assigning weights, which are a measure of relevance, to datasets. Note that a weight score of zero essentially implies rejection of the data being considered for transfer.

\section{Matrix Porosity and Permeability (for $D_{m}$ Values)}

Weight factors for $D_{\mathrm{m}}$ values obtained from outside of NTS CAUs should be highly correlated with the "similarity" of matrix porosity and log matrix permeability at the two different locations. Of these two factors, log permeability should be given more emphasis than porosity. If the measured permeability of the matrices agrees within an order of magnitude ( $1 \log$ unit) at the two locations, an initial weight factor of 1 is appropriate. If permeabilities differ by 1 to 2 orders of magnitude, then a weight factor of 0.6 is assigned. For differences of 2 to 3 orders of magnitude, a weight factor of 0.3 is appropriate; and a weight factor of zero is assigned if permeability differences exceed 3 orders of magnitude. The weight factors are then further adjusted based on differences in matrix porosity. For matrix porosities that differ by less than 25 percent of the larger value, the initial weight factor determined from permeability differences is multiplied by 1 . When porosities differ by 25 to 90 percent of the larger value, the initial weight factor is multiplied by 0.7 . When porosities differ by more than an order of magnitude (greater than 90 percent of the larger value), the initial weight factor is multiplied by 0.4 . Thus, the overall weight factor is more heavily influenced by log permeability than porosity, although porosity still plays a role in determining the factor.

Note: If matrix diffusion coefficient data from outside of NTS CAUs are paired with matrix porosity and permeability data, then these data can be added to the overall dataset used in the multiple linear regression to obtain equation (B-17) regardless of what the matrix porosity and permeability values are. The regression equation can then be revised using these additional data. In this case, no data transfer considerations apply because the outside data are not used directly. Rather, they are included in the development of a new predictive regression equation that can be used when matrix porosity and permeability data are available for a given hydrostratigraphic unit for which no diffusion coefficient measurements are available. 


\section{Geologic Setting (for $D_{m}$ Values)}

For $D_{\mathrm{m}}$ values, the geologic setting should not be used as a transferability factor unless there are no matrix porosity and permeability data available (note that porosity data should be available because porosities are generally necessary to obtain diffusion coefficient estimates). If matrix porosity and permeability data are both not available, then there is no choice but to rely on similarity of geologic setting to determine a relevance weight factor. Similarities and differences in geologic setting (including rock type, deposition history, geochemical/hydrothermal alteration, structural setting, and mechanical alteration) should be evaluated and weights assigned using a sliding scale of 0 to 0.7 , with 0.7 indicating a very similar setting. The scale does not go to 1.0 because similarity of geologic setting is a more arbitrary measure of similarity for the purposes of transferring $D_{\mathrm{m}}$ values than comparing porosity and permeability data. Further guidance on how to assign sub-weights within the 0 -to- 0.7 sliding scale is not provided because of the multiplicity and complexity of considerations involved, as well as the desire to give the analyst flexibility for a wide range of potential situations. However, the burden is on the analyst to document the rationale for the assigned weight (Section 5.0). The analyst is encouraged to use the formal statistical methods of addressing similarity discussed in Section 3.1 of the Matrix Porosity Attachment A of this document to help determine and document a score.

\section{Geologic Setting (for $L$ and b Values)}

Geologic setting plays a much more prominent role in determining the transferability of $L$ and $b$ data from one location to another than $D_{\mathrm{m}}$ data. In this case, relevance weights should be assigned using a sliding scale of 0 to 1 , with 1 indicating a very similar setting. However, similarity of geologic setting should be evaluated differently for $L$ and $b$ data than for $D_{\mathrm{m}}$ data. Whereas matrix porosity and permeability are the overriding considerations for $D_{\mathrm{m}}$ data, rock hardness and structural setting will be more important for $L$ and $b$ data. Thus, geologic factors that may lead an analyst to conclude that two settings are quite similar for transferability of $D_{\mathrm{m}}$ data, may lead the same analyst to conclude that the settings are quite different for transfer of $L$ and $b$ data. Again, the analyst is encouraged to use the formal statistical methods of addressing similarity discussed in Section 3.1 to help determine and document a score.

Note: If CAU-scale models are developed that include domains of different rock types, deposition/alteration histories, and/or structural settings, individual datasets can be considered for each model domain and weights can be assigned on a case-by-case basis for each domain. For instance, given the concept of multiple HSUs in flow and transport models, a particular dataset could be "transferred" to different HSUs, with a different weight assigned to each HSU. This same approach can be extended to situations were HSUs contain multiple rock types or multiple deposition/alteration histories; the data could be transferred with different weights assigned to different portions of the HSU. Also, if different structural features are distinguished in model domains (e.g., faulted and unfaulted regions), datasets could be weighted differently for these different regions. 


\subsection{Multipliers (Measurement Method, Quality of Analyses and Documentation)}

Note that the overall multiplier for a dataset is the product of the multipliers for each of the criteria listed below.

\section{Measurement Method}

Measurement method considerations include both the quality/ uncertainty of the method and the scale of the measurement. General guidelines on relative rankings of measurement methods were discussed in Section 3.5. The numerical values listed below are intended for guidance only, but their values are chosen to reflect the relative emphasis that should be placed on different types of measurements for estimating matrix diffusion parameters for CAU-scale modeling (given current measurement technologies). Different methods and multipliers are listed separately for different matrix diffusion parameters. Higher multipliers reflect what is considered to be a "better" measurement. Any method not listed below should be considered on a case-by-case basis. An example of such a method is the analysis of geochemical and isotopic groundwater data discussed in Section 2.2.3, which is largely undemonstrated and for which the multiplier will depend strongly on the quality and extensiveness of the data and the sophistication of the analyses employed.

- $D_{\mathrm{m}}$ values:

- Laboratory methods that involve measuring diffusion through or into a rock matrix based on monitoring solute concentration increases or decreases on either side of the sample (e.g., diffusion cell experiments). -1.0 .

- Diffusion cell experiments in which both concentration increases and decreases are measured (on both sides of a sample) - 1.3.

- Laboratory methods based on conductivity measurements -0.5 .

- X-ray or neutron imaging measurements -1.0 .

- Multiplier for size of sample - 0.6 to 1.4, with larger multipliers assigned to larger samples.

- Multiplier for including a fracture surface in the sample - 1.2.

- $\quad M_{D}=\frac{\phi}{b \eta} \sqrt{D_{\mathrm{m}} \mathrm{R}_{\mathrm{m}}}$ values:

- Laboratory fracture transport experiments with multiple tracers - 0.3.

- Single-well field tracer transport test with multiple tracers - 0.8.

- Cross-hole field tracer transport test with multiple tracers - 1.2.

- Any laboratory or field tracer test with only a single nonsorbing tracer -0.1 . 
- Multiplier for tracer residence time in a field test -0.6 to 1.4 , with larger multipliers for longer-duration tests.

- Multiplier for length scale of a field test -0.8 to 1.2 , with larger multipliers for larger-scale tests.

- $L$ values:

- Borehole flow surveys with corresponding televiewer or television $\log \mathrm{s}-1.0$.

- Borehole flow surveys alone -0.7 .

- Estimates based on fracture density or fracture mapping - 0.1.

- Field tracer tests with multiple tracers in which the tracer responses deviate significantly from expected responses in a system with an "infinite" matrix -1.0 .

- $\quad b$ values:

- Estimates obtained from $M T C_{D}=\frac{\phi}{b \eta} \sqrt{D_{\mathrm{m}} \mathrm{R}_{\mathrm{m}}}$ values determined in field tracer tests, using independent estimates of $\phi$ and $D_{\mathrm{m}}$ to "back out" $b$ values from the lumped parameter -1.0 .

- Estimates based on hydraulic responses (i.e., hydraulic apertures) 0.2 .

- Estimates based on measurements of apertures in core samples or television $\log s-0.2$.

- $\mathrm{R}_{\mathrm{MD}}$ values:

- Estimates based on combining estimates of $L, b$, and $\phi$ into $1+\frac{\phi(L / 2)-b}{b}$ (assuming $\mathrm{R}_{\mathrm{m}}, \mathrm{R}_{\mathrm{f}}$, and $\left.\eta=1\right)-1.0$ times the multipliers for the $L^{b}$ and $b$ estimates given above.

- Estimates based on estimates of $\phi$ and effective flow porosity from cross-hole tracer tests (using equation B-15) - 0.3.

- Estimates based on estimates of $\phi$ and effective flow porosity from cross-hole tracer tests with corrections made using estimates of $L$ derived from field tracer tests (using equation B-16) - 0.6.

\section{Data Reduction and Analysis Methods}

Data reduction and analysis methods are evaluated independently of the type of measurement being evaluated. However, the analyst must be familiar with state-of-the-art methods to make an evaluation. The following multipliers apply (a sliding scale can be employed to allow for a continuum of scores): 
- Current and widely-accepted data reduction and analysis method with careful attention to detail in solute analysis (tracer methods) and/or other experimental/measurement details -1.0 .

- Good method, but attention to detail is suspect -0.5 .

- Based on older or less widely-accepted methods, but attention to detail is $\operatorname{good}-0.5$.

- $\quad$ Both methods and attention to detail are suspect -0.2 .

Note: The score for any poorly-analyzed dataset can be raised if the raw data are available and are re-analyzed using state-of-the-art methods. However, it is not possible to raise a score above 0.5 for datasets with poor attention to detail (unless other information can be used to effectively corroborate or validate the measurements).

\section{Quality of Documentation}

Documentation quality is also evaluated independently of the type of measurement being evaluated. The following multipliers apply (a sliding scale can be employed to allow for a continuum of scores):

- Good (thorough, easy to follow, traceable) - 1.0.

- Appearing in a reputable journal or peer-reviewed report, but otherwise lacking in thoroughness -0.6 .

- $\quad$ Poor -0.3

Note: It is often the case that poor documentation results in the inability to evaluate the data reduction and analysis method (including attention to detail) for a given dataset. Thus, poorly-documented estimates can, in effect, be doubly penalized as a result of both poor documentation scores and poor data reduction and analysis scores.

\subsection{Overall Scores}

The overall score for a given dataset is taken to be the weight score multiplied by the product of the multipliers. For example, if a dataset is assigned a weight score of 1.2 and then assigned multipliers of $0.8,1.0$, and 0.6 (for the three respective categories in Section 4.2), the overall score for the dataset is $1.2 \times 0.8 \times 1.0 \times 0.6=$ 0.576 . 


\section{Documentation Requirements}

For each dataset that is considered for use in CAU-scale flow and transport modeling, the following information should be documented:

- For rejected data, rationale for rejection (i.e., rationale for assigning a total weight score of zero).

- For accepted data, a summary of the data.

- The weight score.

- Multiplier scores for each category listed in Section 4.2.

- The rationales for the weight and multiplier scores.

- The overall score for the dataset.

A format for documenting this information is not specified, but each of the above elements should be addressed.

Documentation is very important because it is here that the weight and multiplier scores are justified. Assigning the weights and multipliers using the guidance and relative scales provided in Section 4.0 forces the analyst to consider the applicability and relative merits of each dataset. It is not so important that the analyst follow this guidance to the letter, but rather it is important that the analyst documents and justifies the assigned weights and multiplier scores. It is also important that the analyst is consistent in the assignment of weights and multipliers. Documentation ensures that the analyst's decisions are traceable and easy to review. 


\section{Use of Weights and Multipliers in Downstream Analyses}

The matrix diffusion data that are transferred to a given CAU (as well as CAU-specific data) can be used in many different ways in CAU-scale modeling. It is not the purpose of this attachment to recommend or exhaustively consider all the possible uses of the data or the ways in which the combined weight and multiplier scores should be incorporated into these uses. Rather, a specific and relatively common usage of data is considered, and the manner in which combined weight and multiplier scores are incorporated into other uses can be inferred from this discussion.

It is a common practice to compile CDFs of parameter values that are randomly sampled in Monte-Carlo fashion in large-scale models to simulate overall system performance. These CDFs are intended to capture the variability and uncertainty associated with parameter values within the model domain. The large-scale models are typically executed many times so that representative sampling of the component CDFs is accomplished. Finally, a CDF of the model output/predictions is constructed from the multiple model runs to allow an assessment of uncertainty in overall system performance.

The matrix diffusion coefficient is a good example of a parameter for which a CDF may be constructed and then randomly sampled. The suggested method of incorporating the combined weight and multiplier scores into a CDF is best illustrated by example. We assume that there are 4 datasets from which a total of 12 values of matrix diffusion coefficients have been obtained to represent a consolidated rock from a specific HSU within a given CAU. The four datasets are described as follows:

- A laboratory diffusion cell dataset with four separate measurements of matrix diffusion coefficients on different samples taken directly from the HSU. Solute concentration measurements were made only on the low concentration side of the diffusion cells. The solute in all cases was ${ }^{3} \mathrm{HHO}$. The measured diffusion coefficients were $2.3,6.2,1.1$, and $8.0 \mathrm{x}$ $10^{-6} \mathrm{~cm}^{2} / \mathrm{sec}$.

- A conductivity dataset with two separate measurements of matrix diffusion coefficients on samples taken from outside the CAU. Both samples had matrix porosities within 25 percent of the samples from the CAU, but the matrix permeabilities for the samples were 1 to 2 orders of magnitude lower than for the CAU samples. The anion used for the measurements was $\mathrm{Cl}^{-}$. The diffusion coefficients derived from the measurements were 1.2 and $1.8 \times 10^{-6} \mathrm{~cm}^{2} / \mathrm{sec}$. 
- A laboratory diffusion cell dataset with five separate measurements of matrix diffusion coefficients on different samples taken from outside the CAU. The first three samples have matrix porosities within 25 percent and permeabilities within 1 order of magnitude of the samples from the CAU. The last two samples have porosities 50 and 70 percent different and permeabilities between 1 and 2 orders of magnitude different than the samples from the CAU. Solute concentrations measurements were made on both sides of the diffusion cells. The solute in all cases was $\mathrm{Br}$. The diffusion coefficient measurements were $2.8,4.0$, and $5.7 \times 10^{-6} \mathrm{~cm}^{2} / \mathrm{sec}$ for the first three samples, and 0.9 and $1.3 \times 10^{-6} \mathrm{~cm}^{2} / \mathrm{sec}$ for the last two samples.

- A single measurement obtained from x-ray imaging using a sample from outside the CAU with a matrix porosity 30 percent different and a permeability within 1 order of magnitude of the samples from the CAU. $\mathrm{I}^{-}$was used for the measurements. The bulk diffusion coefficient obtained from the measurement was $1.5 \times 10^{-6} \mathrm{~cm}^{2} / \mathrm{sec}$.

Without going into the details of how and why, the combined weight and multiplier scores in Table 6-1 are assigned to the matrix diffusion data. Note that the scores for the HSU-specific diffusion cell measurements are assigned similar values because these measurements are assumed to be conducted as part of a single set of measurements (for this example) using the same analysis method. They are also assumed to be documented in a single report. The scores differ slightly because some of the diffusion cells are assumed to be thicker (longer diffusion distances) than others; measurements associated with thicker samples are considered more representative for CAU-scale modeling. Using a similar combined score, with only minor adjustments for scale, for multiple measurements generated within a single study is an acceptable practice when CAU-specific measurements are made in the study. When multiple non-CAU measurements are made within a given study, the weight values may vary for the different measurements because the relevance of the measurements to the CAU of interest may vary, but the multipliers should be the same (because the same methods and documentation presumably apply to all measurements). Similarly, when measurements on CAU-specific materials are obtained in different investigations and at different times, the weight scores (relevance) should be the same, but different multipliers can apply because of differences in methods or documentation. Finally, when measurements are obtained on non-CAU materials in different studies and at different times, both the weight scores and multipliers can be different.

Note that in Table 6-1, the highest scores are assigned to the non-CAU diffusion cell data because the samples had similar porosity and permeability to the CAU samples, and the measurement method was actually better than the CAU-specific measurements since it involved determining solute concentrations on both sides of the diffusion cell samples instead of just one side. Also, the non-CAU samples were similar in size to the CAU samples.

To construct a CDF from the data and combined scores of Table 6-1, it is necessary to sort the diffusion coefficient values in ascending order and then add 


\begin{tabular}{|c|c|c|}
\hline \multicolumn{3}{|c|}{$\begin{array}{c}\text { Table 6-1 } \\
\text { Combined Weight and Multiplier Scores } \\
\text { for Hypothetical Matrix Diffusion Coefficient Data }\end{array}$} \\
\hline \multirow{5}{*}{ CAU Diffusion Cells } & $\begin{array}{l}\text { Diffusion Coefficient } \\
\quad\left(\times 10^{6}, \mathrm{~cm}^{2} / \mathrm{sec}\right)\end{array}$ & Combined Score \\
\hline & 2.3 & 1.0 \\
\hline & 6.2 & 0.9 \\
\hline & 1.1 & 1.2 \\
\hline & 8.0 & 0.7 \\
\hline \multirow{2}{*}{ Non-CAU Conductivity } & 1.2 & 0.3 \\
\hline & 1.8 & 0.3 \\
\hline \multirow{5}{*}{ Non-CAU Diffusion Cells } & 2.8 & 1.3 \\
\hline & 4.0 & 1.3 \\
\hline & 5.7 & 1.0 \\
\hline & 0.9 & 0.9 \\
\hline & 1.3 & 0.7 \\
\hline $\begin{array}{l}\text { Non CAU X-Ray } \\
\text { measurements }\end{array}$ & 1.5 & 0.7 \\
\hline
\end{tabular}

the scores in cumulative fashion for each successive value. The cumulative score for each diffusion coefficient value is then divided by the sum of the scores for all diffusion coefficients to obtain an estimate of the cumulative probability for that diffusion coefficient measurement. Thus, the probability of each diffusion coefficient value is proportional to its combined score. The results of manipulating the data from Table 6-1 in the manner indicated above are shown in Table 6-2. Figure 6-1 shows the resulting CDF of matrix diffusion coefficient values.

For this example, the CDF does not appear to be heavily influenced by any single measurement or set of measurements. However, in some cases, a single "very good" measurement may have a combined score equal to or greater than the sum of scores of all the other available data. In such cases, there will be a large jump in the CDF at the value corresponding to the "very good" measurement.

This example also serves to illustrate the value of determining combined weight and multiplier scores for CAU-specific data in addition to non-CAU data. Even though the scores for CAU-specific data are not required to determine data transferability, they are essential for establishing a representative CDF.

If another method of data usage is employed in CAU-scale modeling, then the combined weight and multiplier scores should be used in some other manner to "weigh" the available datasets appropriately in the final analysis. The method(s) of translating combined scores into some measure of variability and/or uncertainty should be documented appropriately. 
Table 6-2

Results of Manipulation of Data in Table 6-1 to Obtain Cumulative Distribution Function of Matrix Diffusion Coefficients

\begin{tabular}{|c|c|c|c|}
\hline $\begin{array}{c}\text { Sorted Diffusion } \\
\text { Coefficient Value }\end{array}$ & $\begin{array}{c}\text { Combined } \\
\text { Score }\end{array}$ & Cumulative Score & $\begin{array}{c}\text { Cumulative } \\
\text { Probability }\end{array}$ \\
\hline \hline 0.9 & 0.9 & 0.9 & 0.087 \\
\hline 1.1 & 1.2 & 2.1 & 0.204 \\
\hline 1.2 & 0.3 & 2.4 & 0.233 \\
\hline 1.3 & 0.7 & 3.1 & 0.301 \\
\hline 1.5 & 0.7 & 3.8 & 0.369 \\
\hline 1.8 & 0.3 & 4.1 & 0.398 \\
\hline 2.3 & 1.0 & 5.1 & 0.495 \\
\hline 2.8 & 1.3 & 6.4 & 0.621 \\
\hline 4.0 & 1.3 & 7.7 & 0.748 \\
\hline 5.7 & 1.0 & 8.7 & 0.845 \\
\hline 6.2 & 0.9 & 9.6 & 0.932 \\
\hline 8.0 & 0.7 & 10.3 & 1.0 \\
\hline
\end{tabular}

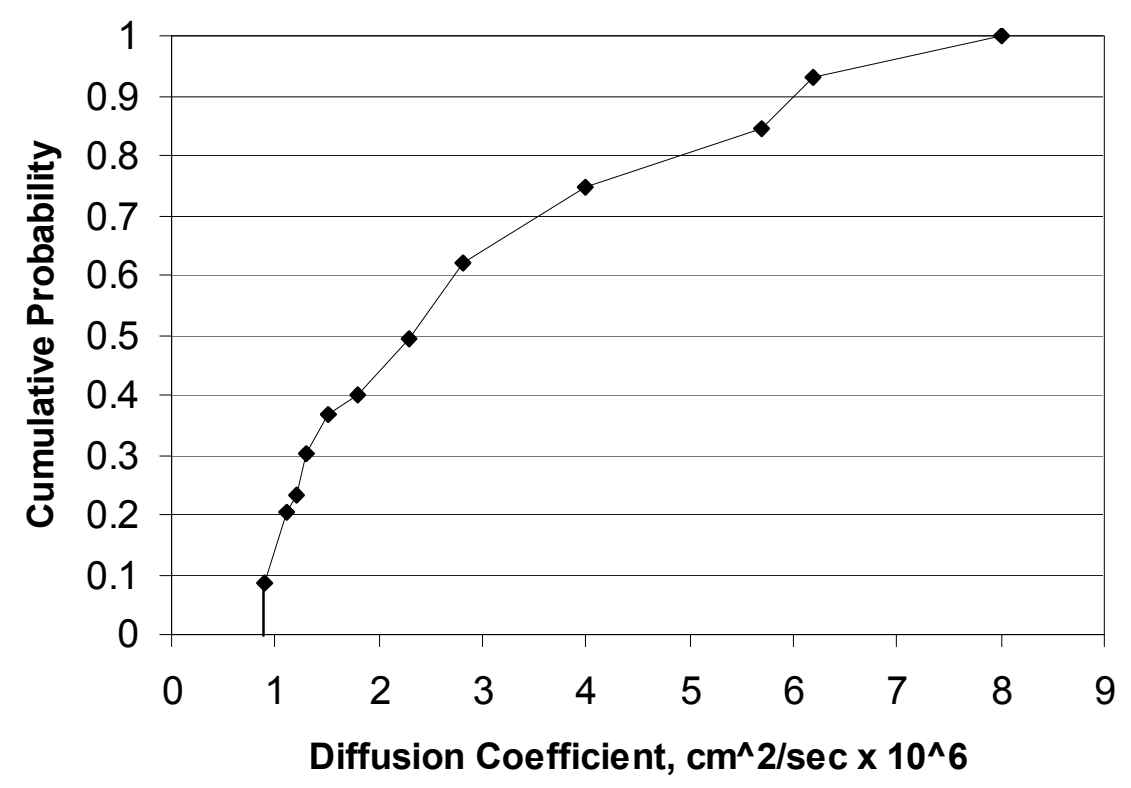

Figure 6-1

Cumulative Distribution Function Associated With the Matrix Diffusion Coefficient Data of Table 6-1 
Finally, there are many considerations of variability and uncertainty in parameter values that are not effectively captured by the weight and multiplier system and that go beyond the scope of this attachment. For instance, because weights and multipliers are assigned using relative scales, a CDF generated with only a few data points, data of questionable relevance, and poor-quality measurement methods might look exactly the same as one with many data points, CAU-specific data, and high-quality measurements. However, even though these CDFs may look essentially the same, the uncertainty should be far greater and the confidence far less in the former distribution than the latter one. In general, uncertainty in distributions of parameter values should be considered greater for (1) less total data points, (2) less CAU-specific data, and (3) smaller values of the sum of combined scores divided by the total number of data points. Methods of incorporating these measures of distribution uncertainty into a CAU-scale analysis go beyond the scope of this attachment. 
References

Becker, M.W. and A.M. Shapiro. 2000. "Tracer Transport in Fractured Crystalline Rock: Evidence of Nondiffusive Breakthrough Tailing." In Water Resour. Res., 36(7), 1677-1686.

Boving, T.B. and Grathwohl, P. 2001. "Tracer Diffusion Coefficients in Sedimentary Rocks: Correlation to Porosity and Hydraulic Conductivity." In J. Contaminant Hydrology, 53, 85-100.

Callahan, T.J., Reimus, P.W., Bowman, R.S., and Haga, M.J. 2000. "Using Multiple Experimental Methods to Determine Fracture/Matrix Interactions and Dispersion in Saturated Fractured Volcanic Tuff." In Water Resour. Res., 36(12), 3547-3558.

Carslaw, H.S. and J.C. Jaeger. 1959. Conduction of Heat in Solids. 2nd ed. Oxford: Oxford University Press.

Conca J.L., and J. Wright. 1990. "Diffusion Coefficients in Gravel Under Unsaturated Conditions." In Water Resour. Res., 26(5), 1055-1066.

Draper, N.R. and H. Smith. 1981. Applied Regression Analysis. $2^{\text {nd }}$ ed. New York, NY: John Wiley and Sons.

Durham W.B. and B.P. Bonner. 1994. "Self-Propping and Fluid-Flow in Slightly Offset Joints at High Effective Pressures." In J. Geophys. Res. - Solid Earth, 99(B5), 9391-9399.

Freeze, R.A. and J.A. Cherry. 1979. Groundwater. Englewood Cliffs, NJ: Prentice Hall.

Grisak, G.E. and J.F. Pickens. 1980. "Solute Transport Through Fractured Media. 1. The Effect of Matrix Diffusion." In Water Resources Research, 16, (4), 719-730.

Haggerty, R. and S. Gorelick. 1995. "Multiple-rate Mass Transfer for Modeling Diffusion and Surface Reactions in Media with Pore-scale Heterogeneity." In Water Resour. Res., 31(10), 2383-2400.

Kariuki, S. and H.D. Dewald. 1996. "Evaluation of Diffusion Coefficients of Metallic Ions in Aqueous Solutions." In Electroanalysis, 8(4), 307-313. 
Maloszewski, P. and A. Zuber. 1991. "Influence of Matrix Diffusion and Exchange Reactions on Radiocarbon Ages in Fissured Carbonate Aquifers." In Water Resour. Res., 27(8), 1937-1945.

Moench, A.F. 1995. "Convergent Radial Dispersion in a Double-Porosity Aquifer with Fracture Skin: Analytical Solution and Application to a Field Experiment in Fractured Chalk." In Water Resour. Res., 31(8), 1823-1835.

Neretnieks, I. 1980. "Diffusion in the Rock Matrix: An Important Factor in Radionuclide Migration?" In J. Geophys. Res., 85(B8), 4379-4397.

Newman, J.S. 1973. Electrochemical Systems. Englewood Cliffs, NJ: Prentice-Hall.

Reimus, P.W. 2003. "A Refined Approach to Estimating Effective Flow Porosity from Cross-Hole Tracer Tests in Fractured Media." In Proceedings of the $10^{\text {th }}$ International High-Level Radioactive Waste Management Conference, American Nuclear Society.

Reimus, P.W., S.D. Ware, F.C. Benedict, R.G. Warren, A.J. Humphrey, A.I. Adams, B. Wilson, and D. Gonzales. 2002. Diffusive and Advective Transport of ${ }^{3} \mathrm{H},{ }^{14} \mathrm{C}$, and ${ }^{99} \mathrm{Tc}$ in Saturated, Fractured Volcanic Rocks from Pahute Mesa, Nevada, LA-13891-MS. Los Alamos, NM: Los Alamos National Laboratory.

Reimus, P.W., and J.M. Haga. 1999. Analysis of Tracer Responses in the BULLION Forced-Gradient Experiment at Pahute Mesa, Nevada, LA-13615-MS. Los Alamos, NM: Los Alamos National Laboratory.

Rehfeldt, K., W. Drici, B. Lester, D. Sloop, J. Watrus, T. Beard, M.Sully, W. Fryer, and C. Benedict. 2004. Hydrologic Data for the Groundwater Flow and Contaminant Transport Model of Corrective Action Units 101 and 102: Central and Western Pahute Mesa, Nye County, Nevada, Rev. 0. Las Vegas, NV: Stoller Navarro Joint Venture.

Robinson, R.A., and R.H. Stokes. 1959. Electrolyte Solutions. $2^{\text {nd }}$ ed. London, England: Butterworths.

Sato, H., M. Yui, and H. Yoshikawa. 1996. "Ionic Diffusion Coefficient of $\mathrm{Cs}^{+}$, $\mathrm{Pb}^{2+}, \mathrm{Sm}^{3+}, \mathrm{Ni}^{2+}, \mathrm{SeO}_{4}{ }^{2-}$ and $\mathrm{TcO}_{4}^{-}$in Free Water Determined from Conductivity Measurements." In J. Nucl. Sci. Tech., 33(12), 950-955.

Shackleford, C.D. 1991. "Laboratory Diffusion Testing for Waste Disposal - A Review." In J. Contaminant Hydrology, 7, 177-217.

Triay, I.R., A. Meijer, J.L. Conca, K.S. Kung, R.S. Rundberg, E.A. Strietelmeier, C.D. Tait, D.L. Clark, M.P. Neu, and D.E. Hobart. 1997. Summary and Synthesis Report on Radionuclide Retardation for the Yucca Mountain Site Characterization Project, LA-13262-MS. Eckhardt, R.C., ed. Los Alamos, NM: Los Alamos National Laboratory. 
Weast, R.C., and M.J. Astle, eds. 1982. CRC Handbook of Chemistry and Physics, $62^{\text {nd }}$ Edition. Boca Raton, FL: CRC Press.

Wilson, J.E., L.B. Goodwin, and C.S. Lewis. "Deformation Bands in Non-Welded Ignimbrites: Petrophysical Controls on Fault-Zone Deformation and Evidence for Preferential Fluid Flow" Submitted to Geology. 


\section{Distribution}

R. M. Bangerter, Jr.

Environmental Restoration Division

U.S. Department of Energy

National Nuclear Security Administration

Nevada Site Office

P.O. Box 98518, M/S 505

Las Vegas, NV 89193-8518

W. R. Wilborn

Environmental Restoration Division

U.S. Department of Energy

National Nuclear Security Administration

Nevada Site Office

P.O. Box $98518, \mathrm{M} / \mathrm{S} 505$

Las Vegas, NV 89193-8518

J. P. McCord

Stoller-Navarro Joint Venture

7710 W. Cheyenne Ave. Bldg. 3

Las Vegas, NV 89129

B. J. Deshler

Stoller-Navarro Joint Venture

7710 W. Cheyenne Ave. Bldg. 3

Las Vegas, NV 89129

J. A. Wurtz

Stoller-Navarro Joint Venture

7710 W. Cheyenne Ave. Bldg. 3

Las Vegas, NV 89129

J. F. Pickens

Stoller-Navarro Joint Venture

7710 W. Cheyenne Ave. Bldg. 3

Las Vegas, NV 89129

G. J. Ruskauff

Stoller-Navarro Joint Venture

7710 W. Cheyenne Ave. Bldg. 3

Las Vegas, NV 89129

J. I. Daniels

Lawrence Livermore National Laboratory

P.O. Box 808, L-396

Livermore, CA 94551 
G. A. Pawloski

Lawrence Livermore National Laboratory

P.O. Box 808, L-231

Livermore, CA 94551

T. P. Rose

Lawrence Livermore National Laboratory

P.O. Box 808, L-221

Livermore, CA 94551

A. B. Kersting

Lawrence Livermore National Laboratory

P.O. Box 808, L-231

Livermore, CA 94551

R. M. Maxwell

Lawrence Livermore National Laboratory

P.O. Box 808, L-204

Livermore, CA 94551

A. Tompson

Lawrence Livermore National Laboratory

P.O. Box 808, L-204

Livermore, CA 94551

M. Zavarin

Lawrence Livermore National Laboratory

P.O. Box 808, L-231

Livermore, CA 94550

N. M. Becker

Los Alamos National Laboratory

P.O. Box 1663, M/S F665

Los Alamos, NM 87545

D. L. Finnegan

Los Alamos National Laboratory

P.O. Box 1663, M/S 462

Los Alamos, NM 87545

E. M. Kwicklis

Los Alamos National Laboratory

P.O. Box 1663, M/S T003

Los Alamos, NM 87545

W. L. Hawkins

Los Alamos National Laboratory

P.O. Box 1663, M/S 462

Los Alamos, NM 87545 
A. V. Wolfsberg

Los Alamos National Laboratory

P.O. Box 1663, M/S 649

Los Alamos, NM 87545

P. W. Reimus

Los Alamos National Laboratory

P.O. Box 1663, M/S J-534 CST-7

Los Alamos, NM 87545

H. J. Turin

Los Alamos National Laboratory

P.O. Box 1663, M/S J514

Los Alamos, NM 87545

P. K. Ortego

Bechtel Nevada

P.O. Box 98521, M/S NLV082

Las Vegas, NV 89193-8521

S. L. Drellack

Bechtel Nevada

P.O. Box 98521, M/S NLV082

Las Vegas, NV 89193-8521

V. Yucel

Bechtel Nevada

P.O. Box 98521, M/S NLV081

Las Vegas, NV 89193-8521

C. E. Russell

Desert Research Institute

755 E. Flamingo Road

Las Vegas, NV 89132-0040

G. M. Pohll

Desert Research Institute

755 E. Flamingo Road

Las Vegas, NV 89132-0040

R. L. Jacobson

Desert Research Institute 2215 Raggio Parkway

Reno, NV 89512

J. M. Thomas

Desert Research Institute 2215 Raggio Parkway

Reno, NV 89512 
R. K. Waddell

GeoTrans

363 Centennial Parkway, Suite 210

Louisville, CO 80030

R. J. Laczniak

U.S. Geological Survey

160 N. Stephanie St.

Henderson, NV 89074

B. K. Thompson

U.S. Geological Survey

160 N. Stephanie St.

Henderson, NV 89074

R. P. Graves

U.S. Geological Survey

160 N. Stephanie St.

Henderson, NV 89074

D. E. Prudic

U.S. Geological Survey

333 West Nye Lane, Room 203

Carson City, NV 89706

E. H. McKee

U.S. Geological Survey

345 Middlefield Road, MS 975

Menlo Park, CA 94025

Z. E. Peterman

U.S. Geological Survey

Denver Federal Center

21 Center Ave., MS 963

Lakewood, CO 80225

U.S. Department of Energy

National Nuclear Security Administration

Nevada Site Office Technical Library

P.O. Box $98518, \mathrm{M} / \mathrm{S} 505$

Las Vegas, NV 89193-8518

U.S. Department of Energy

1 (electronic copy)

Office of Scientific and Technical Information

P.O. Box 62

Oak Ridge, TN 37831-0062

Southern Nevada Public Reading Facility

c/o Nuclear Testing Archive

P.O. Box $98521, \mathrm{M} / \mathrm{S} 400$

Las Vegas, NV 89193-8521 
Manager, Northern Nevada FFACO

Public Reading Facility

c/o Nevada State Library \& Archives

Carson City, NV 89701-4285

Central Files

Stoller-Navarro Joint Venture

7710 W. Cheyenne Ave. M/S 439

Las Vegas, NV 89129 